Supporting Information

\title{
First-Principles and Microkinetic Study on Mechanism for Ammonia Synthesis Using Ru-Loaded Hydride \\ Catalyst
}

Takuya Nakao $^{1}$, Tomofumi Tada ${ }^{1 *}$ and Hideo Hosono ${ }^{{ }^{*}}$

${ }^{1}$ Materials Research Center for Element Strategy, Tokyo Institute of Technology, 4259

Nagatsuta, Midori-ku, Yokohama 226-8503, Japan

*e-mail: tada.t.ae@m.titech.ac.jp, hosono@mces.titech.ac.jp 


\section{Computational details}

It has been reported that nitrogen $\left(\mathrm{N}_{2}\right)$ molecule can be activated at the $\mathrm{B} 5$ site of $\mathrm{Ru}$ (Fig. S1a), a step site at Ru (0001), and a similar structure of the B5 site of Ru is required for reaction analysis using atomistic calculations. Foppa et al. reported a quasiB5 sites (Figure $\mathrm{S} 1 b)$ can appear on the surface of a $\mathrm{Ru}_{57}$ cluster $(\sim 1 \mathrm{~nm})$ and $\mathrm{Ru}_{323}$ cluster $(\sim 2 \mathrm{~nm})^{1}$, and we attempted to make a Ru-loaded $\mathrm{Ca}_{2} \mathrm{NH}$ (100) model for reaction analysis in which the quasi-B5 of Ru appears. We succeeded in making the quasi-B5 site shaped $\mathrm{Ru}_{5}$ cluster on $\mathrm{Ca}_{2} \mathrm{NH}$ by taking into account the fact that $\mathrm{Ru}$ and $\mathrm{N}$ shows a strong chemical bond. ${ }^{2,3}$ The details for the model construction are as follows; in order to keep the shape of the quasi-B5 site of $\mathrm{Ru}_{5}$ cluster, two calcium on the $\mathrm{Ca}_{2} \mathrm{NH}$ (100) surface were removed to create a void, and we deposited the $\mathrm{Ru}_{5}$ cluster onto the void as shown in Figure S1c-d, where the edge four Ru atoms are bonded with the neighboring nitrogen, and the shape of the $\mathrm{Ru}_{5}$ cluster is apparently kept even after the structural relaxation (Figure S1d). To keep the charge neutrality of the $\mathrm{Ca}_{2} \mathrm{NH}$ slab, four hydrogen atoms were removed from the inner layers of the $\mathrm{Ca}_{2} \mathrm{NH}$ slab, and the model corresponds to the stoichiometry model of $\mathrm{Ru}_{5} / \mathrm{Ca}_{2} \mathrm{NH}$ (100). For the electron injection into $\mathrm{Ru}_{5}$ cluster, we additionally introduce two hydrogen vacancies $\left(\mathrm{V}_{\mathrm{H}}\right)$ around the $\mathrm{Ru}_{5}$ cluster shown in Figure $\mathrm{S} 1 \mathrm{~d}$, because anionic electrons can be generated at the $\mathrm{V}_{\mathrm{H}} \mathrm{s}$. The vacancy model is abbreviated as $\mathrm{Ru}_{5} / \mathrm{Ca}_{2} \mathrm{NH}_{1-\mathrm{x}} \mathrm{e}_{\mathrm{x}}^{-}$ (100). We investigated the $\mathrm{N}_{2}$ cleavage mechanism with or without the electron injection by using these models.

a

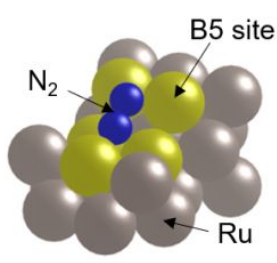

b

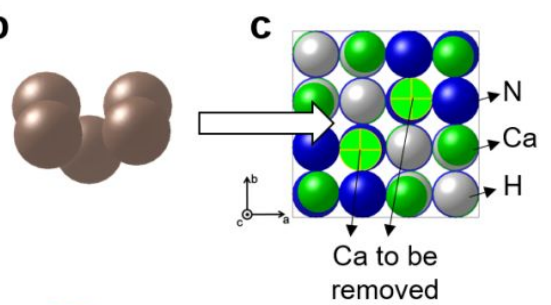

d

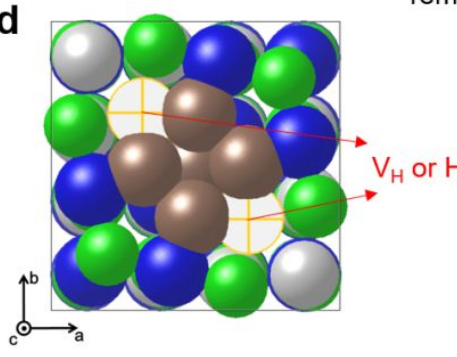

Figure S1. (a) Configuration of B5 site and $\mathrm{N}_{2}$ on $\mathrm{Ru}$ (0001) step. (b) Shape of the quasi-B5 site. (c) $\mathrm{Ca}_{2} \mathrm{NH}$ (100) surface. (d) Top view of $\mathrm{Ru}_{5}$-loaded $\mathrm{Ca}_{2} \mathrm{NH}$. 


\section{N-N bond length and activation energy for $N-N$ bond cleavage of nitrogen molecules adsorbed on different $R u$ clusters}

In this study, we first explored the active site for the $\mathrm{N}_{2}$ cleavage on $\mathrm{Ru}$ cluster which reproduces the activation energy for the $\mathrm{N}_{2}$ cleavage at the B5 site on Ru surface. The $\mathrm{N}-\mathrm{N}$ bond length of $\mathrm{N}_{2}$ adsorbed on the active site is a critical indicator for the activation energy of the N-N bond cleavage so that we explored the active sites for the $\mathrm{N}-\mathrm{N}$ bond cleavage where the N-N bond length is almost the same to that of the B5 site on $\mathrm{Ru}$ surface (1.32 $\AA$ ). Table $\mathrm{S} 1$ shows the $\mathrm{N}-\mathrm{N}$ bond lengths and the activation energies for $\mathrm{N}-\mathrm{N}$ bond cleavage of $\mathrm{N}_{2}$ adsorbed on different $\mathrm{Ru}$ clusters which we explored in this study. The $\mathrm{N}_{2}$ adsorbed at the quasi-B5 site on $\mathrm{Ru}_{5}$ cluster only has a little larger $\mathrm{N}-\mathrm{N}$ bond length than that at the B5-site on Ru surface. Therefore, we chose the $\mathrm{Ru}_{5}$ cluster with the quasi-B5 site for the further calculations about $\mathrm{N}_{2}$ dissociation and $\mathrm{NH}_{x}$ formation reactions. 
Table S1. N-N bond lengths and activation energies for N-N bond cleavage of nitrogen molecules adsorbed on different Ru clusters

\begin{tabular}{|c|c|c|c|c|c|}
\hline & Adsorption site & $N\left(\mathrm{~V}_{\mathrm{H}}\right)^{a}$ & $\mathrm{CN}^{b}$ & $\begin{array}{c}d(\mathrm{~N}-\mathrm{N})^{c} \\
{[\AA]}\end{array}$ & $\begin{array}{c}E_{\mathrm{a}}^{\mathbf{d}} \\
{[\mathrm{eV}]}\end{array}$ \\
\hline $\begin{array}{c}\text { Slab } \\
\text { (Ru only) }\end{array}$ & B5 site & - & 5 & 1.32 & 0.58 \\
\hline $\mathrm{Ru}_{4}$ & 4fold-hollow & 0 & 4 & 1.27 & - \\
\hline $\mathrm{Ru}_{5}$ & quasi-B5 site & 0 & 4 & 1.34 & 0.51 \\
\hline $\mathrm{Ru}_{5}$ & quasi-B5 site & 2 & 4 & 1.38 & 0.27 \\
\hline $\mathrm{Ru}_{6}$ & bridge1 & 1 & 2 & 1.20 & - \\
\hline $\mathrm{Ru}_{6}$ & bridge 2 & 0 & 2 & 1.20 & - \\
\hline $\mathrm{Ru}_{6}$ & bridge2 & 1 & 2 & 1.18 & - \\
\hline $\mathrm{Ru}_{6}$ & bridge 3 & 0 & 2 & 1.20 & - \\
\hline $\mathrm{Ru}_{6}$ & bridge 3 & 1 & 2 & 1.19 & - \\
\hline $\mathrm{Ru}_{6}$ & bridge3 & 2 & 2 & 1.17 & - \\
\hline $\mathrm{Ru}_{6}$ & bridge4 & 0 & 2 & 1.21 & 1.06 \\
\hline $\mathrm{Ru}_{6}$ & bridge 5 & 1 & 2 & 1.25 & 1.04 \\
\hline $\mathrm{Ru}_{6}$ & bridge6 & 1 & 2 & 1.23 & 1.54 \\
\hline $\mathrm{Ru}_{6}$ & 4fold-hollow & 0 & 4 & 1.24 & 0.75 \\
\hline $\mathrm{Ru}_{6}$ & 4fold-hollow & 2 & 4 & 1.28 & 0.61 \\
\hline $\mathrm{Ru}_{7}$ & 4fold-hollow1 & 0 & 4 & 1.29 & - \\
\hline $\mathrm{Ru}_{7}$ & 4 fold-hollow1 & 1 & 4 & 1.29 & - \\
\hline $\mathrm{Ru}_{20}$ & bridge & 0 & 5 & 1.28 & - \\
\hline $\mathrm{Ru}_{35}$ & 4fold-hollow & 2 & 4 & 1.30 & - \\
\hline $\mathrm{Ru}_{36}$ & 4fold-hollow & 2 & 4 & 1.27 & - \\
\hline $\mathrm{Ru}_{39}$ & 4fold-hollow & 2 & 4 & 1.26 & - \\
\hline
\end{tabular}

${ }^{a}$ Number of $\mathrm{V}_{\mathrm{H}}$ around Ru cluster. ${ }^{b}$ Coordination number of Ru-N. ${ }^{c} \mathrm{~N}-\mathrm{N}$ bond length of adsorbed $\mathrm{N}_{2} \cdot{ }^{d}$ Activation energy for $\mathrm{N}_{2}$ cleavage. 


\section{MD simulation for $\mathrm{Ru}_{5} / \mathrm{Ca}_{2} \mathrm{NH}_{1-x} \mathrm{e}^{-}$}

To investigate the stability of $\mathrm{Ru}_{5}$ cluster on $\mathrm{Ca}_{2} \mathrm{NH}$, we conducted the MD simulation for $\mathrm{Ru}_{5} / \mathrm{Ca}_{2} \mathrm{NH}$ at $400^{\circ} \mathrm{C}$ for $1 \mathrm{ps}$. Figure $\mathrm{S} 2$ shows the total energy and the distance of $\mathrm{Ru}-\mathrm{Ru}(d(\mathrm{Ru}-\mathrm{Ru}))$ of $\mathrm{Ru}_{5} / \mathrm{Ca}_{2} \mathrm{NH}$ model as a function of time. Although detailed structural changes such as $\mathrm{Ru}-\mathrm{Ru}$ distances happen during the MD simulation (the $d(\mathrm{Ru}-\mathrm{Ru})$ changes between $2.2 \AA$ to $3.1 \AA$ ), the overall shape of the quasi-B5 site on $\mathrm{Ru}_{5}$ cluster is kept (see Figure $\mathrm{S} 2 \mathrm{~b}$-e in SI), which indicates that the quasi-B5 site on $\mathrm{Ru}_{5}$ cluster is stable at $300-400^{\circ} \mathrm{C}$ (experimental temperature).
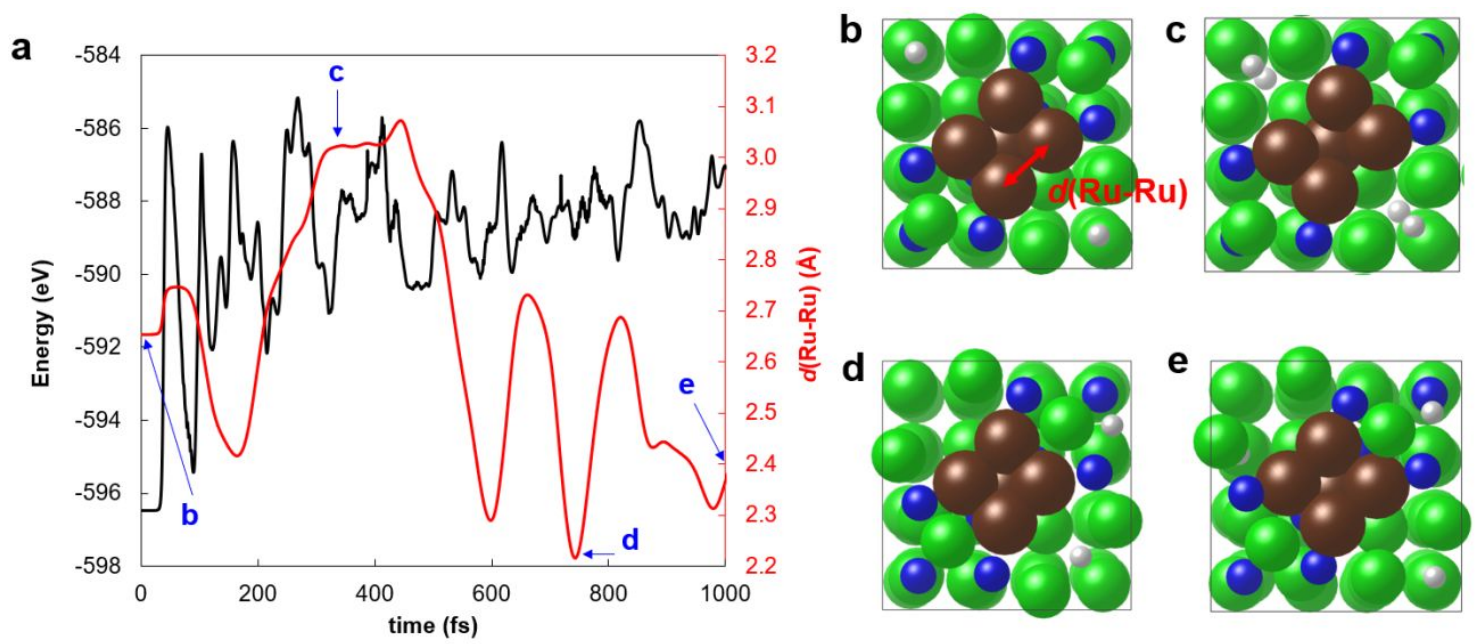

Figure S2. The total energies (black) of $\mathrm{Ru}_{5} / \mathrm{Ca}_{2} \mathrm{NH}$ and the distances (red) of $\mathrm{Ru}-\mathrm{Ru}$ $(d(\mathrm{Ru}-\mathrm{Ru}))$ of $\mathrm{Ru}_{5} / \mathrm{Ca}_{2} \mathrm{NH}$ model during the $\mathrm{MD}$ simulation at $400^{\circ} \mathrm{C}$. 


\section{Potential energy diagram of $\mathrm{NH}$ formation on $\mathrm{Ru}_{5} / \mathrm{Ca}_{2} \mathrm{NH}_{1-x} \mathrm{e}^{-}$}

The potential energy diagram of Langmuir-Hinshelwood (LH)-type NH formation on $\mathrm{Ru}_{5} / \mathrm{Ca}_{2} \mathrm{NH}_{1-x} \mathrm{e}_{x}^{-}$, and its configurations of the initial state (IS), transition state (TS) and final state (FS) are given in Figure S3. The activation energy of LH-type NH formation is $0.99 \mathrm{eV}$, which is higher than that of Mars-van Krevelen (MvK)-type NH formation $(0.74 \mathrm{eV})$.

a

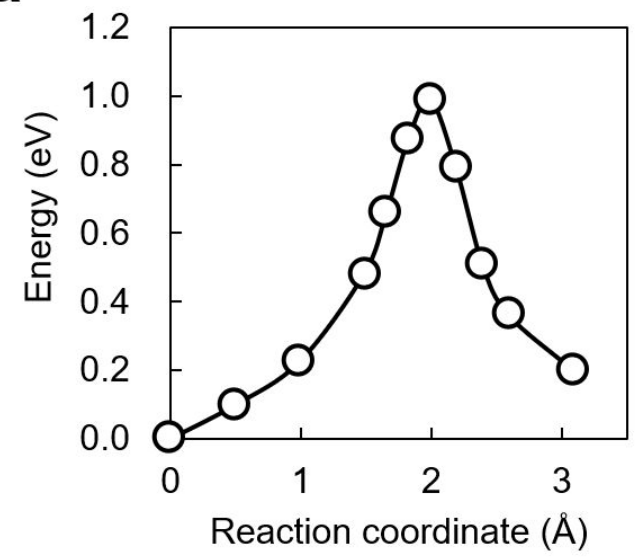

b

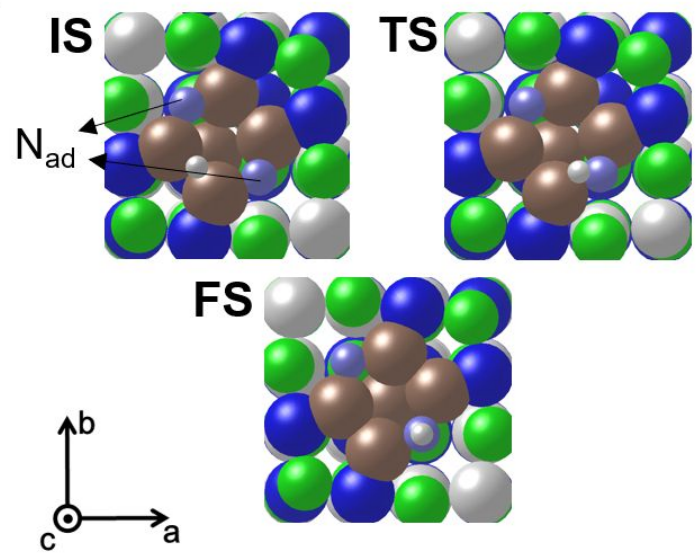

Figure S3. (a) Potential energy diagram for $\mathrm{NH}$ formation on $\mathrm{Ru}_{5} / \mathrm{Ca}_{2} \mathrm{NH}_{1-x} \mathrm{e}^{-}{ }_{x}$. (b) Configurations of the IS, TS, and FS of the LH-type NH formation. 


\section{COHP of Ru-H and adsorption energy of hydrogen in the NH formation}

The crystal orbital Hamiltonian population (COHP) of $\mathrm{Ru}-\mathrm{H}$ and the adsorption energies of the $\mathrm{H}$ atom in the initial state of the LH-type and Mars-van Krevelen (MvK)-type $\mathrm{NH}$ formation are given in Figure $\mathrm{S} 4$ and Table $\mathrm{S} 2 . E_{\text {ads }}(\mathrm{H})$ is defined in this study as $E_{\text {ads }}(\mathrm{H})=E\left(\mathrm{Ru}_{5}-\mathrm{H} / \mathrm{Ca}_{2} \mathrm{NH}_{1-x} \mathrm{e}_{x}^{-}\right)-1 / 2 E\left(\mathrm{H}_{2}\right)-E\left(\mathrm{Ru}_{5} / \mathrm{Ca}_{2} \mathrm{NH}_{1-x} \mathrm{e}_{x}^{-}\right)$, where $E(\mathrm{~A})$ indicates the total energy of system A. Note that according to the definition of $E_{\text {ads }}(\mathrm{H})$ in this study, the large negative value of $E_{\text {ads }}(\mathrm{H})$ indicates a strong adsorption of $\mathrm{H}$ on the support. The integrated COHP (ICOHP) obtained by integrating the COHP up to the Fermi level.

a

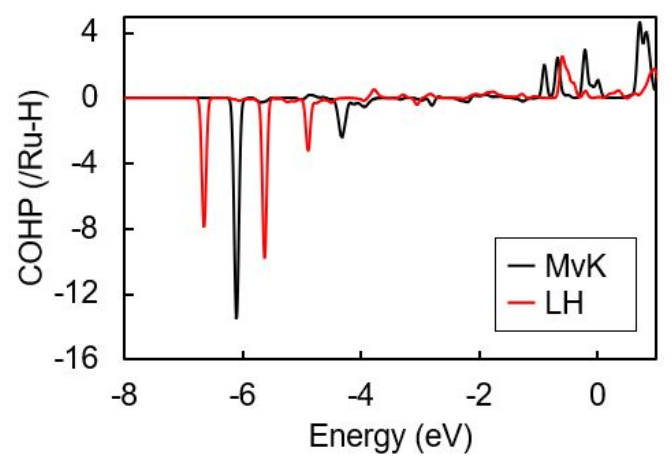

b
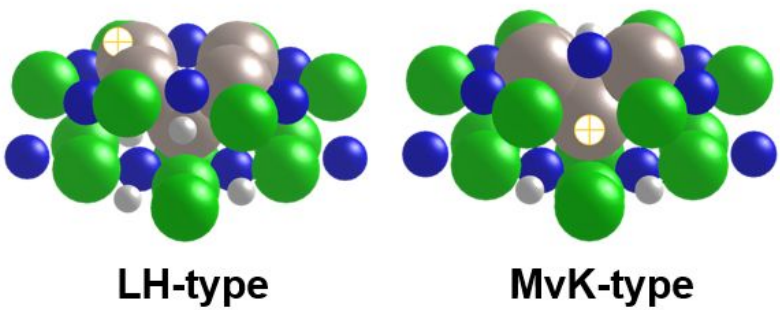

Figure S4. (a) COHP between Ru-H in the initial state of Langmuir-Hinshelwood-type and Mars-van Krevelen-type NH formation. (b) Configurations of the IS of the LH-type and MvK-type NH formation. The atom indicated with cross symbols is the hydrogen $(\mathrm{H})$ which forms $\mathrm{NH}$ by the reaction with the dissociated nitrogen atom.

Table S2. Adsorption energies of the $\mathrm{H}$ atom and the integrated COHP in the IS of LHtype and MvK-type $\mathrm{NH}$ formation

\begin{tabular}{ccc}
\hline & $\begin{array}{c}E_{\text {ads }}(\mathrm{H}) \\
{[\mathrm{eV}]}\end{array}$ & $\begin{array}{c}\text { ICOHP } \\
{[\mathrm{eV}]}\end{array}$ \\
\hline LH-type & -0.28 & -1.29 \\
MvK-type & -0.19 & -1.12 \\
\hline
\end{tabular}




\section{NH formation from a dissociated $\mathrm{N}$ adatom on $\mathrm{Ru}$ and surface $\mathrm{H}^{-}$on $\mathrm{Ca}_{2} \mathbf{N H}$}

The potential energy diagram of $\mathrm{NH}$ formation initiated by a dissociated $\mathrm{N}$ adatom on $\mathrm{Ru}$ and surface $\mathrm{H}^{-}$on $\mathrm{Ca}_{2} \mathrm{NH}$, and its configurations of the initial state (IS), the transition state (TS) and the final state (FS) are shown in Figure S5. The activation energy of the $\mathrm{NH}$ formation on $\mathrm{Ca}_{2} \mathrm{NH}$ is $4.09 \mathrm{eV}$, which is much higher than that of Mars-van Krevelen (MvK)-type NH formation (0.74 eV).
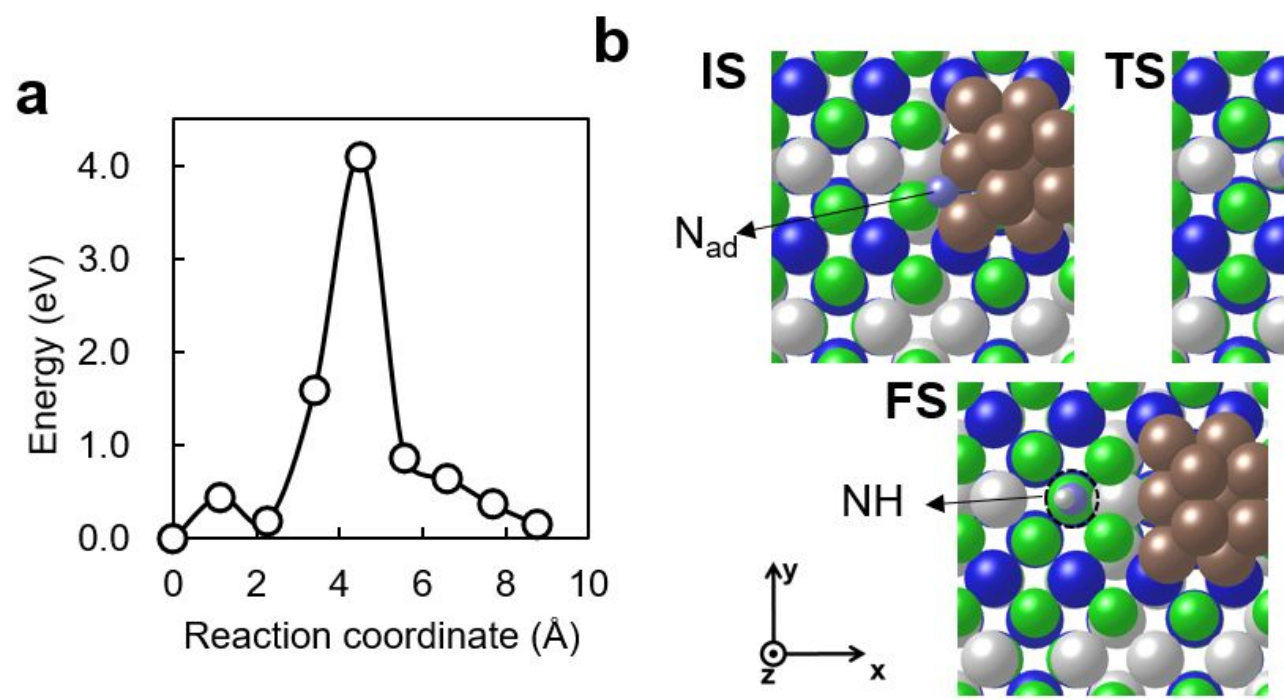

Figure S5. (a) Potential energy diagram for $\mathrm{NH}$ formation from dissociated $\mathrm{N}$ adatom on $\mathrm{Ru}$ and surface $\mathrm{H}^{-}$on $\mathrm{Ca}_{2} \mathrm{NH}$. (b) Configurations of the IS, TS, and $\mathrm{FS}$ of the $\mathrm{NH}$ formation. 


\section{Migration of lattice nitrogen of $\mathrm{Ca}_{2} \mathrm{NH}$ to $\mathrm{Ru}$ surface}

The potential energy profile of the migration of the lattice nitrogen atom of $\mathrm{Ca}_{2} \mathrm{NH}$ to $\mathrm{Ru}$ surface and its configurations of the initial state (IS), the transition state (TS) and the final state (FS) are shown in Figure S6. The activation energy of the $\mathrm{N}$ migration to Ru surface is $1.44 \mathrm{eV}$, which is much higher than the migration of lattice hydrogen of $\mathrm{Ca}_{2} \mathrm{NH}$ to $\mathrm{Ru}, 0.44 \mathrm{eV}$. The large activation energy of the migration of lattice $\mathrm{N}$ of $\mathrm{Ca}_{2} \mathrm{NH}$ is explained as follows. $\mathrm{Ru}$ and surface $\mathrm{N}$ of $\mathrm{Ca}_{2} \mathrm{NH}$ form a strong covalent bond ${ }^{2,3}$. A large activation energy is considered to be required to break the strong Ru-N covalent bond for the migration of $\mathrm{N}$ atom to $\mathrm{Ru}$.

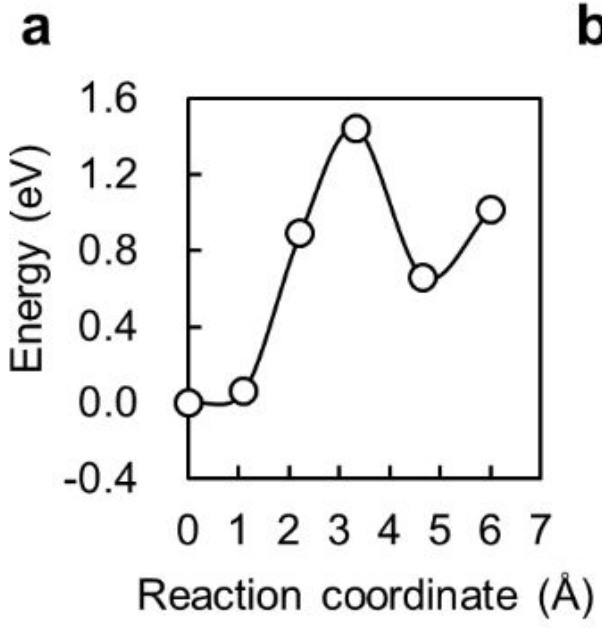

b

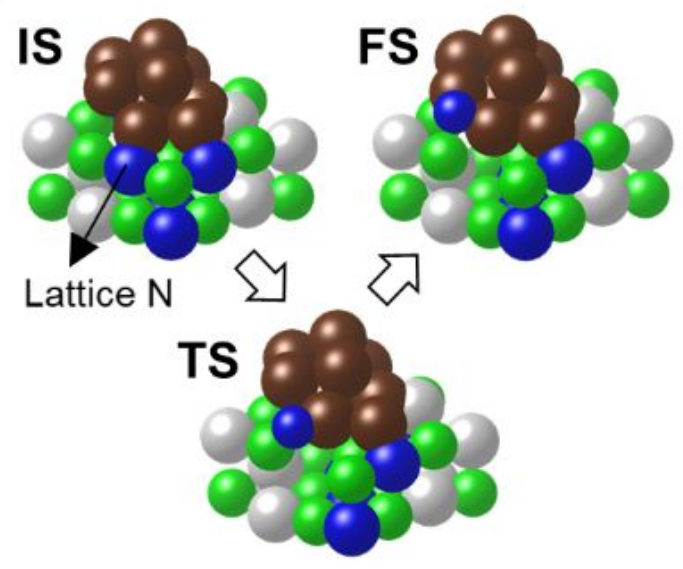

Figure S6. (a) Potential energy profile and (b) configurations of the IS, TS, and FS for the migration of lattice nitrogen of $\mathrm{Ca}_{2} \mathrm{NH}$ to $\mathrm{Ru}$. 


\section{Vibrational frequencies}

Table S3 lists the calculated vibrational frequencies for all the intermediates. The frequencies used to determine the vibrational partition functions, from which we can calculate entropy contributions.

Table S3. Vibrational frequencies for all the intermediates.

\begin{tabular}{lll}
\hline Species & State & Frequencies $[\mathrm{meV}]$ \\
\hline $\mathrm{N}_{2}{ }^{*}$ & IS & $96,74,65,61,44,36$ \\
$\mathrm{~N}_{2}{ }^{*}(\mathrm{TS})$ & TS & $80,73,60,47,35$ \\
$2 \mathrm{~N}^{*}$ & FS & $76,76,66,42,28,16$ \\
$\mathrm{~N}^{*}$ and $\mathrm{H}^{*}$ & IS & $187,122,81,59,39,34$ \\
$\mathrm{~N}-\mathrm{H}^{*}(\mathrm{TS})$ & $\mathrm{TS}$ & $137,73,56,44,30$ \\
$\mathrm{NH}^{*}$ & FS & $408,107,81,62,52,28$ \\
$\mathrm{NH}^{*}$ and $\mathrm{H}^{*}$ & IS & $416,223,120,109,81,69,57,53,29$ \\
$\mathrm{NH}^{-} \mathrm{H}^{*}(\mathrm{TS})$ & $\mathrm{TS}$ & $410,136,99,67,63,52,44,24$ \\
$\mathrm{NH}_{2}{ }^{*}$ & FS & $425,413,183,95,90,52,47,43,22$ \\
$\mathrm{NH}_{2}{ }^{*}$ and $\mathrm{H}^{*}$ & IS & $434,422,186,154,127,91,87,81,63,55,39,20$ \\
$\mathrm{NH}_{2}-\mathrm{H}(\mathrm{TS})$ & $\mathrm{TS}$ & $434,422,221,186,134,115,71,61,45,29,18$ \\
$\mathrm{NH}_{3}{ }^{*}$ & FS & $432,425,408,196,195,141,74,73,44,17,16,13$ \\
$\mathrm{H}_{\mathrm{s}}$ & IS & $152,79,59$ \\
$\mathrm{H}_{\mathrm{s}}(\mathrm{TS})$ & TS & 198,28 \\
$\mathrm{H}^{*}$ & FS & $230,45,38$ \\
\hline
\end{tabular}




\section{Rate expressions for ammonia synthesis on $\mathrm{Ru} / \mathrm{Ca}_{2} \mathrm{NH}_{1-x} \mathrm{e}^{-}$}

In order to construct microkinetic modeling for ammonia synthesis over $\mathrm{Ru} / \mathrm{Ca}_{2} \mathrm{NH}$, we calculated the rate constant of each elementary step as follows.

The rate constant, $k$, is expressed as: $\quad k=A \exp \left(-\frac{E_{\mathrm{a}}}{k_{\mathrm{B}} T}\right) \#(\mathrm{~S} 1)$

where $A$ is the pre-exponential factor, and $E_{\mathrm{a}}$ is the activation energy of the reaction.

According to the transition state theory, the rate constant is also expressed by using the partition functions of the initial and transition states and the activation energy:

$$
k=\frac{k_{\mathrm{B}} T q_{\mathrm{TS}}}{h q_{\mathrm{IS}}} \exp \left(-\frac{E_{\mathrm{a}}}{k_{\mathrm{B}} T}\right) \#(\mathrm{~S} 2)
$$

where $q_{\text {IS }}$ and $q_{\text {TS }}$ are the partition functions of the initial and transition states, respectively.

Comparing of Eq. $\mathrm{S} 1$ and Eq. $\mathrm{S} 2$, the pre-exponential factor, $A$ is expressed as:

$$
A=\frac{k_{\mathrm{B}} T q_{\mathrm{TS}}}{h q_{\mathrm{IS}}} \#(\mathrm{~S} 3)
$$

The partition function, $q$ is expressed as:

$$
q=q_{\text {trans }} q_{\text {rot }} q_{\text {vib }} q_{\text {elec }} \#(\mathrm{~S} 4)
$$

where $q_{\text {trans }}$ is the translational partition function, $q_{\text {rot }}$ is the rotational partition function, $q_{\mathrm{vib}}$ is the vibrational partition function, and $q_{\mathrm{elec}}$ is the electronic partition function. For gaseous $\mathrm{N}_{2}, \mathrm{H}_{2}$ and $\mathrm{NH}_{3}, q_{\text {elec }}=1$ (i.e., all the populations were assumed to be in the ground electronic level).

In the harmonic approximation, the contributions from the rotational and translational modes can be negligible. Therefore, the partition function of the transition state is written simply by the vibrational partition function as:

$$
q_{v i b}=\prod_{i} \frac{1}{1-\exp \left(-{ }^{\hbar} \omega_{i / k_{\mathrm{B}} T}\right)} \#(\mathrm{~S} 5)
$$

where $\omega_{\mathrm{i}}$ is the angular frequency of the $i$ 'th vibrational mode.

The partition function for the initial state contains vibrational, rotational and translational contributions from gaseous $\mathrm{N}_{2}, \mathrm{H}_{2}$, and $\mathrm{NH}_{3}$. The rotational partition function can be calculated as: $\quad q_{\text {rot }}=\frac{k_{\mathrm{B}} T}{\sigma h c B} \#(\mathrm{~S} 6)$ 


$$
q_{\text {rot }}=\frac{1}{\sigma}\left(\frac{k_{B} T}{h c}\right)^{3 / 2}\left(\frac{\pi}{A B C}\right)^{1 / 2} \#(\mathrm{~S} 7)
$$

where $\sigma$ is known as the rotational symmetry number, and $A, B$, and $C$ are the rotational constants of the molecules. Eq. S6 can be used for the linear molecule, and Eq. S7 can be used for the non-linear molecule.

The translational partition function is written as: $\quad q_{\text {trans }}=\frac{\left(2 \pi m k_{\mathrm{B}} T\right)^{3 / 2} k_{\mathrm{B}} T}{h^{3}} \#(\mathrm{~S} 8)$ where $m$ is the mass of the molecule, and $p_{\mathrm{X}}$ is the partial pressure of $\mathrm{X}$.

In this study, we used an effective partial pressure as the partial pressure in Eq. S8. An effective partial pressure, $p$ ' is expressed as:

$$
p^{\prime}=\frac{n_{\text {edge }}}{n_{\text {surface }}+n_{\mathrm{Ca}_{2} \mathrm{NH} \text { surface }}} p \#(\mathrm{~S} 9)
$$

where $n_{\text {edge }}$ and $n_{\text {surface }}$ is the number of the edge sites and the surface sites of the Ru particles on $\mathrm{Ca}_{2} \mathrm{NH}$, which are shown as Eq. S11 and $\mathrm{S} 12$. $n_{\mathrm{Ca}_{2} \mathrm{NH}}$ surface is the number of the atoms of $\mathrm{Ca}_{2} \mathrm{NH}(100)$, which is the most stable surface among the low-index surface. 


\section{Active sites for $\mathrm{NH}_{3}$ synthesis on $\mathrm{Ru} / \mathrm{Ca}_{2} \mathrm{NH}_{1-x} \mathrm{e}^{-}$}

Figure $\mathrm{S} 7$ shows the schematics of the reaction sites for $\mathrm{NH}_{3}$ synthesis using $\mathrm{Ru} / \mathrm{Ca}_{2} \mathrm{NH}_{1-x} \mathrm{e}_{x}^{-}$. Although there are several candidates for the active sites in $\mathrm{NH}_{3}$ synthesis on $\mathrm{Ru}$ particle as terrace, step and edge sites of $\mathrm{Ru}$, we adopted the edge site of $\mathrm{Ru}$ supported by the surface $\mathrm{H}^{-}$on $\mathrm{Ca}_{2} \mathrm{NH}$ as active sites, which are depicted with red dotted circles in Figure S7. The assumption is supported by the fact that the presence of the surface $\mathrm{H}^{-}$or $\mathrm{H}^{-}$-vacancy sites is required for $\mathrm{NH}_{3}$ synthesis on $\mathrm{Ru} / \mathrm{Ca}_{2} \mathrm{NH}_{1-x} \mathrm{e}^{-}$to avoid the hydrogen poisoning on $\mathrm{Ru}$, Ref 4 .

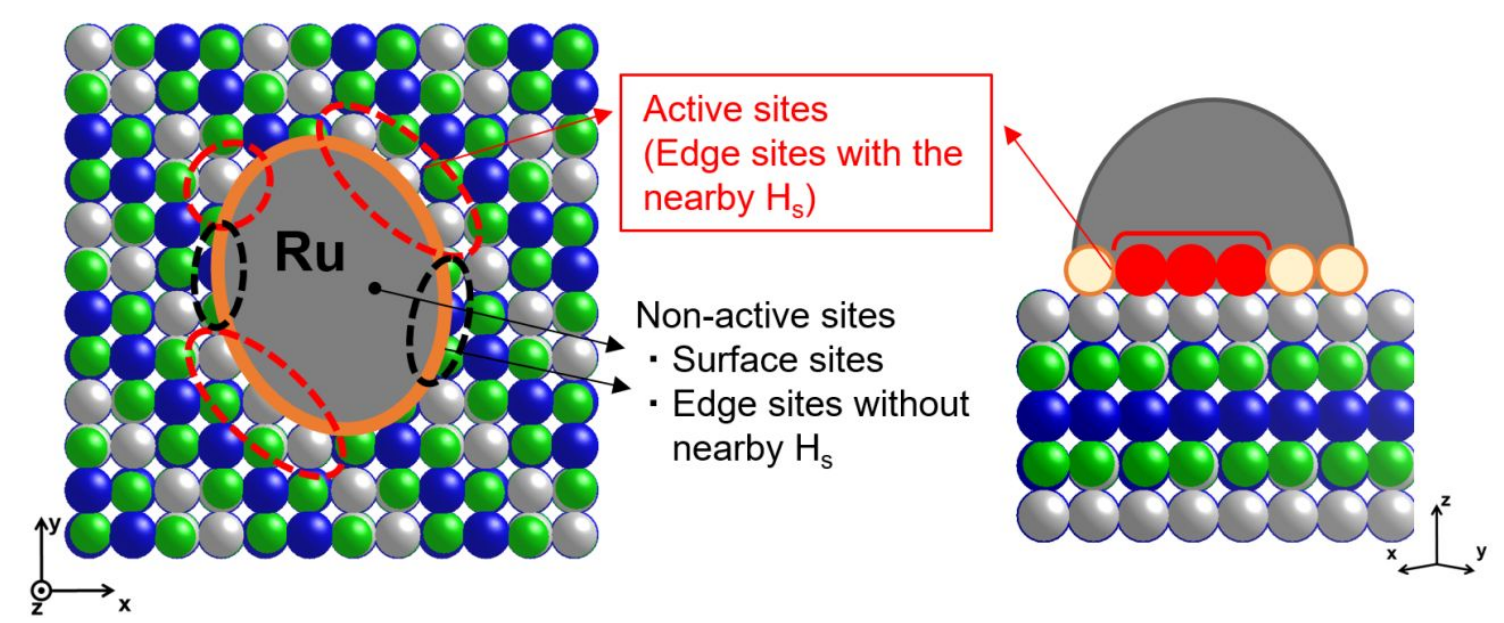

Figure S7. Top and side view of schematic images about reaction filed of $\mathrm{NH}_{3}$ synthesis using $\mathrm{Ru} / \mathrm{Ca}_{2} \mathrm{NH}_{1-x} \mathrm{e}_{x}^{-}$. 


\section{Turn over frequency}

The turn over frequency (TOF) obtained from the experiment can be expressed as:

$$
\mathrm{TOF}=r / n \#(\mathrm{~S} 10)
$$

where $r$ is the number of the produced ammonia molecules $\left(\mathrm{NH}_{3}\right)$ per unit time and unit mass of ruthenium $(\mathrm{Ru})$, and $n$ is the number of the active sites per unit mass of $\mathrm{Ru}$. The unit of TOF is site ${ }^{-1} \mathrm{~s}^{-1}$, and that of $r$ is $\mathrm{mol} \mathrm{g}^{-1} \mathrm{~s}^{-1}$, and that of $n$ is mol g-1, where site $\mathrm{e}^{-1}$ corresponds to the number of the produced $\mathrm{NH}_{3} \mathrm{~s}$ per the active site of $\mathrm{Ru}$.

We used the following Eq. S11 and S12 to count the number of the active sites of the $\mathrm{Ru}$ particles on $\mathrm{Ca}_{2} \mathrm{NH}$, where we assumed that the particle shape of $\mathrm{Ru}$ is semi-sphere. $n_{\text {edge }}$ and $n_{\text {surface }}$ are the numbers of the edge and surface sites of the Ru particle per unit mass of $\mathrm{Ru}$, and those are obtained as $\quad n_{\text {edge }}=\frac{12}{\rho d_{\mathrm{Ru}} d_{\mathrm{p}}^{2} N_{\mathrm{A}}} \#(\mathrm{~S} 11)$

$$
n_{\text {surface }}=\frac{6 N_{\text {surface }}}{\rho d_{\mathrm{p}} N_{\mathrm{A}}} \#(\mathrm{~S} 12)
$$

where $\rho$ is the density of $\mathrm{Ru}, d_{\mathrm{Ru}}$ is the distance of $\mathrm{Ru}-\mathrm{Ru}$ on $\mathrm{Ru}(0001)$ surface, $d_{\mathrm{p}}$ is the particle radius obtained by the experiment ${ }^{4}, N_{\mathrm{A}}$ is Avogadro constant, $N_{\text {surface }}$ is the number of the surface atoms on $\mathrm{Ru}(0001)$.

In this study, we calculated the TOF of the Ru edge sites by using the $r$ calculated from the values in Ref. 4 and the $n_{\text {edge }}$ obtained from Eq. 11 . We also compared the above TOF and the TOF obtained from microkinetic modeling using first principle calculations, and determined the rate-determining step of the ammonia synthesis over $\mathrm{Ru} / \mathrm{Ca}_{2} \mathrm{NH}$. 


\section{Coverage of adsorbed hydrogen and nitrogen atoms on Ru (0001)}

In order to investigate the coverage of adsorbed hydrogens and nitrogens on $\mathrm{Ru}$ surface, we calculated the grand potential per surface area for the hydrogen and nitrogen atoms on $\mathrm{Ru}(0001)$ as follows.

The chemical potential of gas-phase $\mathrm{X}_{2}\left(\mu_{\mathrm{X}_{2}}\right)(\mathrm{X}=\mathrm{H}, \mathrm{N})$ is expressed as:

$$
\mu_{\mathrm{X}_{2}}=E_{\mathrm{X}_{2}}+\left[H_{\mathrm{X}_{2}(\mathrm{~g})}^{0}(T)-H_{\mathrm{X}_{2}(\mathrm{~g})}^{0}(0 \mathrm{~K})\right]-T S_{\mathrm{X}_{2}(\mathrm{~g})}^{0}+k_{B} T \ln \left(p_{\mathrm{X}_{2}} / p_{\mathrm{X}_{2}}^{0}\right) \#(\mathrm{~S} 13
$$

where $E_{\mathrm{X}_{2}}$ is the calculated DFT energy of $\mathrm{X}_{2}$ in the gas-phase (at $0 \mathrm{~K}$, by definition), $H^{0}$ and $S^{0}$ are respectively the standard enthalpy and entropy values of gas-phase $\mathrm{X}_{2}$ obtained from the NIST-JANAF Thermochemical Tables ${ }^{5}, p_{\mathrm{X}_{2}}$ is the partial pressure of $\mathrm{X}_{2}$, and the standard state pressure $p_{\mathrm{X}_{2}}^{0}$ is $=1$ bar.

The chemical potential of surface $X^{*}\left(\mu_{X^{*}}\right)$ is related to that of gas-phase $X_{2}$ through the equilibrium $\mathrm{X}_{2}(\mathrm{~g})+2 * \leftrightarrow 2 \mathrm{X}^{*}$ (* denotes an adsorption site) according to Eq. S14:

$$
\mu_{\mathrm{X}_{2}}=2 \mu_{\mathrm{X} *} \#(\mathrm{~S} 14)
$$

Comparing of Eq. S13 and Eq. S14, the chemical potential of surface $\mathrm{X}^{*}$ is expressed as:

$$
\mu_{\mathrm{X} *}=\frac{1}{2}\left\{E_{\mathrm{X}_{2}}+\left[H_{\mathrm{X}_{2}(\mathrm{~g})}^{0}(T)-H_{\mathrm{X}_{2}(\mathrm{~g})}^{0}(0 \mathrm{~K})\right]-T S_{\mathrm{X}_{2}(\mathrm{~g})}^{0}+k_{B} T \ln \left(p_{\mathrm{X}_{2}} / p_{\mathrm{X}_{2}}^{0}\right)\right\} \#(\mathrm{~S} 15)
$$

The grand potential per surface area $(\Omega)$ for the hydrogen and nitrogen atoms on $\mathrm{Ru}$ (0001) at each surface coverage is expressed as:

$$
\begin{aligned}
& \Omega\left(p_{\mathrm{X}_{2}}, T\right)=\frac{1}{A}\left(E_{\mathrm{nX}+\text { slab }}-E_{\text {slab }}-n \mu_{\mathrm{X} *}\right) \\
& =\frac{1}{A}\left(\Delta E_{\mathrm{nX}}-\frac{n}{2}\left\{k_{B} T \ln \left(p_{\mathrm{X}_{2}} / p_{\mathrm{X}_{2}}^{0}\right)+\left[H_{\mathrm{X}_{2}(\mathrm{~g})}^{0}(T)-H_{\mathrm{X}_{2}(\mathrm{~g})}^{0}(0 \mathrm{~K})\right]-T S_{\mathrm{X}_{2}(\mathrm{~g})}^{0}\right\}\right) \\
& \Delta E_{\mathrm{nX}}=E_{\mathrm{nX}+\mathrm{slab}}-E_{\mathrm{slab}}-\frac{n}{2} E_{\mathrm{X}_{2}} \#(\mathrm{~S} 17)
\end{aligned}
$$

where $n$ is the number of $\mathrm{X}$ atoms $(n(\mathrm{X}))$ in the unit cell, $E_{\mathrm{nX}+\text { slab }}$ is the total energy of $n \mathrm{X}$ atoms adsorbed on the slab model of Ru (0001), $E_{\text {slab }}$ is the total energy of the slab model of $\mathrm{Ru}(0001), \Delta E_{\mathrm{nX}}$ is the adsorption energy of $n \mathrm{X}$ atoms on $\mathrm{Ru}(0001)$, and $A$ is the surface area. Note that the surface entropy of hydrogen or nitrogen is almost constant with regardless of the adsorption structures in the temperature range of interest $\left(300-400^{\circ} \mathrm{C}\right)$, 
and therefore we omitted the entropy differences related to the surface entropies in Eq. $\mathrm{S} 16$. At a given temperature and pressure, the coverage of $\mathrm{H}$ or $\mathrm{N}$ is determined as the lowest grand potential per surface area in all the calculated $\mathrm{H}$ or $\mathrm{N}$ coverages.

Figure S8 shows the configurations and grand potentials per surface area $(\Omega)$ for $\mathrm{H}$ and $\mathrm{N}$ atoms on $\mathrm{Ru}(0001)$ at different $\mathrm{H}$ and $\mathrm{N}$ coverages as a function of temperature. Under the experimental hydrogen and nitrogen pressure $(0.75$ and 0.25 bar, respectively) and at the temperature $\left(300-400^{\circ} \mathrm{C}\right)$, the $n(\mathrm{H})$ and $n(\mathrm{~N})$ on $\mathrm{Ru}(0001)$ is 16 and 4 per $10.884 \AA \times 9.436 \AA$, respectively.

a

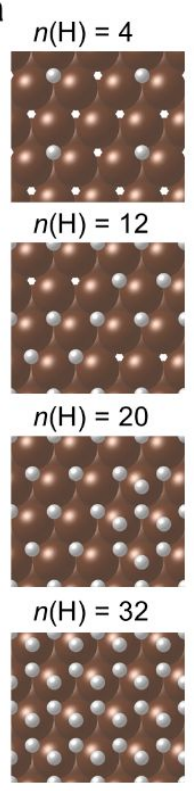

b

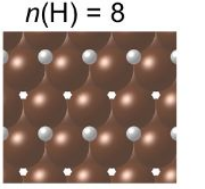

$n(\mathrm{H})=16$

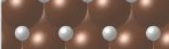

0. 0.0.

0.0 .0 .0$.

$n(\mathrm{H})=24$

0.0 .0$.

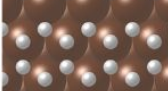

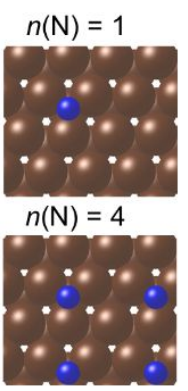

$n(\mathrm{~N})=12$

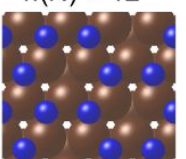

$n(\mathrm{~N})=2$

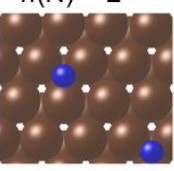

$n(\mathrm{~N})=8$

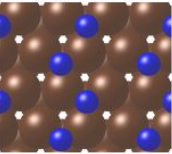

$n(\mathrm{~N})=16$

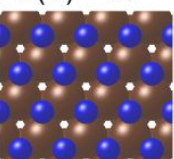

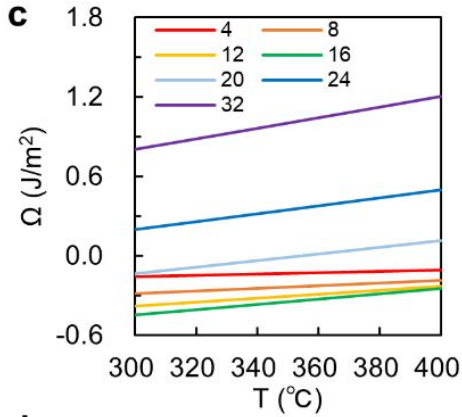

d

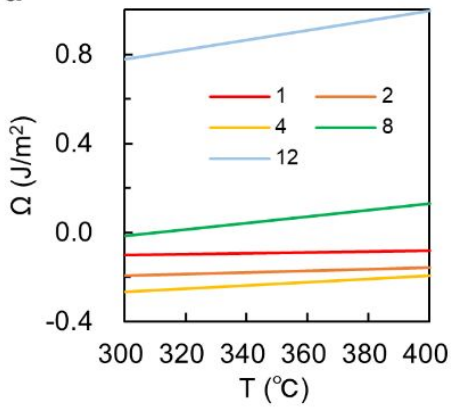

Figure S8. $(\mathrm{a}, \mathrm{b})$ Configurations, and $(\mathrm{c}, \mathrm{d})$ grand potentials per surface area $(\Omega)$ for $\mathrm{H}$ and $\mathrm{N}$ atoms on $\mathrm{Ru}(0001)$ when the $n(\mathrm{H})$ is $4,8,12,16,20,24,32$, and the $n(\mathrm{~N})$ is 1,2 , $4,8,12,16$ per $10.884 \AA \times 9.436 \AA$ as a function of temperature when $p_{\mathrm{H}_{2}}$ is 0.75 bar and $p_{\mathrm{N}_{2}}$ is 0.25 bar, respectively. White, blue and blown balls represent $\mathrm{H}, \mathrm{N}$ and $\mathrm{Ru}$, respectively. 


\section{Rate determining step of $\mathrm{NH}_{3}$ synthesis with an additional $\mathrm{H}$ on the surface of $\mathrm{Ru}_{5}$ cluster}

Table S4 shows the activation energies for the forward and backward reactions $\left(E_{\mathrm{a}, \text { for }} / E_{\mathrm{a}, \mathrm{rev}}\right)$ of the $\mathrm{NH}_{x}$ formation and $\mathrm{H}$ migration into $\mathrm{V}_{\mathrm{H}}$ with an additional $\mathrm{H}$ on the surface of $\mathrm{Ru}_{5}$ cluster. Figure $\mathrm{S} 9$ also shows the calculated TOF for each elementary step by using the activation energies with the additional $\mathrm{H}$ on the surface of $\mathrm{Ru}_{5}$ cluster.

Table S4. Activation energy for the forward and backward reactions ( $\left.E_{\mathrm{a}, \text { for }} / E_{\mathrm{a}, \mathrm{rev}}\right)$ of the $\mathrm{NH}_{x}$ formation and $\mathrm{H}$ migration into $\mathrm{V}_{\mathrm{H}}$ with an additional $\mathrm{H}$ on the surface of $\mathrm{Ru}_{5}$ cluster.

\begin{tabular}{ccc}
\hline Elementary step & $E_{\mathrm{a}, \text { for }}[\mathrm{eV}]$ & $E_{\mathrm{a}, \text { rev }}[\mathrm{eV}]$ \\
\hline $\mathrm{N}^{*}+\mathrm{H}_{\mathrm{s}} \rightarrow \mathrm{NH}^{*}+\mathrm{V}_{\mathrm{H}}$ & 0.88 & 1.60 \\
$\mathrm{NH}^{*}+\mathrm{H}_{\mathrm{s}} \rightarrow \mathrm{NH}_{2}^{*}+\mathrm{V}_{\mathrm{H}}$ & 1.16 & 1.16 \\
$\mathrm{NH}_{2}^{*}+\mathrm{H}_{\mathrm{s}} \rightarrow \mathrm{NH}_{3}^{*}+\mathrm{V}_{\mathrm{H}}$ & 1.13 & 2.22 \\
$\mathrm{H}^{*}+\mathrm{V}_{\mathrm{H}} \rightarrow \mathrm{H}_{\mathrm{s}}+*$ & 0.48 & 0.57 \\
\hline
\end{tabular}




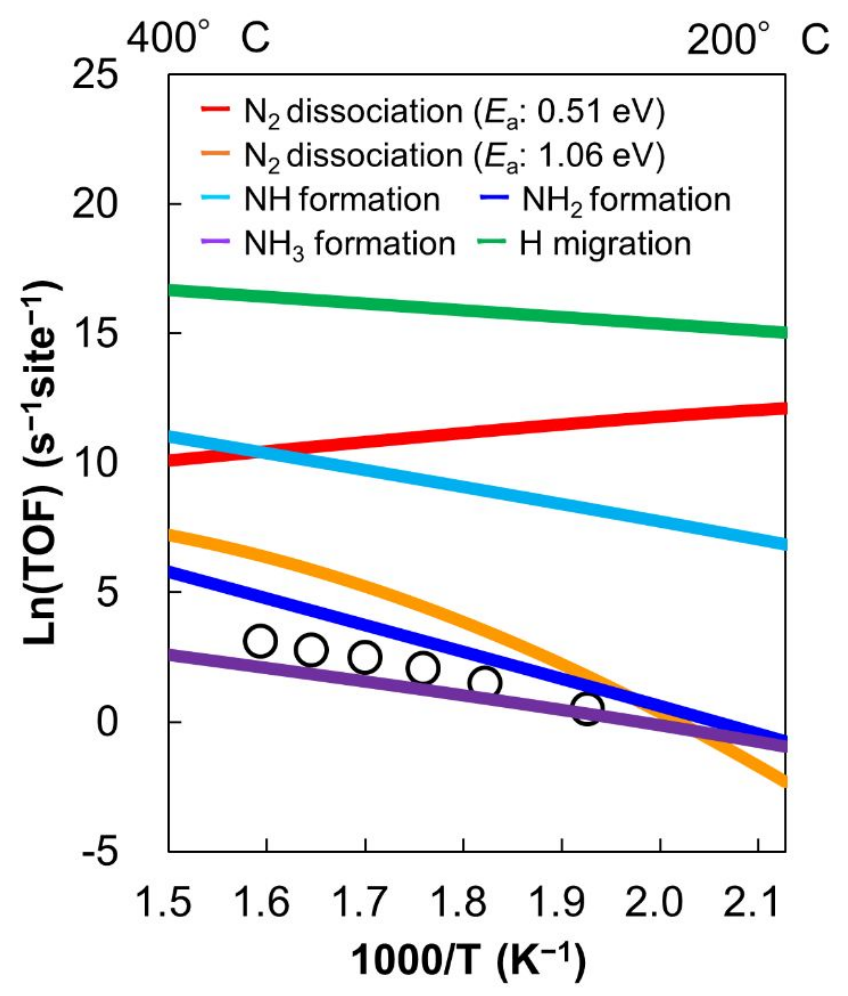

Figure S9. Calculated TOF for $\mathrm{NH}_{3}$ synthesis on $\mathrm{Ru} / \mathrm{Ca}_{2} \mathrm{NH}$ by microkinetic modeling. The activation energies of the $\mathrm{NH}_{x}$ formation and $\mathrm{H}$ migration into $\mathrm{V}_{\mathrm{H}}$ with an additional $\mathrm{H}$ on the surface of $\mathrm{Ru}_{5}$ cluster are used. Each line shows the calculated TOF when an elementary step such as $\mathrm{N}_{2}$ dissociation, $\mathrm{NH}_{x}$ formation, $\mathrm{H}$ migration, etc. is assumed to be the RDS. The circles (white) indicate the TOF calculated from the activity rate obtained experimentally ${ }^{4}$, where the active site was assumed to be the edge of hemispherical particles. 


\section{Sensitivity of the activation energies with DFT calculations on RDS determined with the microkinetic modeling}

Figure $\mathrm{S} 10$ shows the calculated TOFs for $\mathrm{NH}_{3}$ synthesis on $\mathrm{Ru} / \mathrm{Ca}_{2} \mathrm{NH}$ with

microkinetic modeling by changing the activation energies from DFT results by $+/-20 \%$ at most.

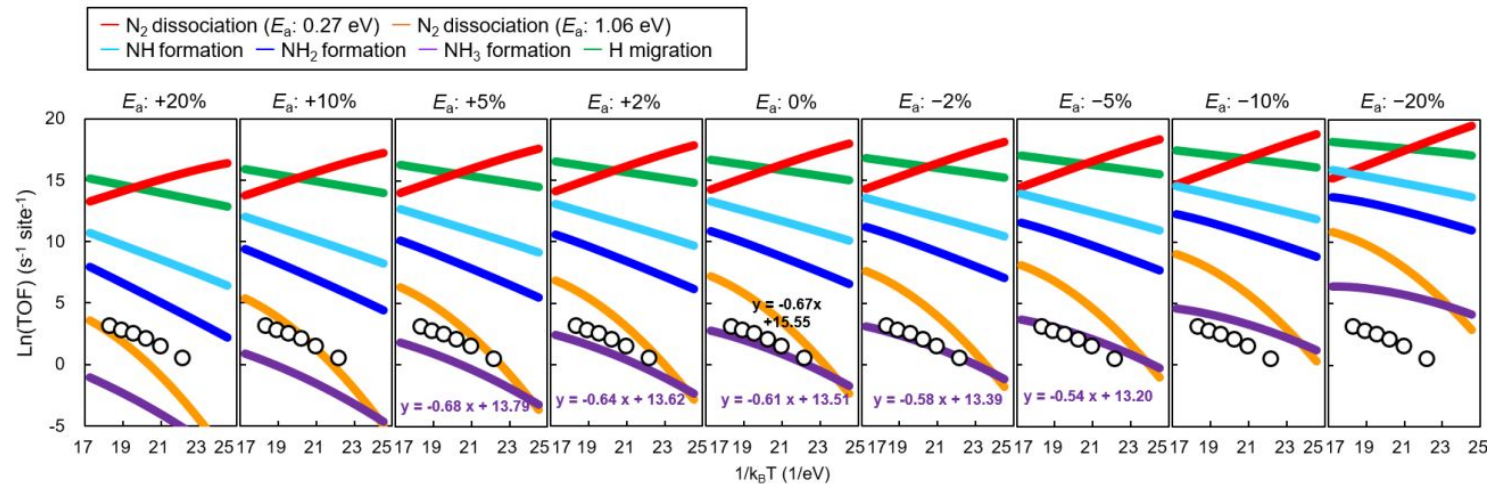

Figure S10. Calculated TOFs for $\mathrm{NH}_{3}$ synthesis on $\mathrm{Ru} / \mathrm{Ca}_{2} \mathrm{NH}$ with microkinetic modeling by changing the activation energies from DFT results by $+/-20 \%$ at most. 


\section{Atomic coordinates of the elementary steps of $\mathrm{NH}_{3}$ synthesis on $\mathbf{R u} / \mathrm{Ca}_{2} \mathbf{N H}$}

Table S5: Fractional atomic coordinates of the initial state of the $\mathrm{N}_{2}$ dissociation around $2 \mathrm{~V}_{\mathrm{H}}$ on Ru$/ \mathrm{Ca}_{2} \mathrm{NH}$. Lattice parameters are: $\mathrm{a}=\mathrm{b}=10.1067 \AA, \mathrm{c}=39.0701 \AA, \alpha=\beta=\gamma$ $=90^{\circ}$.

$\begin{array}{ccccc}\text { No. } & \text { Atom } & X & Y & Z \\ 1 & \mathrm{Ca} & 0.108186341016477 & 0.391813636918875 & 0.030294838468024 \\ 2 & \mathrm{Ca} & 0.391813636918875 & 0.108186341016477 & 0.030294838468024 \\ 3 & \mathrm{Ca} & 0.608186393358167 & 0.891813666998032 & 0.030294838468024 \\ 4 & \mathrm{Ca} & 0.891813666998032 & 0.608186393358167 & 0.030294838468024 \\ 5 & \mathrm{Ca} & 0.139695440095728 & 0.860304515676034 & 0.035014432581349 \\ 6 & \mathrm{Ca} & 0.360304545260469 & 0.639695485016574 & 0.035014432581349 \\ 7 & \mathrm{Ca} & 0.639695485016574 & 0.360304545260469 & 0.035014432581349 \\ 8 & \mathrm{Ca} & 0.860304515676034 & 0.139695440095728 & 0.035014432581349 \\ 9 & \mathrm{Ca} & 0.136710031423064 & 0.636710076343902 & 0.094508290935160 \\ 10 & \mathrm{Ca} & 0.363289953933140 & 0.863289924348706 & 0.094508290935160 \\ 11 & \mathrm{Ca} & 0.636710076343902 & 0.136710031423064 & 0.094508290935160 \\ 12 & \mathrm{Ca} & 0.863289924348706 & 0.363289953933140 & 0.094508290935160 \\ 13 & \mathrm{Ca} & 0.113472400636908 & 0.113472400636908 & 0.099069771470752 \\ 14 & \mathrm{Ca} & 0.386527577298444 & 0.386527577298444 & 0.099069771470752 \\ 15 & \mathrm{Ca} & 0.613472452978598 & 0.613472452978598 & 0.099069771470752 \\ 16 & \mathrm{Ca} & 0.886527607377601 & 0.886527607377601 & 0.099069771470752 \\ 17 & \mathrm{Ca} & 0.112507753199288 & 0.887492254914164 & 0.158071913907946 \\ 18 & \mathrm{Ca} & 0.387492224835007 & 0.612507805540978 & 0.158071913907946 \\ 19 & \mathrm{Ca} & 0.612507805540978 & 0.387492224835007 & 0.158071913907946 \\ 20 & \mathrm{Ca} & 0.887492254914164 & 0.112507753199288 & 0.158071913907946 \\ 21 & \mathrm{Ca} & 0.136566124331452 & 0.364201415726107 & 0.164560744241143 \\ 22 & \mathrm{Ca} & 0.364201415726107 & 0.136566124331452 & 0.164560744241143 \\ 23 & \mathrm{Ca} & 0.635798614550935 & 0.863433831440318 & 0.164560744241143 \\ 24 & \mathrm{Ca} & 0.863433831440318 & 0.635798614550935 & 0.164560744241143 \\ & \mathrm{Ca} & 0.137168778578413 & 0.137168778578413 & 0.222797289988278 \\ & \mathrm{Ca} & 0.363915689045413 & 0.363915689045413 & 0.222744141748108 \\ 29 & 0.636084341231629 & 0.636084341231629 & 0.222744141748108 \\ & 0.862831177193356 & 0.862831177193356 & 0.222797289988278 \\ 25 & 0.111699362916830 & 0.614895043217814 & 0.229141570738541\end{array}$




\begin{tabular}{|c|c|c|c|c|}
\hline & $\mathrm{Ca}$ & 0.385104987059222 & 0.888300645097679 & \\
\hline & $\mathrm{Ca}$ & 0.614895043217814 & 0.111699362916830 & 0.229141570738541 \\
\hline & $\mathrm{Ca}$ & .888300645097679 & 0.385104987059222 & 0.229141570738541 \\
\hline & $\mathrm{Ca}$ & .115278093063452 & .387513743516890 & 0.287674809439067 \\
\hline & $\mathrm{Ca}$ & 0.387513743516890 & 0.115278093063452 & 0.287674809439067 \\
\hline & $\mathrm{Ca}$ & & & \\
\hline & $\mathrm{Ca}$ & 0.884721914951057 & 0.612486286760152 & 0.287674809439067 \\
\hline & $\mathrm{Ca}$ & & & \\
\hline & $\mathrm{Ca}$ & .358954529519735 & 0.641045500757314 & 0.293137733454500 \\
\hline & $\mathrm{Ca}$ & & & \\
\hline & $\mathrm{Ca}$ & .867540559546640 & 0.132459396225129 & 0.295239692459809 \\
\hline & $\mathrm{Ca}$ & & & \\
\hline & $\mathrm{Ca}$ & .360522558648710 & 0.870576337138096 & 0.352154216036922 \\
\hline & $\mathrm{Ca}$ & 0.639477471628332 & 0.129423618633673 & 4216036922 \\
\hline & $\mathrm{Ca}$ & .870576337138096 & .360522558648710 & 6922 \\
\hline & $\mathrm{Ca}$ & 0.115420889015332 & 0.115420889015332 & 0.364497519491820 \\
\hline 46 & $\mathrm{Ca}$ & 0.391885891540468 & 0.391885891540468 & 2469880930 \\
\hline 47 & $\mathrm{Ca}$ & 0.608114138835517 & 35517 & 2469880930 \\
\hline & $\mathrm{Ca}$ & 0.884579119098127 & 0.884579119098127 & 0.364497519491820 \\
\hline & & & 5413 & 1408 \\
\hline 50 & $\mathrm{Ca}$ & 0.350384680917066 & 0.649615349359983 & 0.412504117199169 \\
\hline & & & 066 & 9169 \\
\hline 52 & $\mathrm{Ca}$ & 0.892311223355413 & 0.107688784659096 & 0.424895404571408 \\
\hline & $\mathrm{Ca}$ & 0.158243500287866 & 310549447 & 69064626 \\
\hline & $\mathrm{Ca}$ & 383262310549447 & 0.158243500287866 & \\
\hline 55 & $\mathrm{Ca}$ & 727595 & אנסדנתדשה & 69064626 \\
\hline 56 & $\mathrm{Ca}$ & 0.841756455483903 & 0.616737719727595 & 0.427282869064626 \\
\hline 57 & $\mathrm{Ca}$ & 0.193911841292980 & 0.193911841292980 & 0.490387700266879 \\
\hline 58 & $\mathrm{Ca}$ & .806088114478789 & .806088114478789 & 0.490387700266879 \\
\hline 59 & $\mathrm{Ca}$ & 0.084863293365395 & 0.637709675187842 & \\
\hline 60 & $\mathrm{Ca}$ & 0.362290355089207 & 0.915136714649122 & 0.492235666447626 \\
\hline 61 & $\mathrm{Ca}$ & 0.637709675187842 & 0.084863293365395 & \\
\hline 62 & $\mathrm{Ca}$ & 0.915136714649122 & 0.362290355089207 & 0.492235666447626 \\
\hline 63 & $\mathrm{~N}$ & 0.121532366256275 & 0.621532366454168 & 0.0347428815803 \\
\hline 64 & $\mathrm{~N}$ & 0.378467634040553 & 0.878467634238447 & 0.03474288158033 \\
\hline
\end{tabular}




\begin{tabular}{|c|c|c|c|c|}
\hline & $\mathrm{N}$ & 0.621532366454168 & 0.121532366256275 & 0.034742881580335 \\
\hline & $\mathrm{N}$ & .878467634238447 & 0.378467634040553 & 0.034742881580335 \\
\hline & $\mathrm{N}$ & 0.124450822091830 & 0.875549178402891 & 0.096328359639614 \\
\hline & $\mathrm{N}$ & .375549178205005 & 0.624450822289716 & .096328359639614 \\
\hline 69 & $\mathrm{~N}$ & 0.624450822289716 & 0.375549178205005 & 0.096328359639614 \\
\hline & $\mathrm{N}$ & & & 0.096328359639614 \\
\hline & $\mathrm{N}$ & 0.125158390898768 & 0.125158390898768 & 0.161315821650490 \\
\hline & $\mathrm{N}$ & 0.374841609398060 & 0.374841609398060 & .161315821650490 \\
\hline & $\mathrm{N}$ & 0.625158391096661 & 0.625158391096661 & 0.161315821650490 \\
\hline 74 & $\mathrm{~N}$ & 0.874841609595954 & 0.874841609595954 & 0.161315821650490 \\
\hline & $\mathrm{N}$ & 0.125716588735272 & 0.376218416115051 & 0.226719564153825 \\
\hline 76 & $\mathrm{~N}$ & 0.376218416115051 & 0.125716588735272 & 0.226719564153825 \\
\hline & $\mathrm{N}$ & 0.623781584379671 & 0.874283411759449 & 0.226719564153825 \\
\hline 78 & $\mathrm{~N}$ & 0.874283411759449 & 0.623781584379671 & 0.226719564153825 \\
\hline 79 & $\mathrm{~N}$ & 0.120501324742278 & 0.629878019448540 & 0.291490037634950 \\
\hline 80 & $\mathrm{~N}$ & 0.370121981046182 & 0.879498675752451 & 0.291490037634950 \\
\hline 81 & $\mathrm{~N}$ & 0.629878019448540 & 0.120501324742278 & 0.291490037634950 \\
\hline 82 & $\mathrm{~N}$ & 0.879498675752451 & 0.370121981046182 & 0.291490037634950 \\
\hline 83 & $\mathrm{~N}$ & 0.122465758777195 & 0.877534241717526 & 0.361302782849136 \\
\hline 84 & $\mathrm{~N}$ & 3949966 & 630199766544755 & 10279172 \\
\hline 85 & $\mathrm{~N}$ & 0.630199766544755 & 0.369800233949966 & 0.353414510279172 \\
\hline 86 & $\mathrm{~N}$ & 0.877534241 & 5758777195 & 82849136 \\
\hline 87 & $\mathrm{~N}$ & 0.139305632116531 & 0.139305632116531 & 0.429594910288891 \\
\hline 88 & $\mathrm{~N}$ & 0.391507810655795 & 0.391507810655795 & 583490950515 \\
\hline 89 & $\mathrm{~N}$ & 0.608492189838926 & 0.608492189838926 & 0.416583490950515 \\
\hline 90 & $\mathrm{~N}$ & 0.860694368378190 & 0.860694368378190 & 0.429594910288891 \\
\hline 91 & $\mathrm{~N}$ & 0.140829131099195 & 0.417028749846856 & 0.489520146363325 \\
\hline 92 & $\mathrm{~N}$ & 0.417028749846856 & 0.140829131099195 & 0.489520146363325 \\
\hline 93 & $\mathrm{~N}$ & 0.582971250647865 & 0.859170869395526 & 0.489520146363325 \\
\hline 94 & $\mathrm{~N}$ & 0.859170869395526 & 0.582971250647865 & 0.489520146363325 \\
\hline 95 & $\mathrm{~N}$ & 0.548251330688010 & 0.451748699589039 & 0.533929437796274 \\
\hline 96 & $\mathrm{~N}$ & 0.451748699589039 & 0.548251330688010 & 0.533929437796274 \\
\hline 97 & $\mathrm{H}$ & 0.133716503752943 & 0.133716503752943 & 0.032444094734203 \\
\hline 98 & $\mathrm{H}$ & 0.366283496543886 & 0.366283496543886 & 0.03244409473420 \\
\hline 99 & $\mathrm{H}$ & 0.633716503950836 & 0.633716503950836 & 0.032444094734203 \\
\hline
\end{tabular}




$\begin{array}{lllll}100 & \mathrm{H} & 0.866283496741779 & 0.866283496741779 & 0.032444094734203 \\ 101 & \mathrm{H} & 0.125126018899749 & 0.625126019097635 & 0.161484969662801 \\ 102 & \mathrm{H} & 0.374873981397087 & 0.874873981594973 & 0.161484969662801 \\ 103 & \mathrm{H} & 0.625126019097635 & 0.125126018899749 & 0.161484969662801 \\ 104 & \mathrm{H} & 0.874873981594973 & 0.374873981397087 & 0.161484969662801 \\ 105 & \mathrm{H} & 0.121891089370279 & 0.878108911124442 & 0.227849964313158 \\ 106 & \mathrm{H} & 0.374429118933107 & 0.625570881561614 & 0.227011642514447 \\ 107 & \mathrm{H} & 0.625570881561614 & 0.374429118933107 & 0.227011642514447 \\ 108 & \mathrm{H} & 0.878108911124442 & 0.121891089370279 & 0.227849964313158 \\ 109 & \mathrm{H} & 0.124886858446188 & 0.124886858446188 & 0.293488357986440 \\ 110 & \mathrm{H} & 0.375092893631517 & 0.375092893631517 & 0.291069402517784 \\ 111 & \mathrm{H} & 0.624907106863204 & 0.624907106863204 & 0.291069402517784 \\ 112 & \mathrm{H} & 0.875113142048533 & 0.875113142048533 & 0.293488357986440 \\ 113 & \mathrm{H} & 0.128938665580591 & 0.375882354645476 & 0.358044217403758 \\ 114 & \mathrm{H} & 0.375882354645476 & 0.128938665580591 & 0.358044217403758 \\ 115 & \mathrm{H} & 0.624117645849246 & 0.871061334914131 & 0.358044217403758 \\ 116 & \mathrm{H} & 0.871061334914131 & 0.624117645849246 & 0.358044217403758 \\ 117 & \mathrm{H} & 0.109524510692452 & 0.627649697474190 & 0.422097590412661 \\ 118 & \mathrm{H} & 0.372350303020539 & 0.890475489802270 & 0.422097590412661 \\ 119 & \mathrm{H} & 0.627649697474190 & 0.109524510692452 & 0.422097590412661 \\ 120 & \mathrm{H} & 0.890475489802270 & 0.372350303020539 & 0.422097590412661 \\ 121 & \mathrm{H} & 0.133673832250309 & 0.866326168244413 & 0.487428827511813 \\ 122 & \mathrm{H} & 0.866326168244413 & 0.133673832250309 & 0.487428827511813 \\ 123 & \mathrm{Ru} & 0.500000030079156 & 0.500000030079156 & 0.450205640743575 \\ 124 & \mathrm{Ru} & 0.321323479538534 & 0.487374911382019 & 0.497410400539315 \\ 125 & \mathrm{Ru} & 0.487374911382019 & 0.321323479538534 & 0.497410400539315 \\ 126 & \mathrm{Ru} & 0.512625148677351 & 0.678676520956187 & 0.497410400539315 \\ 127 & \mathrm{Ru} & 0.678676520956187 & 0.512625148677351 & 0.497410400539315\end{array}$

Table S6: Fractional atomic coordinates of the final state of the $\mathrm{N}_{2}$ dissociation around $2 \mathrm{~V}_{\mathrm{H}}$ on $\mathrm{Ru} / \mathrm{Ca}_{2} \mathrm{NH}$. Lattice parameters are: $\mathrm{a}=\mathrm{b}=10.1067 \AA, \mathrm{c}=39.0701 \AA, \alpha=\beta=\gamma$ $=90^{\circ}$.
No. Atom
$X$
$Y$
$Z$
$1 \quad \mathrm{Ca} \quad 0.108186341016477$
0.391813636918875
0.030294838468024
$2 \mathrm{Ca} \quad 0.391813636918875$
0.108186341016477
0.030294838468024 


\begin{tabular}{|c|c|c|c|}
\hline $\mathrm{Ca}$ & & & \\
\hline $\mathrm{Ca}$ & 0.891813666998032 & 0.608186393358167 & 002070402016802 \\
\hline $\mathrm{Ca}$ & .139695440095728 & 0.860304515676034 & 00250144325812 \\
\hline $\mathrm{Ca}$ & 260469 & 5574 & \\
\hline $\mathrm{Ca}$ & 0.639695485016574 & 0.360304545260469 & 0.035014432581349 \\
\hline $\mathrm{Ca}$ & 5034 & 5728 & \\
\hline $\mathrm{Ca}$ & 0.136710031423064 & 0.636710076343902 & 0.094508290935160 \\
\hline $\mathrm{Ca}$ & & & \\
\hline $\mathrm{Ca}$ & & & 5160 \\
\hline $\mathrm{Ca}$ & 0.863289924348706 & 0.363289953933140 & 35160 \\
\hline $\mathrm{Ca}$ & 0.113472400636908 & & 0752 \\
\hline $\mathrm{Ca}$ & & & \\
\hline $\mathrm{Ca}$ & 0.613472452978598 & 0.6134 & \\
\hline $\mathrm{Ca}$ & 0.886527607377601 & 77601 & \\
\hline $\mathrm{Ca}$ & 0.112507753199288 & $0.887492254 \mathrm{C}$ & 907946 \\
\hline $\mathrm{Ca}$ & & & \\
\hline $\mathrm{Ca}$ & 0.612507805540978 & 835007 & \\
\hline $\mathrm{Ca}$ & & & \\
\hline $\mathrm{Ca}$ & 0.137072151 & 507 & \\
\hline $\mathrm{Ca}$ & 0.362 & & \\
\hline $\mathrm{Ca}$ & & & \\
\hline $\mathrm{Ca}$ & 0.862927804358079 & 0.637072196334536 & 3173 \\
\hline $\mathrm{Ca}$ & 0.137041882792495 & 0.137041882792495 & 3636 \\
\hline $\mathrm{Ca}$ & 0.363729408190614 & 0.363729408190614 & \\
\hline $\mathrm{Ca}$ & 86435 & & \\
\hline $\mathrm{Ca}$ & & & \\
\hline $\mathrm{Ca}$ & & & \\
\hline$C a$ & & & \\
\hline $\mathrm{Ca}$ & & & 0.22 \\
\hline $\mathrm{Ca}$ & 0.888329931958374 & 0.385278204751408 & 0.229249671369466 \\
\hline $\mathrm{Ca}$ & 0.114970736049905 & 0.387538953818733 & 0.2878 \\
\hline $\mathrm{Ca}$ & 0.387538953818733 & 0.114970736049905 & 0.287843243451668 \\
\hline $\mathrm{Ca}$ & 0.612461076458309 & 0.885029271964604 & 0.2878432434516 \\
\hline $\mathrm{Ca}$ & 0.885029271964604 & 0.612461076458309 & 0.287843243451 \\
\hline $\mathrm{Ca}$ & 0.132768189296669 & 0.867231766475100 & 0.29553329104524 \\
\hline
\end{tabular}




\begin{tabular}{|c|c|c|c|c|}
\hline & $\mathrm{Ca}$ & & & \\
\hline & $\mathrm{Ca}$ & .640537702362642 & 0.359462327914407 & 0.29301289302079 \\
\hline & & 867231766475100 & .132768189296669 & 0.29553329104524 \\
\hline & $\mathrm{Ca}$ & & & ב \\
\hline & $\mathrm{Ca}$ & .360999310226958 & 0.868660577282881 & 0.35239855056340 \\
\hline & $\mathrm{Ca}$ & & & 340 \\
\hline & $\mathrm{Ca}$ & 868660577282881 & 999310226958 & 0.35239855056340 \\
\hline & $\mathrm{Ca}$ & & & \\
\hline & $\mathrm{Ca}$ & & & \\
\hline & & 79527 & & 0.3575 \\
\hline & $\mathrm{Ca}$ & & 0.884074200705150 & \\
\hline & & & & \\
\hline & $\mathrm{Ca}$ & 317 & & \\
\hline & $\mathrm{Ca}$ & 1726 & 53475317 & 0.41 \\
\hline & $\mathrm{Ca}$ & 890260050266881 & 0.109739957747635 & 0.42335 \\
\hline & & & & \\
\hline & $\mathrm{Ca}$ & 0.381862648911301 & & \\
\hline & $\mathrm{Ca}$ & & & \\
\hline & & & & \\
\hline & $\mathrm{Ca}$ & & & \\
\hline & $\mathrm{Ca}$ & & & \\
\hline & $\mathrm{Ca}$ & 0.090408791322616 & & \\
\hline & & & 92 & 0.4 \\
\hline & $\mathrm{Ca}$ & & & 0.491400039 \\
\hline & $\mathrm{Ca}$ & & & 0.4 \\
\hline & $\mathrm{N}$ & & & \\
\hline & $\mathrm{N}$ & & & 0.0 \\
\hline & & & & \\
\hline 66 & $\mathrm{~N}$ & 8467634238447 & 0.378467634040553 & 0.0347428 \\
\hline & $\mathrm{N}$ & 0.124450822091830 & 0.875549178402891 & 0.09632835963961 \\
\hline 68 & $\mathrm{~N}$ & 75549178205005 & 0.624450822289716 & \\
\hline 69 & $\mathrm{~N}$ & 0.624450822289716 & 0.375549178205005 & 0.09632835963961 \\
\hline 70 & $\mathrm{~N}$ & 0.875549178402891 & 0.124450822091830 & \\
\hline 71 & $\mathrm{~N}$ & 0.125158390898768 & 0.125158390898768 & 0.16131582165049 \\
\hline & $\mathrm{N}$ & 0.374841609398060 & 0.374841609398060 & 0.1613158216504 \\
\hline
\end{tabular}




\begin{tabular}{|c|c|c|c|c|}
\hline & & 25158391096661 & 525158391096661 & \\
\hline & $\mathrm{N}$ & 0.874841609595954 & 0.874841609595954 & 0.16131582165049 \\
\hline & & .125765350352281 & 0.375856538610186 & 022658838743032 \\
\hline & & 0.375856538610186 & 0.125765350 & 0.2265 \\
\hline & & .624143461884536 & 0.874234650142440 & 0.22658838743032 \\
\hline & $\mathrm{N}$ & 440 & 84536 & 0.2265883874 \\
\hline & $\mathrm{N}$ & 0.120598873453026 & 0.629293150916176 & 0.29145861043036 \\
\hline & $\mathrm{N}$ & 370706849578546 & 0.879401 & 0.291 \\
\hline & $\mathrm{N}$ & & & \\
\hline & $\mathrm{N}$ & 0.879401127041695 & 0.370706849578546 & 0.29145861043036 \\
\hline & $\mathrm{N}$ & 0.122528867779273 & 0.877471132715449 & 0.36060 \\
\hline & $\mathrm{N}$ & 0.370463983135159 & 0.629536017359562 & 0.35282472026001 \\
\hline & $\mathrm{N}$ & 0.629536017359562 & 0.370463983135159 & 0.3528 \\
\hline & $\mathrm{N}$ & 0.877471132715449 & 0.122528867779273 & 0.36060918433668 \\
\hline & $\mathrm{N}$ & 0.136954071425847 & 0.136954071425847 & 0.42881954684846 \\
\hline & & & & 0.41 \\
\hline & $\mathrm{N}$ & 0.605706857354277 & 0.605706857354277 & 0.41737999280027 \\
\hline & $\mathrm{N}$ & 0.863045929068874 & 0.863045929068874 & 0.4288 \\
\hline & & & & 0.49 \\
\hline & $\mathrm{N}$ & 0.402172473539153 & & 0.490 \\
\hline & $\mathrm{N}$ & 0.597827526955569 & 0.864546104536280 & 0.49031332765872 \\
\hline & $\mathrm{N}$ & 0.864546104536280 & 0.597827526955569 & 0.49031332765872 \\
\hline & $\mathrm{N}$ & 0.344705131787 & 0.6552948 & 0.50773245426177 \\
\hline & $\mathrm{N}$ & 0.655294898588600 & 0.344705131787385 & \\
\hline & $\mathrm{H}$ & 0.13 & & 420 \\
\hline & $\mathrm{H}$ & 0.366283496543886 & 0.366283496543886 & 0.03244409473420 \\
\hline & $\mathrm{H}$ & 36 & 0.6 & \\
\hline & $\mathrm{H}$ & & & 0.03244409473420 \\
\hline & $\mathrm{H}$ & 0.125126018899749 & 0.625126019097635 & 0.16148496966280 \\
\hline & $\mathrm{H}$ & 0.374873981397087 & 0.874873981594973 & 0.16148496966280 \\
\hline & $\mathrm{H}$ & 0.625126019097635 & 0.125126018899749 & \\
\hline & $\mathrm{H}$ & 0.874873981594973 & 0.374873981397087 & 0.16148496966280 \\
\hline & $\mathrm{H}$ & 0.122261339 & 0.877738660882329 & 0.2274238877190 \\
\hline & $\mathrm{H}$ & 0.374301611465952 & 0.625698389028770 & 0.22661943856190 \\
\hline & $\mathrm{H}$ & 0.625698389028770 & 0.374301611465952 & 0.2266194385619 \\
\hline
\end{tabular}




$\begin{array}{lllll}108 & \mathrm{H} & 0.877738660882329 & 0.122261339612393 & 0.227423887719076 \\ 109 & \mathrm{H} & 0.125005366205407 & 0.125005366205407 & 0.293031285747666 \\ 110 & \mathrm{H} & 0.374908323892945 & 0.374908323892945 & 0.290659540031455 \\ 111 & \mathrm{H} & 0.625091676601776 & 0.625091676601776 & 0.290659540031455 \\ 112 & \mathrm{H} & 0.874994634289315 & 0.874994634289315 & 0.293031285747666 \\ 113 & \mathrm{H} & 0.128408748346494 & 0.376294796574193 & 0.358007826632061 \\ 114 & \mathrm{H} & 0.376294796574193 & 0.128408748346494 & 0.358007826632061 \\ 115 & \mathrm{H} & 0.623705203920529 & 0.871591252148228 & 0.358007826632061 \\ 116 & \mathrm{H} & 0.871591252148228 & 0.623705203920529 & 0.358007826632061 \\ 117 & \mathrm{H} & 0.117783671342238 & 0.629679403378041 & 0.424873134510093 \\ 118 & \mathrm{H} & 0.370320597116681 & 0.882216329152484 & 0.424873134510093 \\ 119 & \mathrm{H} & 0.629679403378041 & 0.117783671342238 & 0.424873134510093 \\ 120 & \mathrm{H} & 0.882216329152484 & 0.370320597116681 & 0.424873134510093 \\ 121 & \mathrm{H} & 0.122690589958224 & 0.877309410536497 & 0.488054403187121 \\ 122 & \mathrm{H} & 0.877309410536497 & 0.122690589958224 & 0.488054403187121 \\ 123 & \mathrm{Ru} & 0.500000030079156 & 0.500000030079156 & 0.459296726102792 \\ 124 & \mathrm{Ru} & 0.305311875942088 & 0.478257299022963 & 0.501403127817965 \\ 125 & \mathrm{Ru} & 0.478257299022963 & 0.305311875942088 & 0.501403127817965 \\ 126 & \mathrm{Ru} & 0.521742761036399 & 0.694688124552634 & 0.501403127817965 \\ 127 & \mathrm{Ru} & 0.694688124552634 & 0.521742761036399 & 0.501403127817965\end{array}$

Table S7: Fractional atomic coordinates of the initial state of the $\mathrm{N}_{2}$ dissociation around $2 \mathrm{H}$ on $\mathrm{Ru} / \mathrm{Ca}_{2} \mathrm{NH}$. Lattice parameters are: $\mathrm{a}=\mathrm{b}=10.1067 \AA, \mathrm{c}=39.0701 \AA, \alpha=\beta=\gamma=$ 90 .

$\begin{array}{ccccc}\text { No. } & \text { Atom } & X & Y & Z \\ 1 & \mathrm{Ca} & 0.108186341016477 & 0.391813636918875 & 0.030294838468024 \\ 2 & \mathrm{Ca} & 0.391813636918875 & 0.108186341016477 & 0.030294838468024 \\ 3 & \mathrm{Ca} & 0.608186393358167 & 0.891813666998032 & 0.030294838468024 \\ 4 & \mathrm{Ca} & 0.891813666998032 & 0.608186393358167 & 0.030294838468024 \\ 5 & \mathrm{Ca} & 0.139695440095728 & 0.860304515676034 & 0.035014432581349 \\ 6 & \mathrm{Ca} & 0.360304545260469 & 0.639695485016574 & 0.035014432581349 \\ 7 & \mathrm{Ca} & 0.639695485016574 & 0.360304545260469 & 0.035014432581349 \\ 8 & \mathrm{Ca} & 0.860304515676034 & 0.139695440095728 & 0.035014432581349 \\ 9 & \mathrm{Ca} & 0.136710031423064 & 0.636710076343902 & 0.094508290935160 \\ 10 & \mathrm{Ca} & 0.363289953933140 & 0.863289924348706 & 0.094508290935160\end{array}$




\begin{tabular}{|c|c|c|c|c|}
\hline & $\mathrm{Ca}$ & & & \\
\hline & $\mathrm{Ca}$ & .863289924348706 & 0.363289953933140 & 0.094508290935160 \\
\hline & $\mathrm{Ca}$ & .113472400636908 & .113472400636908 & 0.099069771470752 \\
\hline & $\mathrm{Ca}$ & 577298444 & & 0.09906977 \\
\hline & $\mathrm{Ca}$ & 0.613472452978598 & 0.613472452978598 & 0.099069771470752 \\
\hline & $\mathrm{Ca}$ & & & 70752 \\
\hline & $\mathrm{Ca}$ & & & 0.15807191390794 \\
\hline & $\mathrm{Ca}$ & 0.387492224835007 & 0.612507805540978 & 1390794 \\
\hline & $\mathrm{Ca}$ & & & \\
\hline & $\mathrm{Ca}$ & 4164 & & 90794 \\
\hline & $\mathrm{Ca}$ & & & \\
\hline & $\mathrm{Ca}$ & 0.364175572493259 & 0.136496553044622 & 0.164568383166212 \\
\hline & $\mathrm{Ca}$ & 0.635824457783783 & 0.86350340 & 0.164568 \\
\hline & $\mathrm{Ca}$ & .863503402727147 & 0.635824457783783 & 0.1645 \\
\hline & $\mathrm{Ca}$ & 137095771781652 & 0.137095771781652 & 0.222 \\
\hline & & & & \\
\hline & $\mathrm{Ca}$ & 0.636063415883612 & 0.636063415883612 & 0.2227511 \\
\hline & $\mathrm{Ca}$ & & & \\
\hline & $\mathrm{Ca}$ & & & \\
\hline & $\mathrm{Ca}$ & 0.385235263960268 & 0.888145289079830 & 0.229247801103142 \\
\hline & $\mathrm{Ca}$ & & & 3142 \\
\hline & $\mathrm{Ca}$ & 0.888145289079830 & 0.385235263960268 & 0.229247801103142 \\
\hline & $\mathrm{Ca}$ & & & $418^{\circ}$ \\
\hline & & & & \\
\hline & $\mathrm{Ca}$ & & & \\
\hline & $\mathrm{Ca}$ & & & \\
\hline & $\mathrm{C}$ & & & \\
\hline & $\mathrm{Ca}$ & & 55506927607 & 0.2936503 \\
\hline & $\mathrm{Ca}$ & 0.641055506927607 & 0.358944523349443 & 0.293650308289748 \\
\hline & $\mathrm{Ca}$ & 0.867246693118808 & 0.132753262652961 & 0.29527812506432 \\
\hline & $\mathrm{Ca}$ & & & \\
\hline & $\mathrm{Ca}$ & 0.360660585915077 & 0.870855048239327 & 0.35224974464723 \\
\hline & $\mathrm{Ca}$ & 965 & 442 & 0.35 \\
\hline & $\mathrm{Ca}$ & 0.870855048239327 & 0.360660585915077 & 0.35224974464723 \\
\hline & $\mathrm{Ca}$ & 0.114742066798434 & 0.114742066798434 & 0.3641975303979 \\
\hline
\end{tabular}




\begin{tabular}{|c|c|c|c|c|}
\hline & $\mathrm{Ca}$ & & & \\
\hline & & .606880332692632 & 0.606880332692632 & 0.35787817008663 \\
\hline & $\mathrm{Ca}$ & 885257941315025 & 0.885257941315025 & 0.36419753039793 \\
\hline & & 106259876533897 & .893740131480612 & 0.42546676438550 \\
\hline & $\mathrm{Ca}$ & 338131093835500 & 0.661868936441543 & 0.41421402423140 \\
\hline & & & & \\
\hline & & 893740131480612 & & 0.42546676438550 \\
\hline & $\mathrm{Ca}$ & & & \\
\hline & & 380469762379100 & 0.151629614757951 & 0.42598752897976 \\
\hline & $\mathrm{Ca}$ & & & 897976 \\
\hline & & & 30267897943 & 0.42598752897976 \\
\hline & $\mathrm{Ca}$ & & & \\
\hline & & 1749 & 749 & 0.48996 \\
\hline & $\mathrm{Ca}$ & 597 & & \\
\hline & $\mathrm{Ca}$ & & & \\
\hline & & & & \\
\hline & $\mathrm{Ca}$ & & & \\
\hline & & & & 0.034742 \\
\hline & $\mathrm{N}$ & 0.378467634040553 & 0.878467634238447 & 0.034742 \\
\hline & & & & \\
\hline 66 & $\mathrm{~N}$ & 878467634238447 & 0.378467634040553 & 0.03474288158033 \\
\hline & $\mathrm{N}$ & & & 3961 \\
\hline & & 0.375549178205005 & 0.624450822289716 & 0.0963 \\
\hline & $\mathrm{N}$ & & 0.375 & 3961 \\
\hline & & & & 0.09 \\
\hline 71 & $\mathrm{~N}$ & 768 & & 849 \\
\hline 72 & $\mathrm{~N}$ & 0.374841609398060 & 0.374841609398060 & 0.16131582165049 \\
\hline 73 & $\mathrm{~N}$ & 0.625158391096661 & 0.625158391096661 & 0.16131582165049 \\
\hline 74 & $\mathrm{~N}$ & 0.874841609595954 & 0.874841609595954 & 0.16131582165049 \\
\hline 75 & $\mathrm{~N}$ & & & 0.2267983301 \\
\hline 76 & $\mathrm{~N}$ & 0.376116559683644 & 0.125709746018415 & 0.22679833016303 \\
\hline 77 & $\mathrm{~N}$ & 070 & 0.874 & 0.22679833016303 \\
\hline 78 & $\mathrm{~N}$ & 0.874290254476306 & 0.623883440811070 & 0.22679833016303 \\
\hline 79 & $\mathrm{~N}$ & 0.120674416167418 & 0.629635666249363 & 0.29156009786130 \\
\hline & $\mathrm{N}$ & 0.370364334245351 & 0.879325584327297 & 0.29156009786130 \\
\hline
\end{tabular}




\begin{tabular}{|c|c|c|c|c|}
\hline 81 & $\mathrm{~N}$ & 0.629635666249363 & 0.120674416167418 & 0.291560097861307 \\
\hline 82 & $\mathrm{~N}$ & 0.879325584327297 & 0.370364334245351 & 0.291560097861307 \\
\hline 83 & $\mathrm{~N}$ & 0.122881588482172 & 0.877118412012549 & 0.361385692857680 \\
\hline 84 & $\mathrm{~N}$ & .368625036263168 & 0.631374964231554 & 0.354363183333653 \\
\hline 85 & $\mathrm{~N}$ & 0.631374964231554 & 0.368625036263168 & 0.354363183333653 \\
\hline 86 & $\mathrm{~N}$ & 0.877118412012549 & 0.122881588482172 & 0.361385692857680 \\
\hline 87 & $\mathrm{~N}$ & 0.138663589231825 & 0.138663589231825 & 0.428655307516550 \\
\hline 88 & $\mathrm{~N}$ & 0.389487230618101 & 0.389487230618101 & 0.416209726989293 \\
\hline 89 & $\mathrm{~N}$ & 0.610512769876621 & 0.610512769876621 & 0.416209726989293 \\
\hline 90 & $\mathrm{~N}$ & 0.861336411262897 & 0.861336411262897 & 0.428655307516550 \\
\hline 91 & $\mathrm{~N}$ & 0.139723900832969 & 0.417784236685726 & 0.487409338286113 \\
\hline 92 & $\mathrm{~N}$ & 0.417784236685726 & 0.139723900832969 & 0.487409338286113 \\
\hline 93 & $\mathrm{~N}$ & 0.582215763808996 & 0.860276099661753 & 0.487409338286113 \\
\hline 94 & $\mathrm{~N}$ & 0.860276099661753 & 0.582215763808996 & 0.487409338286113 \\
\hline 95 & $\mathrm{~N}$ & 0.547049164147971 & 0.452950866129079 & 0.529616249438639 \\
\hline 96 & $\mathrm{~N}$ & 0.452950866129079 & 0.547049164147971 & 0.529616249438639 \\
\hline 97 & $\mathrm{H}$ & 0.133716503752943 & 0.133716503752943 & 0.032444094734203 \\
\hline 98 & $\mathrm{H}$ & 0.366283496543886 & 0.366283496543886 & 0.032444094734203 \\
\hline 99 & $\mathrm{H}$ & 0.633716503950836 & 0.633716503950836 & 0.032444094734203 \\
\hline 100 & $\mathrm{H}$ & 0.866283496741779 & 0.866283496741779 & 0.032444094734203 \\
\hline 101 & $\mathrm{H}$ & 0.125126018899749 & 0.625126019097635 & 0.161484969662801 \\
\hline 102 & $\mathrm{H}$ & 0.374873981397087 & 0.874873981594973 & 0.161484969662801 \\
\hline 103 & $\mathrm{H}$ & 0.625126019097635 & 0.125126018899749 & 0.161484969662801 \\
\hline 104 & $\mathrm{H}$ & 0.874873981594973 & 0.374873981397087 & 0.161484969662801 \\
\hline 105 & $\mathrm{H}$ & 0.121791435091310 & 0.878208565403412 & 0.228040573794388 \\
\hline 106 & $\mathrm{H}$ & 0.374823129679249 & 0.625176870815472 & 0.227270700922673 \\
\hline 107 & $\mathrm{H}$ & 0.625176870815472 & 0.374823129679249 & 0.227270700922673 \\
\hline 108 & $\mathrm{H}$ & 0.878208565403412 & 0.121791435091310 & 0.228040573794388 \\
\hline 109 & $\mathrm{H}$ & 0.124750055497045 & 0.124750055497045 & 0.293795400311374 \\
\hline 110 & $\mathrm{H}$ & 0.375555027145068 & 0.375555027145068 & 0.291753151527373 \\
\hline 111 & $\mathrm{H}$ & 0.624444973349653 & 0.624444973349653 & 0.291753151527373 \\
\hline 112 & $\mathrm{H}$ & 0.875249944997676 & 0.875249944997676 & 0.293795400311374 \\
\hline 113 & $\mathrm{H}$ & 0.130213514765102 & 0.375126416029310 & 0.358001073654115 \\
\hline 114 & $\mathrm{H}$ & 0.375126416029310 & 0.130213514765102 & 0.358001073654115 \\
\hline 115 & $\mathrm{H}$ & 0.624873584465412 & 0.869786485729619 & 0.358001073654115 \\
\hline
\end{tabular}




$\begin{array}{lllll}116 & \mathrm{H} & 0.869786485729619 & 0.624873584465412 & 0.358001073654115 \\ 117 & \mathrm{H} & 0.103083215669471 & 0.624729949740384 & 0.419998743653295 \\ 118 & \mathrm{H} & 0.375270050754338 & 0.896916784825251 & 0.419998743653295 \\ 119 & \mathrm{H} & 0.624729949740384 & 0.103083215669471 & 0.419998743653295 \\ 120 & \mathrm{H} & 0.896916784825251 & 0.375270050754338 & 0.419998743653295 \\ 121 & \mathrm{H} & 0.122919905563065 & 0.877080094931656 & 0.490072754978434 \\ 122 & \mathrm{H} & 0.877080094931656 & 0.122919905563065 & 0.490072754978434 \\ 123 & \mathrm{H} & 0.316521886491735 & 0.683478406582146 & 0.477553200818064 \\ 124 & \mathrm{H} & 0.683478406582146 & 0.316521886491735 & 0.477553200818064 \\ 125 & \mathrm{Ru} & 0.500000030079156 & 0.500000030079156 & 0.443892257666846 \\ 126 & \mathrm{Ru} & 0.321217819130815 & 0.490111408137920 & 0.492853566283216 \\ 127 & \mathrm{Ru} & 0.490111408137920 & 0.321217819130815 & 0.492853566283216 \\ 128 & \mathrm{Ru} & 0.509888651921450 & 0.678782181363907 & 0.492853566283216 \\ 129 & \mathrm{Ru} & 0.678782181363907 & 0.509888651921450 & 0.492853566283216\end{array}$

Table S8: Fractional atomic coordinates of the final state of the $\mathrm{N}_{2}$ dissociation around $2 \mathrm{H}$ on $\mathrm{Ru} / \mathrm{Ca}_{2} \mathrm{NH}$. Lattice parameters are: $\mathrm{a}=\mathrm{b}=10.1067 \AA, \mathrm{c}=39.0701 \AA, \alpha=\beta=\gamma=$ $90^{\circ}$.

$\begin{array}{ccccc}\text { No. } & \text { Atom } & X & Y & Z \\ 1 & \mathrm{Ca} & 0.108186341016477 & 0.391813636918875 & 0.030294838468024 \\ 2 & \mathrm{Ca} & 0.391813636918875 & 0.108186341016477 & 0.030294838468024 \\ 3 & \mathrm{Ca} & 0.608186393358167 & 0.891813666998032 & 0.030294838468024 \\ 4 & \mathrm{Ca} & 0.891813666998032 & 0.608186393358167 & 0.030294838468024 \\ 5 & \mathrm{Ca} & 0.139695440095728 & 0.860304515676034 & 0.035014432581349 \\ 6 & \mathrm{Ca} & 0.360304545260469 & 0.639695485016574 & 0.035014432581349 \\ 7 & \mathrm{Ca} & 0.639695485016574 & 0.360304545260469 & 0.035014432581349 \\ 8 & \mathrm{Ca} & 0.860304515676034 & 0.139695440095728 & 0.035014432581349 \\ 9 & \mathrm{Ca} & 0.136710031423064 & 0.636710076343902 & 0.094508290935160 \\ 10 & \mathrm{Ca} & 0.363289953933140 & 0.863289924348706 & 0.094508290935160 \\ 11 & \mathrm{Ca} & 0.636710076343902 & 0.136710031423064 & 0.094508290935160 \\ 12 & \mathrm{Ca} & 0.863289924348706 & 0.363289953933140 & 0.094508290935160 \\ 13 & \mathrm{Ca} & 0.113472400636908 & 0.113472400636908 & 0.099069771470752 \\ 14 & \mathrm{Ca} & 0.386527577298444 & 0.386527577298444 & 0.099069771470752 \\ 15 & \mathrm{Ca} & 0.613472452978598 & 0.613472452978598 & 0.099069771470752 \\ 16 & \mathrm{Ca} & 0.886527607377601 & 0.886527607377601 & 0.099069771470752\end{array}$




\begin{tabular}{|c|c|c|c|c|}
\hline & a & & & \\
\hline & & & & 0.15807191390794 \\
\hline & & 612507805540978 & .387492224835007 & 0.15807191390794 \\
\hline & $\mathrm{Ca}$ & & & \\
\hline & $\mathrm{Ca}$ & .137072151413690 & .362927833942507 & 0.16456455791317 \\
\hline & & & & \\
\hline & $\mathrm{Ca}$ & & & \\
\hline & & .862927804358079 & & 0.16 \\
\hline & $\mathrm{Ca}$ & & & \\
\hline & & & & 0.2 \\
\hline & $\mathrm{Ca}$ & 0.636399057376358 & & \\
\hline & & & & 0.22308692830952 \\
\hline & $\mathrm{Ca}$ & & & \\
\hline & & & & \\
\hline & $\mathrm{Ca}$ & 489002 & & \\
\hline & & & & \\
\hline & & & & \\
\hline & & & & \\
\hline & & & & \\
\hline & $\mathrm{Ca}$ & 0.885164065514423 & 0.612626028117702 & \\
\hline & & & & \\
\hline & $\mathrm{Ca}$ & 0.359088321517056 & & \\
\hline & & & & \\
\hline & & & & \\
\hline & & & & \\
\hline & & & & \\
\hline & & & & \\
\hline & & & & 0.3521 \\
\hline & $\mathrm{Ca}$ & & 0.115768164267152 & 0.3654921881 \\
\hline & & 0.387502283738456 & 0.387502283738456 & 0.35621069837515 \\
\hline & & & & \\
\hline & & 0.884231843846308 & 0.884231843846308 & 0.36549218812208 \\
\hline & & 124 & 0.8891860867 & 0.42474720968 \\
\hline & $\mathrm{Ca}$ & 0.373717372446698 & 0.626282657830344 & 0.4094448495230 \\
\hline & & 0.626282657830344 & 0.373717372446698 & 0.4094448495230 \\
\hline
\end{tabular}




\begin{tabular}{|c|c|c|c|c|}
\hline & $\mathrm{Ca}$ & .889186086754385 & & \\
\hline & $\mathrm{Ca}$ & 0.161600689648345 & & 0.43113508054813 \\
\hline & $\mathrm{Ca}$ & 381804351299287 & 0.161600689648345 & 0.43113508054813 \\
\hline & & & & \\
\hline & $\mathrm{Ca}$ & 838399266123417 & 0.618195678977763 & 0.43113508054813 \\
\hline & $\mathrm{Ca}$ & & & 984569 \\
\hline & $\mathrm{Ca}$ & 828740759205871 & 0.828740759205871 & 0.49269141984569 \\
\hline & $\mathrm{Ca}$ & & & 1240 \\
\hline & $\mathrm{Ca}$ & .362084781476397 & 0.908921354259739 & 0.49387900951240 \\
\hline & $\mathrm{Ca}$ & & & \\
\hline & $\mathrm{Ca}$ & 908921354259739 & 0.362084781476397 & 0.49387900951240 \\
\hline & $\mathrm{N}$ & & & 0.034 \\
\hline & $\mathrm{N}$ & 378467634040553 & 0.878467634238447 & 0.0347428 \\
\hline & $\mathrm{N}$ & & & 0.03474 \\
\hline & $\mathrm{N}$ & & & \\
\hline & & 24450822091830 & 91 & \\
\hline & $\mathrm{N}$ & 8205005 & & \\
\hline & & 9716 & 205005 & 0.09632 \\
\hline 70 & $\mathrm{~N}$ & 0.875549178402891 & 0.124450822091830 & 0.09632835963961 \\
\hline & $\mathrm{N}$ & & & 049 \\
\hline 72 & $\mathrm{~N}$ & 374841609398060 & 0.374841609398060 & 0.16131582165049 \\
\hline & $\mathrm{N}$ & & & 5049 \\
\hline & $\mathrm{N}$ & 874841609595954 & 0.874841609595954 & 0.16131582165049 \\
\hline & $\mathrm{N}$ & 109 & 0.3759 & 0.22 \\
\hline & $\mathrm{N}$ & 0.375970873777305 & & \\
\hline 77 & $\mathrm{~N}$ & 417 & 0.87 & 0.226 \\
\hline 78 & $\mathrm{~N}$ & 0.874221841153612 & 0.624029126717417 & 0.22643164076043 \\
\hline 79 & $\mathrm{~N}$ & 0.120197004406414 & 0.629897060849259 & 0.29121416042929 \\
\hline 80 & $\mathrm{~N}$ & 0.370102939645462 & 0.879802996088315 & 0.29121416042929 \\
\hline & $\mathrm{N}$ & & & 0.29121 \\
\hline 82 & $\mathrm{~N}$ & 0.879802996088315 & 0.370102939645462 & 0.29121416042929 \\
\hline & $\mathrm{N}$ & 73936936851 & 0.877026063557870 & 1613110751 \\
\hline 84 & $\mathrm{~N}$ & 0.372499574630133 & 0.627500425864589 & 0.35109050538626 \\
\hline (j) & $\mathrm{N}$ & 0.627500425864589 & 0.372499574630133 & 0.35109050538626 \\
\hline & $\mathrm{N}$ & 0.877026063557870 & 0.122973936936851 & 0.36121613110751 \\
\hline
\end{tabular}




$\begin{array}{ccccc}87 & \mathrm{~N} & 0.137166064997657 & 0.137166064997657 & 0.430467032487143 \\ 88 & \mathrm{~N} & 0.390546150507425 & 0.390546150507425 & 0.417116510152503 \\ 89 & \mathrm{~N} & 0.609453849987296 & 0.609453849987296 & 0.417116510152503 \\ 90 & \mathrm{~N} & 0.862833935497064 & 0.862833935497064 & 0.430467032487143 \\ 91 & \mathrm{~N} & 0.135894918854724 & 0.402290946527280 & 0.493549342565827 \\ 92 & \mathrm{~N} & 0.402290946527280 & 0.135894918854724 & 0.493549342565827 \\ 93 & \mathrm{~N} & 0.597709053967442 & 0.864105081639998 & 0.493549342565827 \\ 94 & \mathrm{~N} & 0.864105081639998 & 0.597709053967442 & 0.493549342565827 \\ 95 & \mathrm{~N} & 0.345871253072004 & 0.654128777303988 & 0.517339152739509 \\ 96 & \mathrm{~N} & 0.654128777303988 & 0.345871253072004 & 0.517339152739509 \\ 97 & \mathrm{H} & 0.133716503752943 & 0.133716503752943 & 0.032444094734203 \\ 98 & \mathrm{H} & 0.366283496543886 & 0.366283496543886 & 0.032444094734203 \\ 99 & \mathrm{H} & 0.633716503950836 & 0.633716503950836 & 0.032444094734203 \\ 100 & \mathrm{H} & 0.866283496741779 & 0.866283496741779 & 0.032444094734203 \\ 101 & \mathrm{H} & 0.125126018899749 & 0.625126019097635 & 0.161484969662801 \\ 102 & \mathrm{H} & 0.374873981397087 & 0.874873981594973 & 0.161484969662801 \\ 103 & \mathrm{H} & 0.625126019097635 & 0.125126018899749 & 0.161484969662801 \\ 104 & \mathrm{H} & 0.874873981594973 & 0.374873981397087 & 0.161484969662801 \\ 105 & \mathrm{H} & 0.122485616853978 & 0.877514383640743 & 0.227140463514096 \\ 106 & \mathrm{H} & 0.374333125664506 & 0.625666874830216 & 0.226274939043300 \\ 107 & \mathrm{H} & 0.625666874830216 & 0.374333125664506 & 0.226274939043300 \\ 108 & \mathrm{H} & 0.877514383640743 & 0.122485616853978 & 0.227140463514096 \\ 1116 & \mathrm{H} & 0.124063614126562 & 0.124063614126562 & 0.292445950641557 \\ 110 & \mathrm{H} & 0.373645749631698 & 0.373645749631698 & 0.290120101027866 \\ 111 & \mathrm{H} & 0.626354250863023 & 0.626354250863023 & 0.290120101027866 \\ 112 & \mathrm{H} & 0.875936386368160 & 0.875936386368160 & 0.292445950641557 \\ \mathrm{H} & \mathrm{H} & 0.127989140738229 & 0.375388294824155 & 0.357045074476033 \\ 114 & 0.375388294824155 & 0.127989140738229 & 0.357045074476033 \\ 115 & 0.624611705670567 & 0.872010859756493 & 0.357045074476033 \\ 10.8726511380417589 & 0.873488620077132 & 0.489040658187285\end{array}$




$\begin{array}{lllll}122 & \mathrm{H} & 0.873488620077132 & 0.126511380417589 & 0.489040658187285 \\ 123 & \mathrm{H} & 0.383448117515790 & 0.616552175558091 & 0.462506458968569 \\ 124 & \mathrm{H} & 0.616552175558091 & 0.383448117515790 & 0.462506458968569 \\ 125 & \mathrm{Ru} & 0.500000030079156 & 0.500000030079156 & 0.463652296970330 \\ 126 & \mathrm{Ru} & 0.303970034712797 & 0.480431526756483 & 0.506668072311292 \\ 127 & \mathrm{Ru} & 0.480431526756483 & 0.303970034712797 & 0.506668072311292 \\ 128 & \mathrm{Ru} & 0.519568533302880 & 0.696029965781924 & 0.506668072311292 \\ 129 & \mathrm{Ru} & 0.696029965781924 & 0.519568533302880 & 0.506668072311292\end{array}$

Table S9: Fractional atomic coordinates of the initial state of the $\mathrm{N}_{2}$ dissociation on $\mathrm{Ru} / \mathrm{Ca}_{2} \mathrm{NH}\left(E_{\mathrm{a}}=1.06 \mathrm{eV}\right)$. Lattice parameters are: $\mathrm{a}=\mathrm{b}=10.1067 \AA, \mathrm{c}=25.7530 \AA, \alpha$ $=\beta=\gamma=90^{\circ}$.

$\begin{array}{ccccc}\text { No. } & \text { Atom } & X & Y & Z \\ 1 & \mathrm{Ca} & 0.386888057183512 & 0.386888057183512 & 0.151832714655498 \\ 2 & \mathrm{Ca} & 0.859843686508818 & 0.859843686508818 & 0.347493567914096 \\ 3 & \mathrm{Ca} & 0.625151230254004 & 0.112531518427942 & 0.359247051832615 \\ 4 & \mathrm{Ca} & 0.636888087064782 & 0.136888042143937 & 0.142501875733735 \\ 5 & \mathrm{Ca} & 0.112620668128333 & 0.887156863782488 & 0.244928471660551 \\ 6 & \mathrm{Ca} & 0.132868315361505 & 0.362348331134014 & 0.250523851143242 \\ 7 & \mathrm{Ca} & 0.886888087262669 & 0.613111973093531 & 0.044390339404067 \\ 8 & \mathrm{Ca} & 0.363111943212260 & 0.636888087064782 & 0.053721185781271 \\ 9 & \mathrm{Ca} & 0.136888042143937 & 0.636888087064782 & 0.142501875733735 \\ 10 & \mathrm{Ca} & 0.112531518427942 & 0.625151230254004 & 0.359247051832615 \\ 11 & \mathrm{Ca} & 0.636888087064782 & 0.363111943212260 & 0.053721185781271 \\ 12 & \mathrm{Ca} & 0.613111973093531 & 0.886888087262669 & 0.044390339404067 \\ 13 & \mathrm{Ca} & 0.362348331134014 & 0.132868315361505 & 0.250523851143242 \\ 14 & \mathrm{Ca} & 0.887156863782488 & 0.112620668128333 & 0.244928471660551 \\ 15 & \mathrm{Ca} & 0.381081005299890 & 0.890204961909852 & 0.369347609093908 \\ 16 & \mathrm{Ca} & 0.863111913627832 & 0.363111943212260 & 0.142501875733735 \\ 17 & \mathrm{Ca} & 0.613111973093531 & 0.613111973093531 & 0.151832714655498 \\ 18 & \mathrm{Ca} & 0.620669714576500 & 0.620669714576500 & 0.330949527482592 \\ 19 & \mathrm{Ca} & 0.113111920751841 & 0.386888057183512 & 0.044390339404067 \\ 20 & \mathrm{Ca} & 0.136888042143937 & 0.863111913627832 & 0.053721185781271 \\ 21 & \mathrm{Ca} & 0.139841035430273 & 0.139841035430273 & 0.347936633974061 \\ 22 & \mathrm{Ca} & 0.113111920751841 & 0.113111920751841 & 0.151832714655498\end{array}$




\begin{tabular}{|c|c|c|c|c|}
\hline & $\mathrm{Ca}$ & & & \\
\hline & $\mathrm{Ca}$ & .618930313152995 & & 0.23794423009360 \\
\hline & $\mathrm{Ca}$ & 890204961909852 & 0.381081005299890 & 0.36934760909390 \\
\hline & $\mathrm{Ca}$ & & & \\
\hline & $\mathrm{Ca}$ & .383748178004266 & 0.618930313152995 & 0.23794423009360 \\
\hline & $\mathrm{Ca}$ & 613546725 & & 0.253 \\
\hline & $\mathrm{Ca}$ & & & 0.05372118578127 \\
\hline & $\mathrm{Ca}$ & 0.386888057183512 & 0.113111920751841 & 0.04439033 \\
\hline & $\mathrm{Ca}$ & & & \\
\hline & & & & \\
\hline & $\mathrm{N}$ & 0.625337109469566 & & 0.241 \\
\hline & $\mathrm{N}$ & & & 0.04905576630097 \\
\hline & $\mathrm{N}$ & 0.87500000039 & 0.3750000001 & 0.0490 \\
\hline & $\mathrm{N}$ & 0.875000000395779 & & 0.1471672 \\
\hline & $\mathrm{N}$ & 531165547827 & 39083363494 & 0.357 \\
\hline & $\mathrm{N}$ & & & \\
\hline & $\mathrm{N}$ & & 0.875000000395779 & \\
\hline & $\mathrm{N}$ & & & \\
\hline & & & & \\
\hline & $\mathrm{N}$ & 0.873519473202526 & 0.873519473202526 & 0.25148174708675 \\
\hline & $\mathrm{N}$ & & & 0.350 \\
\hline 44 & $\mathrm{~N}$ & 0.375000000197886 & 0.625000000296836 & 0.147167295214032 \\
\hline & $\mathrm{N}$ & & 6836 & 097 \\
\hline & $\mathrm{N}$ & & & \\
\hline & $\mathrm{N}$ & & & \\
\hline & $\mathrm{N}$ & & & 0.2528 \\
\hline & $\mathrm{N}$ & & 0.63 & \\
\hline & $\mathrm{N}$ & 0.724097629115777 & 0.724097629115777 & 0.48676095518174 \\
\hline 51 & $\mathrm{H}$ & 0.125000000098943 & 0.125000000098943 & 0.049055766300974 \\
\hline & $\mathrm{H}$ & 0.888378315452834 & 0.383009776995750 & 0.23995611229345 \\
\hline & $\mathrm{H}$ & & & \\
\hline & $\mathrm{H}$ & 0.358719907317960 & 0.654020038322688 & 0.34392561154211 \\
\hline & $\mathrm{H}$ & 395779 & 296836 & 0.1 \\
\hline 0 & $\mathrm{H}$ & 0.625000000296836 & 0.875000000395779 & 0.147167295214032 \\
\hline & $\mathrm{H}$ & 0.654020038322688 & 0.358719907317960 & 0.34392561154211 \\
\hline
\end{tabular}




$\begin{array}{lllll}58 & \mathrm{H} & 0.121632536583579 & 0.622066690136150 & 0.251987498156839 \\ 59 & \mathrm{H} & 0.875000000395779 & 0.875000000395779 & 0.049055766300974 \\ 60 & \mathrm{H} & 0.375000000197886 & 0.375000000197886 & 0.049055766300974 \\ 61 & \mathrm{H} & 0.375000000197886 & 0.125000000098943 & 0.147167295214032 \\ 62 & \mathrm{H} & 0.879025659898075 & 0.122104772060622 & 0.346722267426820 \\ 63 & \mathrm{H} & 0.622066690136150 & 0.121632536583579 & 0.251987498156839 \\ 64 & \mathrm{H} & 0.122104772060622 & 0.879025659898075 & 0.346722267426820 \\ 65 & \mathrm{H} & 0.125000000098943 & 0.375000000197886 & 0.147167295214032 \\ 66 & \mathrm{H} & 0.625000000296836 & 0.625000000296836 & 0.049055766300974 \\ 67 & \mathrm{Ru} & 0.410288593083348 & 0.580158163123427 & 0.403788718347707 \\ 68 & \mathrm{Ru} & 0.580158163123427 & 0.410288593083348 & 0.403788718347707 \\ 69 & \mathrm{Ru} & 0.612864659040189 & 0.779473848212697 & 0.417781166677882 \\ 70 & \mathrm{Ru} & 0.779473848212697 & 0.612864659040189 & 0.417781166677882 \\ 71 & \mathrm{Ru} & 0.244510259212726 & 0.408109504159268 & 0.417347821259975 \\ 72 & \mathrm{Ru} & 0.408109504159268 & 0.244510259212726 & 0.417347821259975\end{array}$

Table S10: Fractional atomic coordinates of the final state of the $\mathrm{N}_{2}$ dissociation on $\mathrm{Ru} / \mathrm{Ca}_{2} \mathrm{NH}\left(E_{\mathrm{a}}=1.06 \mathrm{eV}\right)$. Lattice parameters are: $\mathrm{a}=\mathrm{b}=10.1067 \AA, \mathrm{c}=25.7530 \AA, \alpha$ $=\beta=\gamma=90$ 。.

$\begin{array}{ccccc}\text { No. } & \text { Atom } & X & Y & Z \\ 1 & \mathrm{Ca} & 0.386888057183512 & 0.386888057183512 & 0.151832714655498 \\ 2 & \mathrm{Ca} & 0.853616010426469 & 0.853616010426469 & 0.347162788720517 \\ 3 & \mathrm{Ca} & 0.627750863794906 & 0.095696817392051 & 0.368872653817111 \\ 4 & \mathrm{Ca} & 0.636888087064782 & 0.136888042143937 & 0.142501875733735 \\ 5 & \mathrm{Ca} & 0.110197968653066 & 0.885374093374537 & 0.244353097655943 \\ 6 & \mathrm{Ca} & 0.135103697468042 & 0.363648350996740 & 0.250744476641180 \\ 7 & \mathrm{Ca} & 0.886888087262669 & 0.613111973093531 & 0.044390339404067 \\ 8 & \mathrm{Ca} & 0.363111943212260 & 0.636888087064782 & 0.053721185781271 \\ 9 & \mathrm{Ca} & 0.136888042143937 & 0.636888087064782 & 0.142501875733735 \\ 10 & \mathrm{Ca} & 0.095696817392051 & 0.627750863794906 & 0.368872653817111 \\ 11 & \mathrm{Ca} & 0.636888087064782 & 0.363111943212260 & 0.053721185781271 \\ 12 & \mathrm{Ca} & 0.613111973093531 & 0.886888087262669 & 0.044390339404067 \\ 13 & \mathrm{Ca} & 0.363648350996740 & 0.135103697468042 & 0.250744476641180 \\ 14 & \mathrm{Ca} & 0.885374093374537 & 0.110197968653066 & 0.244353097655943 \\ 15 & \mathrm{Ca} & 0.377226742756522 & 0.894173932657072 & 0.360922037723548\end{array}$




\begin{tabular}{|c|c|c|c|c|}
\hline & $\mathrm{Ca}$ & 863111913627832 & & \\
\hline & & 0.613111973093531 & 0.613111973093531 & 0.15183271465549 \\
\hline & $\mathrm{Ca}$ & .629234303272099 & 0.629234303272099 & 0.33190264665859 \\
\hline & $\mathrm{Ca}$ & .113111920751841 & .386888057183512 & 0.04439033940406 \\
\hline & $\mathrm{Ca}$ & 0.136888042143937 & 0.863111913627832 & 0.05372118578127 \\
\hline & $\mathrm{Ca}$ & & & \\
\hline & $\mathrm{Ca}$ & .113111920751841 & 0.113111920751841 & 0.15183271465549 \\
\hline & $\mathrm{Ca}$ & & & \\
\hline & $\mathrm{Ca}$ & 0.618359465278009 & 0.384024902990424 & 0.23767152997078 \\
\hline & $\mathrm{Ca}$ & & & \\
\hline & $\mathrm{Ca}$ & 2260 & 13627832 & 0.14250187573373 \\
\hline & $\mathrm{Ca}$ & & & \\
\hline & & 610486 & & 0.25222681968547 \\
\hline & $\mathrm{Ca}$ & & & \\
\hline & $\mathrm{Ca}$ & & & \\
\hline & & & & \\
\hline & $\mathrm{Ca}$ & & & \\
\hline & $\mathrm{N}$ & 0.62206 & 030 & 0.241 \\
\hline & $\mathrm{N}$ & 0.375000000197886 & 0.875000000395779 & 0.04905576630097 \\
\hline & & & & \\
\hline & $\mathrm{N}$ & 0.875000000395779 & 0.125000000098943 & 0.1471672 \\
\hline & $\mathrm{N}$ & & & 0 . \\
\hline & $\mathrm{N}$ & & 0.380974 & 0.357426 \\
\hline & $\mathrm{N}$ & & 0.87500000 & 19.17. \\
\hline & $\mathrm{N}$ & & & \\
\hline & $\mathrm{N}$ & & 7065 & 00 \\
\hline & $\mathrm{N}$ & 0.872885946211499 & 0.872885946211499 & 0.24874992380622 \\
\hline & $\mathrm{N}$ & 0.874191726658304 & 0.606841780492536 & 0.3432563823977 \\
\hline & $\mathrm{N}$ & 0.375000000197886 & 0.625000000296836 & 0.14716729521403 \\
\hline & $\mathrm{N}$ & & & 0.0490557663 \\
\hline & $\mathrm{N}$ & 0.625000000296836 & 0.375000000197886 & 0.14716729521403 \\
\hline & $\mathrm{N}$ & & & \\
\hline & $\mathrm{N}$ & 0.127995554976052 & 0.127995554976052 & 0.25296568195140 \\
\hline & $\mathrm{N}$ & 0.774900473361252 & 0.774900473361252 & 0.4284431225832 \\
\hline & $\mathrm{N}$ & 0.580475559476945 & 0.580475559476945 & 0.4392647852248 \\
\hline
\end{tabular}




$\begin{array}{lllll}51 & \mathrm{H} & 0.125000000098943 & 0.125000000098943 & 0.049055766300974 \\ 52 & \mathrm{H} & 0.881940434752417 & 0.372047057659753 & 0.247075411405525 \\ 53 & \mathrm{H} & 0.372047057659753 & 0.881940434752417 & 0.247075411405525 \\ 54 & \mathrm{H} & 0.325176971922558 & 0.668079876120993 & 0.354797582841762 \\ 55 & \mathrm{H} & 0.875000000395779 & 0.625000000296836 & 0.147167295214032 \\ 56 & \mathrm{H} & 0.625000000296836 & 0.875000000395779 & 0.147167295214032 \\ 57 & \mathrm{H} & 0.668079876120993 & 0.325176971922558 & 0.354797582841762 \\ 58 & \mathrm{H} & 0.121540062838795 & 0.618631844025211 & 0.244071816172917 \\ 59 & \mathrm{H} & 0.875000000395779 & 0.875000000395779 & 0.049055766300974 \\ 60 & \mathrm{H} & 0.375000000197886 & 0.375000000197886 & 0.049055766300974 \\ 61 & \mathrm{H} & 0.375000000197886 & 0.125000000098943 & 0.147167295214032 \\ 62 & \mathrm{H} & 0.877571106085610 & 0.120452804269680 & 0.344979320322098 \\ 63 & \mathrm{H} & 0.618631844025211 & 0.121540062838795 & 0.244071816172917 \\ 64 & \mathrm{H} & 0.120452804269680 & 0.877571106085610 & 0.344979320322098 \\ 65 & \mathrm{H} & 0.125000000098943 & 0.375000000197886 & 0.147167295214032 \\ 66 & \mathrm{H} & 0.625000000296836 & 0.625000000296836 & 0.049055766300974 \\ 67 & \mathrm{Ru} & 0.408216768188841 & 0.589174802811634 & 0.405313486661213 \\ 68 & \mathrm{Ru} & 0.589174802811634 & 0.408216768188841 & 0.405313486661213 \\ 69 & \mathrm{Ru} & 0.598462975762477 & 0.792210320178488 & 0.419021764035713 \\ 70 & \mathrm{Ru} & 0.792210320178488 & 0.598462975762477 & 0.419021764035713 \\ 71 & \mathrm{Ru} & 0.244200405803047 & 0.411275301777326 & 0.416409924953442 \\ 72 & \mathrm{Ru} & 0.411275301777326 & 0.244200405803047 & 0.416409924953442\end{array}$

Table S11: Fractional atomic coordinates of the initial state of the $\mathrm{NH}$ formation on $\mathrm{Ru} / \mathrm{Ca}_{2} \mathrm{NH}$. Lattice parameters are: $\mathrm{a}=\mathrm{b}=10.1067 \AA, \mathrm{c}=39.0701 \AA, \alpha=\beta=\gamma=90^{\circ}$. No. Atom $X$

$\begin{array}{lll}1 & \mathrm{Ca} & 0.108115437024963 \\ 2 & \mathrm{Ca} & 0.391911555158699 \\ 3 & \mathrm{Ca} & 0.608088474821514 \\ 4 & \mathrm{Ca} & 0.891884570890603 \\ 5 & \mathrm{Ca} & 0.139734689832295 \\ 6 & \mathrm{Ca} & 0.360208036288476 \\ 7 & \mathrm{Ca} & 0.639791993691737 \\ 8 & \mathrm{Ca} & 0.860265266137361 \\ 9 & \mathrm{Ca} & 0.136610523539424\end{array}$

0.391911555158699

0.030150181941693

0.108115437024963

0.030150181941693

0.891884570890603

0.030150181941693

0.608088474821514

0.030150181941693

0.860265266137361

0.034964078332301

0.639791993691737

0.034944222405848

0.360208036288476

0.034944222405848

0.139734689832295

0.034964078332301

0.636753359259927

0.094502413941925 


\begin{tabular}{|c|c|c|c|c|}
\hline & $\mathrm{a}$ & & & \\
\hline & $\mathrm{Ca}$ & 636753359259927 & 0.136610523539424 & 0.09450241394192 \\
\hline & & 863389431440786 & 0.363246670819222 & 0.09450241394192 \\
\hline & $\mathrm{Ca}$ & & 7904 & 0.098 \\
\hline & $\mathrm{Ca}$ & .386583350682940 & 0.386583350682940 & 0.09891037678706 \\
\hline & $\mathrm{Ca}$ & & & 0.09 \\
\hline & $\mathrm{Ca}$ & & & \\
\hline & $\mathrm{Ca}$ & .112350651944332 & 0.887649356070177 & 0.157983 \\
\hline & $\mathrm{Ca}$ & & & \\
\hline & $\mathrm{Ca}$ & & & 0.1 \\
\hline & $\mathrm{Ca}$ & 0.887649356070177 & & \\
\hline & $\mathrm{Ca}$ & 0.137486222954997 & 0.363227812474697 & 0.164436050903042 \\
\hline & $\mathrm{Ca}$ & 3227812474697 & 2954997 & 0.16443605090304 \\
\hline & $\mathrm{Ca}$ & 17505509 & & \\
\hline & $\mathrm{Ca}$ & 2025213 & 7505509 & 0.164 \\
\hline & $\mathrm{Ca}$ & & & \\
\hline & $\mathrm{Ca}$ & 0.363882091387857 & 0.363575521972514 & \\
\hline & $\mathrm{Ca}$ & & & \\
\hline & $\mathrm{Ca}$ & & & \\
\hline & $\mathrm{Ca}$ & 0.112820113226007 & 0.615145996035238 & 0.2294436777678 \\
\hline & $\mathrm{Ca}$ & & & \\
\hline & $\mathrm{Ca}$ & & & 0.22879871618160 \\
\hline & $\mathrm{Ca}$ & 07425 & & \\
\hline & & & & \\
\hline & $\mathrm{Ca}$ & & & \\
\hline & $\mathrm{Ca}$ & & & \\
\hline & $\mathrm{Ca}$ & & & \\
\hline & $\mathrm{Ca}$ & & 0.866224045731272 & 0.296149532206079 \\
\hline & $\mathrm{Ca}$ & 0.359889140024520 & 0.640110889955686 & 0.2940762233796 \\
\hline & $\mathrm{Ca}$ & 0.641139951827121 & 0.358860078153085 & 0.290916030074733 \\
\hline & $\mathrm{Ca}$ & 0.868070964090812 & & \\
\hline & $\mathrm{Ca}$ & 0.133113934399311 & 0.639466480835949 & 0.35384924090654 \\
\hline & $\mathrm{Ca}$ & 257 & 30899 & 0.35 \\
\hline & $\mathrm{Ca}$ & 0.637538278139167 & 0.130888626957343 & 0.35076145196 \\
\hline & $\mathrm{Ca}$ & 0.869111328022867 & 0.362461751841038 & 0.350761451965 \\
\hline
\end{tabular}




\begin{tabular}{|c|c|c|c|c|}
\hline & $\mathrm{Ca}$ & & & \\
\hline & & .389016484478887 & .389311384455872 & .35682127714493 \\
\hline & $\mathrm{Ca}$ & .610688646513786 & 0.610983546490771 & O 25602107714402 \\
\hline & & & & \\
\hline & $\mathrm{Ca}$ & 111444895543513 & 0.888555112470996 & 12077100401625 \\
\hline & $\mathrm{Ca}$ & & & 507678 \\
\hline & & 635725095080509 & 0.364274934998647 & 040601425100045 \\
\hline & $\mathrm{Ca}$ & & & \\
\hline & $\mathrm{Ca}$ & .160921250635468 & 0.379227231754057 & 0.42730225319802 \\
\hline & $\mathrm{Ca}$ & & & 077 \\
\hline & $\mathrm{Ca}$ & & & 0 \\
\hline & $\mathrm{Ca}$ & & & 977 \\
\hline & $\mathrm{Ca}$ & 166402296244982 & 0.176146144977224 & (10042702706107 \\
\hline & $\mathrm{Ca}$ & 0.823853810992439 & 0.833597659724674 & 706107 \\
\hline & $\mathrm{Ca}$ & 000 & & 110 \\
\hline & & .356978414878284 & & 38274467110 \\
\hline & $\mathrm{Ca}$ & & & \\
\hline & $\mathrm{Ca}$ & .905319771267727 & & 0.48 \\
\hline & $\mathrm{N}$ & 0.121479989738574 & 0.621553502800040 & 0.03480350986244 \\
\hline & $\mathrm{N}$ & & & 624 \\
\hline 65 & $\mathrm{~N}$ & 621553502800040 & 0.121479989738574 & 0.0348035 \\
\hline & $\mathrm{N}$ & & & $4=$ \\
\hline & $\mathrm{N}$ & 0.124247063514481 & 0.875752936485518 & 0.096 \\
\hline & $\mathrm{N}$ & 0.375 & & 134 \\
\hline & $\mathrm{N}$ & & & 75754 \\
\hline 70 & $\mathrm{~N}$ & & & 0 \\
\hline 71 & $\mathrm{~N}$ & 0.125408497299822 & 0.125408497299822 & 0.16140867353657 \\
\hline 72 & $\mathrm{~N}$ & 0.374956399867613 & 0.374956399867613 & 10000805030 \\
\hline 72 & $\mathrm{~N}$ & 0.625043600132386 & 0.625043600132386 & 016116066805626 \\
\hline 74 & $\mathrm{~N}$ & & & 0.1614086735365 \\
\hline 75 & $\mathrm{~N}$ & 0.126942468015236 & 0.376164425262913 & 0.22608962004129 \\
\hline 76 & $\mathrm{~N}$ & 0.375782270850415 & 1190660470 & 139380 \\
\hline 77 & $\mathrm{~N}$ & 0.623835574737086 & 0.873057531984763 & 0.22608962004129 \\
\hline 78 & $\mathrm{~N}$ & 0.874818809339529 & 0.624217729149585 & 0.22627880139380 \\
\hline & $\mathrm{N}$ & 0.121969390162583 & 0.628048158105166 & 0.2917098620704 \\
\hline
\end{tabular}




\begin{tabular}{|c|c|c|c|c|}
\hline 80 & $\mathrm{~N}$ & 0.371951842884279 & 0.878030609837416 & 0.291709862070405 \\
\hline 81 & $\mathrm{~N}$ & 0.630547481241357 & 0.118673652797774 & 0.290377922668575 \\
\hline 82 & $\mathrm{~N}$ & 0.881326347202225 & 0.369452519748087 & 0.290377922668575 \\
\hline 83 & $\mathrm{~N}$ & 0.121195400618830 & 0.878804599381169 & 0.360467113708935 \\
\hline 84 & $\mathrm{~N}$ & 0.370438901761616 & 0.629561099227828 & 0.354572771342567 \\
\hline 85 & $\mathrm{~N}$ & 0.628058563748588 & 0.371941437240856 & 0.349168041426899 \\
\hline 86 & $\mathrm{~N}$ & 0.875465894083291 & 0.124534105916708 & 0.360483346444311 \\
\hline 87 & $\mathrm{~N}$ & 0.136604419816365 & 0.136269235769738 & 0.429003683320907 \\
\hline 88 & $\mathrm{~N}$ & 0.401430403015737 & 0.382098970533128 & 0.416479078636818 \\
\hline 89 & $\mathrm{~N}$ & 0.617901029466871 & 0.598569596984262 & 0.416479078636818 \\
\hline 90 & $\mathrm{~N}$ & 0.863730765219706 & 0.863395581173080 & 0.429003683320907 \\
\hline 91 & $\mathrm{~N}$ & 0.133142465250244 & 0.408927626059068 & 0.488127379362460 \\
\hline 92 & $\mathrm{~N}$ & 0.396903931840292 & 0.134037756736667 & 0.494240916894781 \\
\hline 93 & $\mathrm{~N}$ & 0.591072373940932 & 0.866857535739200 & 0.488127379362460 \\
\hline 94 & $\mathrm{~N}$ & 0.865962244252777 & 0.603096068159708 & 0.494240916894781 \\
\hline 95 & $\mathrm{~N}$ & 0.335052437880385 & 0.664947592198771 & 0.494782110848653 \\
\hline 96 & $\mathrm{~N}$ & 0.618611246502574 & 0.381388783576582 & 0.523586638242065 \\
\hline 97 & $\mathrm{H}$ & 0.133723336371751 & 0.133723336371751 & 0.032621928917692 \\
\hline 98 & $\mathrm{H}$ & 0.366273334736391 & 0.366273334736391 & 0.032601939743728 \\
\hline 99 & $\mathrm{H}$ & 0.633726666253053 & 0.633726666253053 & 0.032601939743728 \\
\hline 100 & $\mathrm{H}$ & 0.866276664617693 & 0.866276664617693 & 0.032621928917692 \\
\hline 101 & $\mathrm{H}$ & 0.124749278064634 & 0.625437115934694 & 0.161574204132399 \\
\hline 102 & $\mathrm{H}$ & 0.374562884065305 & 0.875250721935366 & 0.161574204132399 \\
\hline 103 & $\mathrm{H}$ & 0.625437115934694 & 0.124749278064634 & 0.161574204132399 \\
\hline 104 & $\mathrm{H}$ & 0.875250721935366 & 0.374562884065305 & 0.161574204132399 \\
\hline 105 & $\mathrm{H}$ & 0.123568684394790 & 0.876431315605209 & 0.226784178468314 \\
\hline 106 & $\mathrm{H}$ & 0.374330268615139 & 0.625669732374305 & 0.226285344556437 \\
\hline 107 & $\mathrm{H}$ & 0.626270840306759 & 0.373729160682685 & 0.225423814868243 \\
\hline 108 & $\mathrm{H}$ & 0.877883697677603 & 0.122116302322396 & 0.226554704443835 \\
\hline 109 & $\mathrm{H}$ & 0.124503075782328 & 0.123647755532530 & 0.292087646548758 \\
\hline 110 & $\mathrm{H}$ & 0.374257724045854 & 0.375381518317816 & 0.289774284587643 \\
\hline 111 & $\mathrm{H}$ & 0.624618482671628 & 0.625742276943590 & 0.289774284587643 \\
\hline 112 & $\mathrm{H}$ & 0.876352244467469 & 0.875496924217671 & 0.292087646548758 \\
\hline 113 & $\mathrm{H}$ & 0.128999721031902 & 0.374903448650172 & 0.357870438814899 \\
\hline 114 & $\mathrm{H}$ & 0.374065282853855 & 0.128277385353101 & 0.355565252639728 \\
\hline
\end{tabular}




$\begin{array}{lllll}115 & \mathrm{H} & 0.625096551349827 & 0.871000279957542 & 0.357870438814899 \\ 116 & \mathrm{H} & 0.871722615636343 & 0.625934717146144 & 0.355565252639728 \\ 117 & \mathrm{H} & 0.115502302149636 & 0.626914486047311 & 0.423159945637301 \\ 118 & \mathrm{H} & 0.373085514942133 & 0.884497698839808 & 0.423159945637301 \\ 119 & \mathrm{H} & 0.624499811437694 & 0.110736445626777 & 0.424128996682853 \\ 120 & \mathrm{H} & 0.889263555362667 & 0.375500189551750 & 0.424128996682853 \\ 121 & \mathrm{H} & 0.117920651971253 & 0.882079348028746 & 0.489832498518779 \\ 122 & \mathrm{H} & 0.864239049920456 & 0.135760950079543 & 0.487537803224618 \\ 123 & \mathrm{H} & 0.642021544884961 & 0.357978748188926 & 0.463322312683331 \\ 124 & \mathrm{Ru} & 0.524907136872393 & 0.475092923088034 & 0.461392400424408 \\ 125 & \mathrm{Ru} & 0.312963764387177 & 0.478860437367153 & 0.495927198722633 \\ 126 & \mathrm{Ru} & 0.461804913091633 & 0.304665603449130 & 0.509270665671032 \\ 127 & \mathrm{Ru} & 0.521139622593267 & 0.687036235612822 & 0.495927198722633 \\ 128 & \mathrm{Ru} & 0.695334396550869 & 0.538195146868794 & 0.509270665671032\end{array}$

Table S12: Fractional atomic coordinates of the final state of the NH formation on $\mathrm{Ru} / \mathrm{Ca}_{2} \mathrm{NH}$. Lattice parameters are: $\mathrm{a}=\mathrm{b}=10.1067 \AA, \mathrm{c}=39.0701 \AA, \alpha=\beta=\gamma=90$.

$\begin{array}{ccccc}\text { No. } & \text { Atom } & X & Y & Z \\ 1 & \mathrm{Ca} & 0.108115437024963 & 0.391911555158699 & 0.030150181941693 \\ 2 & \mathrm{Ca} & 0.391911555158699 & 0.108115437024963 & 0.030150181941693 \\ 3 & \mathrm{Ca} & 0.608088474821514 & 0.891884570890603 & 0.030150181941693 \\ 4 & \mathrm{Ca} & 0.891884570890603 & 0.608088474821514 & 0.030150181941693 \\ 5 & \mathrm{Ca} & 0.139734689832295 & 0.860265266137361 & 0.034964078332301 \\ 6 & \mathrm{Ca} & 0.360208036288476 & 0.639791993691737 & 0.034944222405848 \\ 7 & \mathrm{Ca} & 0.639791993691737 & 0.360208036288476 & 0.034944222405848 \\ 8 & \mathrm{Ca} & 0.860265266137361 & 0.139734689832295 & 0.034964078332301 \\ 9 & \mathrm{Ca} & 0.136610523539424 & 0.636753359259927 & 0.094502413941925 \\ 10 & \mathrm{Ca} & 0.363246670819222 & 0.863389431440786 & 0.094502413941925 \\ 11 & \mathrm{Ca} & 0.636753359259927 & 0.136610523539424 & 0.094502413941925 \\ 12 & \mathrm{Ca} & 0.863389431440786 & 0.363246670819222 & 0.094502413941925 \\ 13 & \mathrm{Ca} & 0.113566263137904 & 0.113566263137904 & 0.098992774323385 \\ 14 & \mathrm{Ca} & 0.386583350682940 & 0.386583350682940 & 0.098910376787060 \\ 15 & \mathrm{Ca} & 0.613416679297273 & 0.613416679297273 & 0.098910376787060 \\ 16 & \mathrm{Ca} & 0.886433744876612 & 0.886433744876612 & 0.098992774323385 \\ 17 & \mathrm{Ca} & 0.112350651944332 & 0.887649356070177 & 0.157983219441412\end{array}$




\begin{tabular}{|c|c|c|c|c|}
\hline & $\mathrm{a}$ & & & \\
\hline & & 612619904321846 & & 0.15792605272789 \\
\hline & & 887649356070177 & .112350651944332 & 0.15798321944141 \\
\hline & & & & 0 \\
\hline & $\mathrm{Ca}$ & .363227812474697 & .137486222954997 & 0.16443605090304 \\
\hline & $\mathrm{Ca}$ & 09 & & 304 \\
\hline & $\mathrm{Ca}$ & 5213 & 17505509 & 0.1644360 \\
\hline & $\mathrm{Ca}$ & & & \\
\hline & $\mathrm{Ca}$ & & & \\
\hline & & & & 0.2 \\
\hline & $\mathrm{Ca}$ & & & \\
\hline & & & & \\
\hline & $\mathrm{Ca}$ & & 56478640 & 0.2290 \\
\hline & $\mathrm{Ca}$ & 843092 & & \\
\hline & $\mathrm{Ca}$ & 888671662134385 & & 0.228 \\
\hline & $\mathrm{Ca}$ & & & \\
\hline & & & & \\
\hline & & & & \\
\hline & & & & \\
\hline & $\mathrm{Ca}$ & 0.133102026977049 & 0.866897928992607 & \\
\hline & & & & 845 \\
\hline & $\mathrm{Ca}$ & & & \\
\hline & & & & 539 \\
\hline & & 0.132302006785366 & & 0.352 \\
\hline & $\mathrm{Ca}$ & & & \\
\hline & & & & \\
\hline & $\mathrm{Ca}$ & & & 220 \\
\hline & $\mathrm{Ca}$ & & 0.116060456335795 & 0.36365979858918 \\
\hline & $\mathrm{Ca}$ & 0.390599728185606 & 0.389193960828293 & 0.3570 \\
\hline & & 0.610806070141364 & 0.609400302784052 & 0.35702836435023 \\
\hline & & & & 0.36365979858918 \\
\hline & $\mathrm{Ca}$ & 0.109610594602379 & 0.890389413412130 & 0.42256158635883 \\
\hline & & & 0.630533772154990 & \\
\hline 51 & $\mathrm{Ca}$ & 0.635116640639701 & 0.364883389439455 & 0.41143239936783 \\
\hline & $\mathrm{Ca}$ & 0.889800349413576 & 0.110199658600933 & 0.42289082498421 \\
\hline
\end{tabular}




\begin{tabular}{|c|c|c|c|c|}
\hline & $\mathrm{Ca}$ & & & \\
\hline & $\mathrm{Ca}$ & 0.381500821965559 & 0.158276842841615 & (1272501602012 \\
\hline & $\mathrm{Ca}$ & .619126520186831 & 0.835930132778194 & 042042053001100 \\
\hline & $\mathrm{Ca}$ & 3113128040 & 0.618499208014654 & 1070-016 \\
\hline & $\mathrm{Ca}$ & 168597997137553 & 0.168893758468328 & 0.48915760658110 \\
\hline & $\mathrm{Ca}$ & & & \\
\hline & $\mathrm{Ca}$ & 0.096949711660663 & 0.638903033380422 & 0.4904330029037 \\
\hline & $\mathrm{Ca}$ & & & \\
\hline & $\mathrm{Ca}$ & 9404 & & 0.4 \\
\hline & $\mathrm{Ca}$ & 910760437855557 & 0.365683003 & \\
\hline & $\mathrm{N}$ & 121479989738574 & 3502800040 & \\
\hline & $\mathrm{N}$ & 0.378446497199959 & 0.878520010261425 & 0.0348035098624 \\
\hline & $\mathrm{N}$ & 621553502800040 & 574 & \\
\hline & $\mathrm{N}$ & 878520010261425 & & \\
\hline 67 & $\mathrm{~N}$ & 124247063514481 & 2936485518 & \\
\hline & $\mathrm{N}$ & 375495294464329 & 0.624504705535670 & 0.09 \\
\hline 69 & $\mathrm{~N}$ & 624504705535670 & 0.375495294464329 & 0.09628 \\
\hline & $\mathrm{N}$ & & & \\
\hline & $\mathrm{N}$ & .125408497299822 & 0.125408497299822 & 0.1614086735365 \\
\hline & $\mathrm{N}$ & 374956399867613 & 0.374956399867613 & \\
\hline & $\mathrm{N}$ & & 386 & \\
\hline & $\mathrm{N}$ & 2700177 & 502700177 & \\
\hline & $\mathrm{N}$ & 126214276495936 & 810 & \\
\hline 76 & $\mathrm{~N}$ & 0.376056091816444 & 0.125763921407184 & \\
\hline 77 & $\mathrm{~N}$ & 624050017253189 & 0.873785723504063 & 0.2262715525262 \\
\hline 78 & $\mathrm{~N}$ & & & \\
\hline 79 & $\mathrm{~N}$ & 023 & 4023 & 0.29 \\
\hline & $\mathrm{N}$ & 371121296265421 & & \\
\hline 81 & $\mathrm{~N}$ & 888 & 100 & \\
\hline & $\mathrm{N}$ & & 0.3706 & \\
\hline 83 & $\mathrm{~N}$ & 916 & 0.877 & \\
\hline 84 & $\mathrm{~N}$ & 0.371430347444821 & 0.628569653544623 & 0.3525458628903 \\
\hline 85 & $\mathrm{~N}$ & 0.629760391425897 & 0.370239609563547 & 0.35216216634 \\
\hline 86 & $\mathrm{~N}$ & 0.877311714728243 & 0.122688285271756 & 0.3598617426345 \\
\hline r & $\mathrm{N}$ & 0.136338960268574 & 0.136268240777610 & 0.4278176262599 \\
\hline
\end{tabular}




\begin{tabular}{|c|c|c|c|c|}
\hline 88 & $\mathrm{~N}$ & 0.395948138153407 & 0.391231476817374 & 0.416913381964249 \\
\hline 89 & $\mathrm{~N}$ & 0.608768523182625 & 0.604051861846592 & 0.416913381964249 \\
\hline 90 & $\mathrm{~N}$ & 0.863731760211834 & 0.863661040720870 & 0.427817626259937 \\
\hline 91 & $\mathrm{~N}$ & .134275398427284 & 0.402306322708952 & 0.490764787042842 \\
\hline 92 & $\mathrm{~N}$ & 0.399081411146390 & 0.128237701073068 & 0.487881485895385 \\
\hline 93 & $\mathrm{~N}$ & 0.597693677291047 & 0.865724602562160 & 0.490764787042842 \\
\hline 94 & $\mathrm{~N}$ & 0.871762299916376 & 0.600918588853609 & 0.487881485895385 \\
\hline 95 & $\mathrm{~N}$ & 0.342021545234601 & 0.657978484844555 & 0.506721619909711 \\
\hline 96 & $\mathrm{~N}$ & 0.635188296238922 & 0.364811733840234 & 0.517827952193428 \\
\hline 97 & $\mathrm{H}$ & 0.133723336371751 & 0.133723336371751 & 0.032621928917692 \\
\hline 98 & $\mathrm{H}$ & 0.366273334736391 & 0.366273334736391 & 0.032601939743728 \\
\hline 99 & $\mathrm{H}$ & 0.633726666253053 & 0.633726666253053 & 0.032601939743728 \\
\hline 100 & $\mathrm{H}$ & 0.866276664617693 & 0.866276664617693 & 0.032621928917692 \\
\hline 101 & $\mathrm{H}$ & 0.124749278064634 & 0.625437115934694 & 0.161574204132399 \\
\hline 102 & $\mathrm{H}$ & 0.374562884065305 & 0.875250721935366 & 0.161574204132399 \\
\hline 103 & $\mathrm{H}$ & 0.625437115934694 & 0.124749278064634 & 0.161574204132399 \\
\hline 104 & $\mathrm{H}$ & 0.875250721935366 & 0.374562884065305 & 0.161574204132399 \\
\hline 105 & $\mathrm{H}$ & 0.122752569518603 & 0.877247430481396 & 0.226970756225476 \\
\hline 106 & $\mathrm{H}$ & 0.374375908783875 & 0.625624092205569 & 0.226238906731154 \\
\hline 107 & $\mathrm{H}$ & 0.625831584159797 & 0.374168416829640 & 0.226171198619376 \\
\hline 108 & $\mathrm{H}$ & 0.877461371525889 & 0.122538628474110 & 0.226955264986905 \\
\hline 109 & $\mathrm{H}$ & 0.125017955738364 & 0.124572989833403 & 0.292340461905119 \\
\hline 110 & $\mathrm{H}$ & 0.375361222469706 & 0.374714892222279 & 0.290192841186815 \\
\hline 111 & $\mathrm{H}$ & 0.625285108767165 & 0.624638778519738 & 0.290192841186815 \\
\hline 112 & $\mathrm{H}$ & 0.875427010166596 & 0.874982044261635 & 0.292340461905119 \\
\hline 113 & $\mathrm{H}$ & 0.129576660073304 & 0.375198748630282 & 0.356812856793020 \\
\hline 114 & $\mathrm{H}$ & 0.376594763496335 & 0.127134574405680 & 0.357425790566821 \\
\hline 115 & $\mathrm{H}$ & 0.624801251369717 & 0.870423340916140 & 0.356812856793020 \\
\hline 116 & $\mathrm{H}$ & 0.872865426583764 & 0.623405236503664 & 0.357425790566821 \\
\hline 117 & $\mathrm{H}$ & 0.121298942893055 & 0.628698654161986 & 0.424105775452034 \\
\hline 118 & $\mathrm{H}$ & 0.371301346827458 & 0.878701058096389 & 0.424105775452034 \\
\hline 119 & $\mathrm{H}$ & 0.629215689102764 & 0.115766391558750 & 0.423585264937649 \\
\hline 120 & $\mathrm{H}$ & 0.884233609430694 & 0.370784311886680 & 0.423585264937649 \\
\hline 121 & $\mathrm{H}$ & 0.123492069180457 & 0.876507930819542 & 0.486817360151306 \\
\hline 122 & $\mathrm{H}$ & 0.871074086074664 & 0.128925913925336 & 0.487717719561132 \\
\hline
\end{tabular}




$\begin{array}{lllll}123 & \mathrm{H} & 0.635343141730189 & 0.364656888348967 & 0.544231491824525 \\ 124 & \mathrm{Ru} & 0.503409839205289 & 0.496590220755130 & 0.459520527966574 \\ 125 & \mathrm{Ru} & 0.311222614156008 & 0.475000396626114 & 0.501678040646751 \\ 126 & \mathrm{Ru} & 0.461615143021411 & 0.299405526011817 & 0.499372375560625 \\ 127 & \mathrm{Ru} & 0.524999663334305 & 0.688777385843991 & 0.501678040646751 \\ 128 & \mathrm{Ru} & 0.700594473988182 & 0.538384916939016 & 0.499372375560625\end{array}$

Table S13: Fractional atomic coordinates of the initial state of the $\mathrm{NH}_{2}$ formation on $\mathrm{Ru} / \mathrm{Ca}_{2} \mathrm{NH}$. Lattice parameters are: $\mathrm{a}=\mathrm{b}=10.1067 \AA, \mathrm{c}=39.0701 \AA, \alpha=\beta=\gamma=90^{\circ}$.

$\begin{array}{ccccc}\text { No. } & \text { Atom } & X & Y & Z \\ 1 & \mathrm{Ca} & 0.108115437024963 & 0.391911555158699 & 0.030150181941693 \\ 2 & \mathrm{Ca} & 0.391911555158699 & 0.108115437024963 & 0.030150181941693 \\ 3 & \mathrm{Ca} & 0.608088474821514 & 0.891884570890603 & 0.030150181941693 \\ 4 & \mathrm{Ca} & 0.891884570890603 & 0.608088474821514 & 0.030150181941693 \\ 5 & \mathrm{Ca} & 0.139734689832295 & 0.860265266137361 & 0.034964078332301 \\ 6 & \mathrm{Ca} & 0.360208036288476 & 0.639791993691737 & 0.034944222405848 \\ 7 & \mathrm{Ca} & 0.639791993691737 & 0.360208036288476 & 0.034944222405848 \\ 8 & \mathrm{Ca} & 0.860265266137361 & 0.139734689832295 & 0.034964078332301 \\ 9 & \mathrm{Ca} & 0.136610523539424 & 0.636753359259927 & 0.094502413941925 \\ 10 & \mathrm{Ca} & 0.363246670819222 & 0.863389431440786 & 0.094502413941925 \\ 11 & \mathrm{Ca} & 0.636753359259927 & 0.136610523539424 & 0.094502413941925 \\ 12 & \mathrm{Ca} & 0.863389431440786 & 0.363246670819222 & 0.094502413941925 \\ 13 & \mathrm{Ca} & 0.113566263137904 & 0.113566263137904 & 0.098992774323385 \\ 14 & \mathrm{Ca} & 0.386583350682940 & 0.386583350682940 & 0.098910376787060 \\ 15 & \mathrm{Ca} & 0.613416679297273 & 0.613416679297273 & 0.098910376787060 \\ 16 & \mathrm{Ca} & 0.886433744876612 & 0.886433744876612 & 0.098992774323385 \\ 17 & \mathrm{Ca} & 0.112350651944332 & 0.887649356070177 & 0.157983219441412 \\ 18 & \mathrm{Ca} & 0.387380126647812 & 0.612619904321846 & 0.157926052727894 \\ 19 & \mathrm{Ca} & 0.612619904321846 & 0.387380126647812 & 0.157926052727894 \\ 20 & \mathrm{Ca} & 0.887649356070177 & 0.112350651944332 & 0.157983219441412 \\ 21 & \mathrm{Ca} & 0.137486222954997 & 0.363227812474697 & 0.164436050903042 \\ 22 & \mathrm{Ca} & 0.363227812474697 & 0.137486222954997 & 0.164436050903042 \\ 23 & \mathrm{Ca} & 0.636772217505509 & 0.862513732025213 & 0.164436050903042 \\ 24 & \mathrm{Ca} & 0.862513732025213 & 0.636772217505509 & 0.164436050903042 \\ 25 & \mathrm{Ca} & 0.137102555934618 & 0.137098563966553 & 0.223038725349532 \\ & & & \\ & & \end{array}$




\begin{tabular}{|c|c|c|c|c|}
\hline & $\mathrm{Ca}$ & & & \\
\hline & $\mathrm{Ca}$ & 0.636243178163105 & 0.636282769961468 & 020281020162788 \\
\hline & $\mathrm{Ca}$ & 862901392003102 & 0.862897400035038 & 02720207252405 \\
\hline & $\mathrm{Ca}$ & 111755185471551 & 614979900315184 & 2201157207070 \\
\hline & & 385020130753417 & & 07070 \\
\hline & $\mathrm{Ca}$ & & & \\
\hline & $\mathrm{Ca}$ & 888807452468292 & & 0.228942 \\
\hline & $\mathrm{Ca}$ & & & \\
\hline & $\mathrm{Ca}$ & 387542319370162 & 0.114539522 & 0.2876609175105 \\
\hline & $\mathrm{Ca}$ & & & \\
\hline & & & & \\
\hline & $\mathrm{Ca}$ & & & \\
\hline & & 87522 & 691 & 020 \\
\hline & $\mathrm{Ca}$ & & 100 & \\
\hline & & & & \\
\hline & & 131810002658220 & & \\
\hline & $\mathrm{Ca}$ & & & \\
\hline & & 139 & 514 & \\
\hline & $\mathrm{Ca}$ & 0.868487716520697 & 0.361400560535074 & \\
\hline & & & & \\
\hline & $\mathrm{Ca}$ & 388333841918104 & 0.388683824732098 & $02566 \Rightarrow$ \\
\hline & & & & \\
\hline & $\mathrm{Ca}$ & 883817585119750 & 731400 & \\
\hline & & 504 & 0.890083 & \\
\hline & & & & \\
\hline & $\mathrm{Ca}$ & & & \\
\hline & $\mathrm{Ca}$ & 888646775792700 & 221809 & 0.4236838939391 \\
\hline & $\mathrm{Ca}$ & .162677458923475 & 0.380135492634373 & 0.429675799 \\
\hline & $\mathrm{Ca}$ & 0.382845035532412 & 0.159597890655497 & 04204073051634 \\
\hline & $\mathrm{Ca}$ & & & \\
\hline & $\mathrm{Ca}$ & 0.840402065314158 & 0.617154994 & 0.4294073051634 \\
\hline & $\mathrm{Ca}$ & 0.169612507009276 & & \\
\hline & $\mathrm{Ca}$ & 0.831701344761725 & 0.830387448960380 & 0.4901601360893 \\
\hline 59 & $\mathrm{Ca}$ & 0.100100985027907 & 0.639314385177392 & 0.491960708435 \\
\hline & $\mathrm{Ca}$ & 0.360685644802814 & 0.899899022986609 & 0.4919607084353 \\
\hline
\end{tabular}




\begin{tabular}{|c|c|c|c|c|}
\hline & $\mathrm{Ca}$ & 635056212581083 & & \\
\hline & $\mathrm{Ca}$ & 0.913355749309630 & 0.364943817399130 & 0.49457058976147 \\
\hline & $\mathrm{N}$ & 121479989738574 & 0.621553502800040 & 0.03480350986244 \\
\hline & & 378446497199959 & 0.878520010 & 0.03 \\
\hline & & 621553502800040 & 0.121479989738574 & 0.03480350986244 \\
\hline & $\mathrm{N}$ & 261425 & 46497199959 & 6244 \\
\hline & $\mathrm{N}$ & 124247063514481 & 0.875752936485518 & 0.09637348319447 \\
\hline & & 5294464329 & & 0.096284 \\
\hline & $\mathrm{N}$ & .624504705535670 & & 0.09628474375754 \\
\hline & & 875752936485518 & 7063514481 & 0.09637348319447 \\
\hline & $\mathrm{N}$ & 0.125408497299822 & & 0.16140867353657 \\
\hline & & 374956399867613 & 0.374956399867613 & 0.16116066805636 \\
\hline 73 & $\mathrm{~N}$ & 2386 & 0.6250 & 5626 \\
\hline & $\mathrm{N}$ & 0.874591502700177 & 0.874591502700177 & 0.1614 \\
\hline & $\mathrm{N}$ & 126158065101421 & 0.376190556068444 & 0.22624390020479 \\
\hline & $\mathrm{N}$ & & & 3373 \\
\hline 77 & $\mathrm{~N}$ & 0.623809443931556 & 0.873841934898578 & 0.22624390020479 \\
\hline 78 & $\mathrm{~N}$ & 874198006704965 & 0.624114138 & 3373 \\
\hline 79 & $\mathrm{~N}$ & & 28683298350 & 0.29 \\
\hline 80 & $\mathrm{~N}$ & 691094 & 0.879 & \\
\hline 81 & $\mathrm{~N}$ & .629571604941631 & & 0.290 \\
\hline 82 & $\mathrm{~N}$ & 0.879724582068583 & 0.370428396047813 & 0.290901871316222 \\
\hline 8 & $\mathrm{~N}$ & 44224060999 & 75939000 & 2178 \\
\hline & $\mathrm{N}$ & & & \\
\hline 85 & $\mathrm{~N}$ & & & 09345 \\
\hline & $\mathrm{N}$ & & & \\
\hline 87 & $\mathrm{~N}$ & 53 & 27 & \\
\hline & $\mathrm{N}$ & & & \\
\hline 89 & $\mathrm{~N}$ & 0.610452483078347 & 0.607158294442456 & 0.416756695100602 \\
\hline 90 & $\mathrm{~N}$ & 0.863769782885818 & 0.863946772685181 & 0.42890358814360 \\
\hline 91 & $\mathrm{~N}$ & 0.137625825846456 & 0.402876666583154 & 0.49130298011703 \\
\hline 92 & $\mathrm{~N}$ & 0.399004760424318 & 0.124586344284672 & 0.490672653722420 \\
\hline 93 & $\mathrm{~N}$ & 6846 & 42989 & 0298011703 \\
\hline 94 & $\mathrm{~N}$ & 0.875413656704772 & 0.600995239575681 & 0.490672653722420 \\
\hline 05 & $\mathrm{~N}$ & 0.344845690020505 & 0.655154340058651 & 0.50969308485645 \\
\hline
\end{tabular}




\begin{tabular}{|c|c|c|c|c|}
\hline 96 & $\mathrm{~N}$ & 0.634618408228533 & 0.365381621850623 & 0.522396346770214 \\
\hline 97 & $\mathrm{H}$ & 0.133723336371751 & 0.133723336371751 & 0.032621928917692 \\
\hline 98 & $\mathrm{H}$ & 0.366273334736391 & 0.366273334736391 & 0.032601939743728 \\
\hline 99 & $\mathrm{H}$ & 0.633726666253053 & 0.633726666253053 & 0.032601939743728 \\
\hline 100 & $\mathrm{H}$ & 0.866276664617693 & 0.866276664617693 & 0.032621928917692 \\
\hline 101 & $\mathrm{H}$ & 0.124749278064634 & 0.625437115934694 & 0.161574204132399 \\
\hline 102 & $\mathrm{H}$ & 0.374562884065305 & 0.875250721935366 & 0.161574204132399 \\
\hline 103 & $\mathrm{H}$ & 0.625437115934694 & 0.124749278064634 & 0.161574204132399 \\
\hline 104 & $\mathrm{H}$ & 0.875250721935366 & 0.374562884065305 & 0.161574204132399 \\
\hline 105 & $\mathrm{H}$ & 0.122674473865487 & 0.877325526134512 & 0.226972046008732 \\
\hline 106 & $\mathrm{H}$ & 0.374252381405192 & 0.625747619584252 & 0.226289071873019 \\
\hline 107 & $\mathrm{H}$ & 0.625628874915776 & 0.374371126073668 & 0.226026853715254 \\
\hline 108 & $\mathrm{H}$ & 0.877455335369660 & 0.122544664630346 & 0.226859032384545 \\
\hline 109 & $\mathrm{H}$ & 0.124480043324744 & 0.124570562383731 & 0.292229665602555 \\
\hline 110 & $\mathrm{H}$ & 0.374028318376581 & 0.374731564825630 & 0.290088943081400 \\
\hline 111 & $\mathrm{H}$ & 0.625268436163814 & 0.625971682612863 & 0.290088943081400 \\
\hline 112 & $\mathrm{H}$ & 0.875429437616269 & 0.875519956675255 & 0.292229665602555 \\
\hline 113 & $\mathrm{H}$ & 0.128296403711196 & 0.375762999444695 & 0.357064320938242 \\
\hline 114 & $\mathrm{H}$ & 0.375717035703310 & 0.127590505189743 & 0.356941129034339 \\
\hline 115 & $\mathrm{H}$ & 0.624237000555304 & 0.871703597278248 & 0.357064320938242 \\
\hline 116 & $\mathrm{H}$ & 0.872409495799701 & 0.624282964296689 & 0.356941129034339 \\
\hline 117 & $\mathrm{H}$ & 0.122617785588325 & 0.629314283388815 & 0.425212721913901 \\
\hline 118 & $\mathrm{H}$ & 0.370685717600636 & 0.877382215401119 & 0.425212721913901 \\
\hline 119 & $\mathrm{H}$ & 0.627741034595679 & 0.117152435066458 & 0.424061031992394 \\
\hline 120 & $\mathrm{H}$ & 0.882847565922986 & 0.372258966393765 & 0.424061031992394 \\
\hline 121 & $\mathrm{H}$ & 0.124827396888832 & 0.875172603111167 & 0.487582433160753 \\
\hline 122 & $\mathrm{H}$ & 0.871149595949908 & 0.128850404050091 & 0.488926002694931 \\
\hline 123 & $\mathrm{H}$ & 0.632944561527985 & 0.367055468551171 & 0.548683208547991 \\
\hline 124 & $\mathrm{H}$ & 0.615419835643962 & 0.384580224316458 & 0.466596058779011 \\
\hline 125 & $\mathrm{Ru}$ & 0.502455621475924 & 0.497544438484496 & 0.461518125016617 \\
\hline 126 & $\mathrm{Ru}$ & 0.314413380048634 & 0.472065313561039 & 0.502570313093684 \\
\hline 127 & $\mathrm{Ru}$ & 0.462673163555017 & 0.294543837892405 & 0.502486602208371 \\
\hline 128 & $\mathrm{Ru}$ & 0.527934746399381 & 0.685586619951365 & 0.502570313093684 \\
\hline 129 & $\mathrm{Ru}$ & 0.705456162107594 & 0.537326896405410 & 0.502486602208371 \\
\hline
\end{tabular}


Table S14: Fractional atomic coordinates of the final state of the $\mathrm{NH}_{2}$ formation on $\mathrm{Ru} / \mathrm{Ca}_{2} \mathrm{NH}$. Lattice parameters are: $\mathrm{a}=\mathrm{b}=10.1067 \AA, \mathrm{c}=39.0701 \AA, \alpha=\beta=\gamma=90$ 。

\begin{tabular}{|c|c|c|c|c|}
\hline 0. & Itom & $X$ & $Y$ & $Z$ \\
\hline 1 & $\mathrm{Ca}$ & 0.108115437024964 & 0.391911555158700 & 0.0301501819416 \\
\hline 2 & $\mathrm{Ca}$ & .391911555158700 & .108115437024964 & 0.03015018194165 \\
\hline & $\mathrm{Ca}$ & 0.608088474821514 & 0.891884570890603 & 0.03015018194169 \\
\hline & $\mathrm{Ca}$ & .891884570890603 & .608088474821514 & 0.0301501819416 \\
\hline & $\mathrm{Ca}$ & 139734689832295 & 0.860265266137361 & 0.03496407833230 \\
\hline & $\mathrm{Ca}$ & .360208036288476 & 0.639791993691738 & 0.03494422240584 \\
\hline & $\mathrm{Ca}$ & 0.639791993691738 & 0.360208036288476 & 0.03494422240584 \\
\hline & $\mathrm{Ca}$ & 0.860265266137361 & 0.139734689832295 & 0.034 \\
\hline 9 & $\mathrm{Ca}$ & 0.136610523539424 & 0.636753359259927 & 0.09450241394192 \\
\hline 10 & $\mathrm{Ca}$ & 246670819223 & 0.863389431440786 & 0.09450241394192 \\
\hline 1 & $\mathrm{Ca}$ & 0.636753359259927 & 0.136610523539424 & 0.09450241394192 \\
\hline & $\mathrm{Ca}$ & 0.863389431440786 & 0.363246670819223 & 0.0945024 \\
\hline 15 & $\mathrm{Ca}$ & 7905 & & \\
\hline 14 & $\mathrm{Ca}$ & 0.386583350682940 & & 0.09891037678706 \\
\hline & $\mathrm{Ca}$ & 0.61341667 & & 0.098 \\
\hline & $\mathrm{Ca}$ & 0.886433744876612 & & \\
\hline 17 & $\mathrm{Ca}$ & 0.112350651944332 & 0.887649356070177 & \\
\hline 18 & $\mathrm{Ca}$ & 0.38738012 & 19904321846 & 0.1579 \\
\hline 19 & $\mathrm{Ca}$ & 0.612619904321846 & 0.387380126647813 & \\
\hline & $\mathrm{Ca}$ & 177 & & 0 . \\
\hline 21 & $\mathrm{Ca}$ & 0.137486222954998 & 0.363227812474697 & 0.16443605090304 \\
\hline 22 & $\mathrm{Ca}$ & 74697 & 54998 & 90304 \\
\hline רט & $\mathrm{Ca}$ & 0.636772217505509 & & \\
\hline 24 & $\mathrm{Ca}$ & 0.862513732025214 & 0.636772217505509 & 0.1 \\
\hline & $\mathrm{Ca}$ & & & \\
\hline 26 & $\mathrm{Ca}$ & 0.363443394893203 & 0.36353554302 & 0.22 \\
\hline 20 & $\mathrm{Ca}$ & 0.636464486957408 & 0.636556635087011 & 0.22300211190135 \\
\hline 28 & $\mathrm{Ca}$ & 0.863019935401923 & 0.862891681762378 & \\
\hline 0 & $\mathrm{Ca}$ & 0.112901260434974 & 0.615026069274023 & 0.22934429731557 \\
\hline 30 & $\mathrm{Ca}$ & 4579 & 7579536 & 0.22 \\
\hline 31 & $\mathrm{Ca}$ & 0.614018830750857 & 0.111057988470925 & 0.22887523327381 \\
\hline & $\mathrm{Ca}$ & 0.888942019543585 & 0.385981200317744 & 0.2288752332738 \\
\hline
\end{tabular}




\begin{tabular}{|c|c|c|c|c|}
\hline & $\mathrm{Ca}$ & & & \\
\hline & $\mathrm{Ca}$ & .386960190522004 & & \\
\hline & $\mathrm{Ca}$ & 612133302131028 & 0.885337491148022 & 02875080345184 \\
\hline & & 070660 & 203 & \\
\hline & $\mathrm{Ca}$ & 134332000239652 & 0.865667955730004 & 0.2957636788408 \\
\hline & $\mathrm{Ca}$ & 3552 & 6654 & \\
\hline & $\mathrm{Ca}$ & 639836051184616 & 0.360163978795597 & 02014644440060 \\
\hline & $\mathrm{Ca}$ & 6614 & & \\
\hline & $\mathrm{Ca}$ & & & \\
\hline & $\mathrm{Ca}$ & .360528138845616 & 0.865083813120279 & (1) \\
\hline & $\mathrm{Ca}$ & .635379927677164 & 0.134783231888875 & \\
\hline & $\mathrm{Ca}$ & 865216723091336 & 0.364620102303050 & \\
\hline & $\mathrm{Ca}$ & .114208902897651 & 078 & \\
\hline & $\mathrm{Ca}$ & 538269592 & 0.388767711718195 & 0.3554 \\
\hline & $\mathrm{Ca}$ & 611232319251464 & 0.613487492701054 & 0.3554696 \\
\hline & & & & \\
\hline & $\mathrm{Ca}$ & .112434549680046 & 0.887565458334464 & \\
\hline & $\mathrm{Ca}$ & 413 & 239690744 & \\
\hline & & & & \\
\hline 52 & $\mathrm{Ca}$ & 0.887818584451252 & & \\
\hline & & .153547792176298 & 0.380372760102219 & \\
\hline 54 & $\mathrm{Ca}$ & 0.368860657210604 & 0.152573330591800 & 0.42862 \\
\hline & $\mathrm{Ca}$ & 77995 & 359 & \\
\hline & $\mathrm{Ca}$ & & 2769610 & \\
\hline & $\mathrm{Ca}$ & 957 & & \\
\hline & $\mathrm{Ca}$ & & 0.849453503356699 & \\
\hline & $\mathrm{Ca}$ & 803 & & \\
\hline & & & & \\
\hline 61 & $\mathrm{Ca}$ & 0.615603204708876 & 0.110451742246852 & \\
\hline & $\mathrm{Ca}$ & .889548265767658 & 0.384396825271338 & 04014772660121 \\
\hline 63 & $\mathrm{~N}$ & 121479989738575 & 0.621553502800040 & \\
\hline 64 & $\mathrm{~N}$ & 0.378446497199960 & 0.878520010261425 & 0.0348035098624 \\
\hline 65 & $\mathrm{~N}$ & 0.621553502800040 & 0.121479989738575 & 0.03480350986 \\
\hline 66 & $\mathrm{~N}$ & 0.878520010261425 & 0.378446497199960 & 0.034803509862 \\
\hline & $\mathrm{N}$ & 0.124247063514481 & 0.875752936485519 & 0.096373483194 \\
\hline
\end{tabular}




\begin{tabular}{|c|c|c|c|c|}
\hline 68 & $\mathrm{~N}$ & 0.375495294464329 & 0.624504705535670 & 0.096284743757543 \\
\hline 69 & $\mathrm{~N}$ & 0.624504705535670 & 0.375495294464329 & 0.096284743757543 \\
\hline 70 & $\mathrm{~N}$ & 0.875752936485519 & 0.124247063514481 & 0.096373483194476 \\
\hline 71 & $\mathrm{~N}$ & 0.125408497299823 & 0.125408497299823 & 0.161408673536570 \\
\hline 72 & $\mathrm{~N}$ & 0.374956399867614 & 0.374956399867614 & 0.161160668056368 \\
\hline 73 & $\mathrm{~N}$ & 0.625043600132386 & 0.625043600132386 & 0.161160668056368 \\
\hline 74 & $\mathrm{~N}$ & 0.874591502700177 & 0.874591502700177 & 0.161408673536570 \\
\hline 75 & $\mathrm{~N}$ & 0.126287189215432 & 0.375883961724369 & 0.225926650795238 \\
\hline 76 & $\mathrm{~N}$ & 0.375227950768569 & 0.125369940033920 & 0.226014743238473 \\
\hline 77 & $\mathrm{~N}$ & 0.624116038275631 & 0.873712810784568 & 0.225926650795238 \\
\hline 78 & $\mathrm{~N}$ & 0.874630059966080 & 0.624772049231431 & 0.226014743238473 \\
\hline 79 & $\mathrm{~N}$ & 0.122490331669752 & 0.627871184870671 & 0.291240816330713 \\
\hline 80 & $\mathrm{~N}$ & 0.372128816118774 & 0.877509668330248 & 0.291240816330713 \\
\hline 81 & $\mathrm{~N}$ & 0.628602971312432 & 0.120764120097775 & 0.290373037750562 \\
\hline 82 & $\mathrm{~N}$ & 0.879235879902225 & 0.371397029677013 & 0.290373037750562 \\
\hline 83 & $\mathrm{~N}$ & 0.119934316189607 & 0.880065683810386 & 0.359593026438013 \\
\hline 84 & $\mathrm{~N}$ & 0.371494872995001 & 0.628505127994444 & 0.354772594484878 \\
\hline 85 & $\mathrm{~N}$ & 0.627375942270127 & 0.372624058719317 & 0.350417061757518 \\
\hline 86 & $\mathrm{~N}$ & 0.875120368526339 & 0.124879631473661 & 0.359256178334682 \\
\hline 87 & $\mathrm{~N}$ & 0.128802069660843 & 0.135446609473384 & 0.428227387800312 \\
\hline 88 & $\mathrm{~N}$ & 0.385732761811823 & 0.371147736906245 & 0.414117933081613 \\
\hline 89 & $\mathrm{~N}$ & 0.628852263093755 & 0.614267238188177 & 0.414117933081613 \\
\hline 90 & $\mathrm{~N}$ & 0.864553391516068 & 0.871197931328609 & 0.428227387800312 \\
\hline 91 & $\mathrm{~N}$ & 0.119187943263860 & 0.416241606363037 & 0.485100091425963 \\
\hline 92 & $\mathrm{~N}$ & 0.381231395474074 & 0.136170112211559 & 0.496210077387076 \\
\hline 93 & $\mathrm{~N}$ & 0.583758393636963 & 0.880812057725592 & 0.485100091425963 \\
\hline 94 & $\mathrm{~N}$ & 0.863829888777886 & 0.618768604525918 & 0.496210077387076 \\
\hline 95 & $\mathrm{~N}$ & 0.342083974924371 & 0.657916055154786 & 0.493586661641153 \\
\hline 96 & $\mathrm{~N}$ & 0.562556194373947 & 0.437443835705210 & 0.543420785763814 \\
\hline 97 & $\mathrm{H}$ & 0.133723336371752 & 0.133723336371752 & 0.032621928917692 \\
\hline 98 & $\mathrm{H}$ & 0.366273334736391 & 0.366273334736391 & 0.032601939743728 \\
\hline 99 & $\mathrm{H}$ & 0.633726666253054 & 0.633726666253054 & 0.032601939743728 \\
\hline 100 & $\mathrm{H}$ & 0.866276664617693 & 0.866276664617693 & 0.032621928917692 \\
\hline 101 & $\mathrm{H}$ & 0.124749278064634 & 0.625437115934695 & 0.161574204132400 \\
\hline 102 & $\mathrm{H}$ & 0.374562884065305 & 0.875250721935366 & 0.161574204132400 \\
\hline
\end{tabular}




$\begin{array}{lllll}103 & \mathrm{H} & 0.625437115934695 & 0.124749278064634 & 0.161574204132400 \\ 104 & \mathrm{H} & 0.875250721935366 & 0.374562884065305 & 0.161574204132400 \\ 105 & \mathrm{H} & 0.123587678601517 & 0.876412321398483 & 0.226367869490993 \\ 106 & \mathrm{H} & 0.374408486592046 & 0.625591514397406 & 0.225836519010137 \\ 107 & \mathrm{H} & 0.625938353789920 & 0.374061647199532 & 0.225305970565906 \\ 108 & \mathrm{H} & 0.877112775035492 & 0.122887224964508 & 0.226214815159672 \\ 109 & \mathrm{H} & 0.123688228272762 & 0.124188741271780 & 0.291826636404046 \\ 110 & \mathrm{H} & 0.374208127199758 & 0.375737816812865 & 0.289371883788739 \\ 111 & \mathrm{H} & 0.624262184176580 & 0.625791873789687 & 0.289371883788739 \\ 112 & \mathrm{H} & 0.875811258728220 & 0.876311771727238 & 0.291826636404046 \\ 113 & \mathrm{H} & 0.124263504699023 & 0.374418522729023 & 0.356571720991099 \\ 114 & \mathrm{H} & 0.370304984855458 & 0.127110790673996 & 0.354035532601863 \\ 115 & \mathrm{H} & 0.625581477270977 & 0.875736496290422 & 0.356571720991099 \\ 116 & \mathrm{H} & 0.872889210315456 & 0.629695015144542 & 0.354035532601863 \\ 117 & \mathrm{H} & 0.107487339481395 & 0.624986373599128 & 0.417635673385462 \\ 118 & \mathrm{H} & 0.375013627390317 & 0.892512661508050 & 0.417635673385462 \\ 119 & \mathrm{H} & 0.619604894750779 & 0.117384095069028 & 0.423056337571033 \\ 120 & \mathrm{H} & 0.882615905920417 & 0.380395106238666 & 0.423056337571033 \\ 121 & \mathrm{H} & 0.115355180113909 & 0.884644819886091 & 0.496948233089832 \\ 122 & \mathrm{H} & 0.857198522799585 & 0.142801477200415 & 0.487000340628853 \\ 123 & \mathrm{H} & 0.503514768367900 & 0.496485261711257 & 0.558365744094679 \\ 124 & \mathrm{H} & 0.617723120945627 & 0.382276909133530 & 0.560280948285431 \\ 125 & \mathrm{Ru} & 0.546391310302610 & 0.453608749657811 & 0.474070716880512 \\ 126 & \mathrm{Ru} & 0.295187715906991 & 0.478758769937095 & 0.493692434819231 \\ 127 & \mathrm{Ru} & 0.436334090227469 & 0.306646678181295 & 0.512007028750311 \\ 128 & \mathrm{Ru} & 0.521241290023326 & 0.704812284093009 & 0.493692434819231 \\ 129 & \mathrm{Ru} & 0.693353321818705 & 0.563665969732952 & 0.512007028750311\end{array}$

Table S15: Fractional atomic coordinates of the initial state of the $\mathrm{NH}_{3}$ formation on $\mathrm{Ru} / \mathrm{Ca}_{2} \mathrm{NH}$. Lattice parameters are: $\mathrm{a}=\mathrm{b}=10.1067 \AA, \mathrm{c}=39.0701 \AA, \alpha=\beta=\gamma=90$ 。
No. Atom
$X$ $Y$
$Z$
$\begin{array}{lll}1 & \mathrm{Ca} & 0.108115437024964 \\ 2 & \mathrm{Ca} & 0.391911555158700 \\ 3 & \mathrm{Ca} & 0.608088474821514 \\ 4 & \mathrm{Ca} & 0.891884570890603\end{array}$
0.391911555158700
0.030150181941693
0.108115437024964
0.030150181941693
0.891884570890603
0.030150181941693
0.608088474821514
0.030150181941693 


$\begin{array}{lllll}5 & \mathrm{Ca} & 0.139734689832295 & 0.860265266137361 & 0.034964078332301 \\ 6 & \mathrm{Ca} & 0.360208036288476 & 0.639791993691738 & 0.034944222405848 \\ 7 & \mathrm{Ca} & 0.639791993691738 & 0.360208036288476 & 0.034944222405848 \\ 8 & \mathrm{Ca} & 0.860265266137361 & 0.139734689832295 & 0.034964078332301 \\ 9 & \mathrm{Ca} & 0.136610523539424 & 0.636753359259927 & 0.094502413941925 \\ 10 & \mathrm{Ca} & 0.363246670819223 & 0.863389431440786 & 0.094502413941925 \\ 11 & \mathrm{Ca} & 0.636753359259927 & 0.136610523539424 & 0.094502413941925 \\ 12 & \mathrm{Ca} & 0.863389431440786 & 0.363246670819223 & 0.094502413941925 \\ 13 & \mathrm{Ca} & 0.113566263137905 & 0.113566263137905 & 0.098992774323385 \\ 14 & \mathrm{Ca} & 0.386583350682940 & 0.386583350682940 & 0.098910376787060 \\ 15 & \mathrm{Ca} & 0.613416679297273 & 0.613416679297273 & 0.098910376787060 \\ 16 & \mathrm{Ca} & 0.886433744876612 & 0.886433744876612 & 0.098992774323385 \\ 17 & \mathrm{Ca} & 0.112350651944332 & 0.887649356070177 & 0.157983219441412 \\ 18 & \mathrm{Ca} & 0.387380126647813 & 0.612619904321846 & 0.157926052727895 \\ 19 & \mathrm{Ca} & 0.612619904321846 & 0.387380126647813 & 0.157926052727895 \\ 20 & \mathrm{Ca} & 0.887649356070177 & 0.112350651944332 & 0.157983219441412 \\ 21 & \mathrm{Ca} & 0.137486222954998 & 0.363227812474697 & 0.164436050903042 \\ 22 & \mathrm{Ca} & 0.363227812474697 & 0.137486222954998 & 0.164436050903042 \\ 23 & \mathrm{Ca} & 0.636772217505509 & 0.862513732025214 & 0.164436050903042 \\ 24 & \mathrm{Ca} & 0.862513732025214 & 0.636772217505509 & 0.164436050903042 \\ 25 & \mathrm{Ca} & 0.136951103994868 & 0.137137610207255 & 0.223222868539303 \\ 26 & \mathrm{Ca} & 0.363230921224734 & 0.363654989415224 & 0.223052798505933 \\ 27 & \mathrm{Ca} & 0.636345040564990 & 0.636769108755480 & 0.223052798505933 \\ 28 & \mathrm{Ca} & 0.862862345762401 & 0.863048851974789 & 0.223222868539303 \\ 29 & \mathrm{Ca} & 0.112782025936355 & 0.615325814914385 & 0.229454729224564 \\ 30 & \mathrm{Ca} & 0.384674216154217 & 0.887217982078155 & 0.229454729224564 \\ 31 & \mathrm{Ca} & 0.613830319182902 & 0.111041424354660 & 0.228908587208515 \\ 32 & \mathrm{Ca} & 0.888958583659850 & 0.386169711885699 & 0.228908587208515 \\ 33 & \mathrm{Ca} & 0.114548252693119 & 0.388153440336708 & 0.287693351829816 \\ \mathrm{Ca} & 0.386691904091073 & 0.113147872991668 & 0.287948944831683 \\ 37 & \mathrm{Ca} & 0.611846589643498 & 0.885451755321391 & 0.287693351829816 \\ 39 & 0.886852135022849 & 0.613308125889134 & 0.287948944831683 \\ 30.133843399371038 & 0.866156556598618 & 0.296005426983690 \\ 30 & 0.639596885958809 & 0.360403144021404 & 0.291286185702397\end{array}$




\begin{tabular}{|c|c|c|c|c|}
\hline & $\mathrm{Ca}$ & & & \\
\hline & & 0.134634735540338 & 0.640133645163019 & 0.35434590884027 \\
\hline & $\mathrm{Ca}$ & 359866384817188 & 0.865365219439873 & 0.35434590884027 \\
\hline & & 634649038268066 & .134911451456851 & 0.35133002757029 \\
\hline & $\mathrm{Ca}$ & 865088503523360 & & 0.35133002 \\
\hline & $\mathrm{Ca}$ & & & \\
\hline & & 385972527434404 & 0.389133882161040 & 0.35554680456535 \\
\hline & $\mathrm{Ca}$ & & & \\
\hline & & 883669722937810 & 0.886848050995759 & 0.36286041091226 \\
\hline & $\mathrm{Ca}$ & & & \\
\hline & & & & 0.4195 \\
\hline & $\mathrm{Ca}$ & & & \\
\hline & & & & $0-0=010$ \\
\hline & $\mathrm{Ca}$ & & & \\
\hline & $\mathrm{Ca}$ & & & \\
\hline & & & & \\
\hline & $\mathrm{Ca}$ & & & \\
\hline & & 7532 & & 0.488 \\
\hline & $\mathrm{Ca}$ & 0.819744331784904 & 0.850271068822124 & 0.48843 \\
\hline & & & & \\
\hline 60 & $\mathrm{Ca}$ & 354505679338452 & 0.9175827 & 0.503067 \\
\hline & & & & \\
\hline & $\mathrm{Ca}$ & 887887167566753 & 0.385005346856914 & \\
\hline & $\mathrm{N}$ & & & \\
\hline & & 99960 & & \\
\hline 65 & $\mathrm{~N}$ & 00040 & & \\
\hline 66 & $\mathrm{~N}$ & 878520010261425 & 0.378446497199960 & 0.03480350986244 \\
\hline 67 & $\mathrm{~N}$ & 0.124247063514481 & 0.875752936485519 & 0.0963734 \\
\hline 68 & $\mathrm{~N}$ & 0.375495294464329 & 0.624504705535670 & 0.0962847437575 \\
\hline 69 & $\mathrm{~N}$ & & & \\
\hline 70 & $\mathrm{~N}$ & 0.875752936485519 & 0.124247063514481 & 0.09637348319447 \\
\hline 71 & $\mathrm{~N}$ & & & \\
\hline 72 & $\mathrm{~N}$ & 0.374956399867614 & 0.374956399867614 & 0.1611606680563 \\
\hline 73 & $\mathrm{~N}$ & 0.625043600132386 & 0.625043600132386 & 0.1611606680563 \\
\hline 74 & $\mathrm{~N}$ & 0.874591502700177 & 0.874591502700177 & 0.1614086735365 \\
\hline
\end{tabular}




\begin{tabular}{|c|c|c|c|c|}
\hline & $\mathrm{N}$ & 0.126255887361538 & 0.376128457779060 & \\
\hline & & 0.374942818178816 & 0.125452700915069 & .22599742907966 \\
\hline & & 0.623871542220940 & 0.873744112638462 & 0.22587910005739 \\
\hline & & .874547299084931 & .625057181821184 & 022599742907966 \\
\hline & & 0.122212134848546 & 0.628236601406194 & 0.29137555047352 \\
\hline & $\mathrm{N}$ & & & 52 \\
\hline & $\mathrm{N}$ & 0.628288781944342 & 0.120665538796601 & 0.29030211023967 \\
\hline & $\mathrm{N}$ & & & \\
\hline & $\mathrm{N}$ & & 0.880625380620060 & 0.35972449718654 \\
\hline & $\mathrm{N}$ & & & \\
\hline & & 0.626980518540030 & 0.373019482449415 & 0.34990391637551 \\
\hline & $\mathrm{N}$ & & & 0.359 \\
\hline & & 0.127914115640912 & 0.136401592890785 & 0.42837180894746 \\
\hline & $\mathrm{N}$ & 0.387223218067660 & 0.370723877576900 & 0.41429143104409 \\
\hline & $\mathrm{N}$ & 23100 & 2340 & \\
\hline & $\mathrm{N}$ & 0.863598408098666 & 0.872085885348532 & 0.4283718089474 \\
\hline & $\mathrm{N}$ & 0.121274263291809 & 0.416839388762817 & 0.4852582 \\
\hline & $\mathrm{N}$ & 0.380837401961344 & 0.136945 & 0.4970793 \\
\hline & $\mathrm{N}$ & 0.583160611237183 & 0.878725737697636 & 0.4852582626717 \\
\hline & & & & 0.4 \\
\hline & $\mathrm{N}$ & 0.342683295391119 & 0.657316734688038 & 0.49761733877647 \\
\hline & $\mathrm{N}$ & 530 & & 0.54 \\
\hline & $\mathrm{H}$ & 0.133723336371752 & 0.133723336371752 & 0.0326219289176 \\
\hline & $\mathrm{H}$ & 0.366273334736391 & 0.366273334736391 & 0.0326 \\
\hline & $\mathrm{H}$ & 0.633726666253054 & 0.633726666253054 & 003260 \\
\hline & $\mathrm{H}$ & 0.866276664617693 & 0.866 & 0.032 \\
\hline & $\mathrm{H}$ & 0.124749278064634 & 0.625437115934695 & 0.16157420413240 \\
\hline & $\mathrm{H}$ & 0.374562884065305 & 0.875250721935366 & 0.16157420413240 \\
\hline & $\mathrm{H}$ & 0.625437115934695 & 0.124749278064634 & 0.16157420413240 \\
\hline & $\mathrm{H}$ & 0.875250721935366 & 0.374562884065305 & 0.16157420413240 \\
\hline & $\mathrm{H}$ & 0.123384991915671 & 0.876615008084329 & 0.22625877486997 \\
\hline & $\mathrm{H}$ & 0.374067673822616 & 0.625932327166829 & 0.22578 \\
\hline & $\mathrm{H}$ & 0.625762395637992 & 0.374237605351453 & 0.2251655191548 \\
\hline & $\mathrm{H}$ & 0.876796722053925 & 0.123203277946075 & 0.2260766409562 \\
\hline & $\mathrm{H}$ & 0.123141689419704 & 0.124447605314089 & 0.2917602562984 \\
\hline
\end{tabular}




$\begin{array}{lllll}110 & \mathrm{H} & 0.373654498719311 & 0.376048035782830 & 0.289306681392993 \\ 111 & \mathrm{H} & 0.623951965206615 & 0.626345502280031 & 0.289306681392993 \\ 112 & \mathrm{H} & 0.875552394685911 & 0.876858310570405 & 0.291760256298424 \\ 113 & \mathrm{H} & 0.125014648153190 & 0.374881142096370 & 0.356420531221147 \\ 114 & \mathrm{H} & 0.369518750008112 & 0.127135464663041 & 0.354358455040270 \\ 115 & \mathrm{H} & 0.625118857903630 & 0.874985352836255 & 0.356420531221147 \\ 116 & \mathrm{H} & 0.872864536326404 & 0.630481249991888 & 0.354358455040270 \\ 117 & \mathrm{H} & 0.109985865537453 & 0.628225464193014 & 0.418692556426137 \\ 118 & \mathrm{H} & 0.371774536796430 & 0.890014135451999 & 0.418692556426137 \\ 119 & \mathrm{H} & 0.612847680986519 & 0.109099415900786 & 0.421750764251122 \\ 120 & \mathrm{H} & 0.890900585088659 & 0.387152320002926 & 0.421750764251122 \\ 121 & \mathrm{H} & 0.118519151907194 & 0.881480848092806 & 0.495215452693976 \\ 122 & \mathrm{H} & 0.860283733630965 & 0.139716266369035 & 0.488778058708768 \\ 123 & \mathrm{H} & 0.508714211028646 & 0.491285819050510 & 0.559167755971025 \\ 124 & \mathrm{H} & 0.622478935131703 & 0.377521094947454 & 0.561304734945836 \\ 125 & \mathrm{H} & 0.673424072278884 & 0.326575987681544 & 0.465210158370645 \\ 126 & \mathrm{Ru} & 0.550244158178700 & 0.449755901781728 & 0.473455439064189 \\ 127 & \mathrm{Ru} & 0.299128762134607 & 0.478383929804842 & 0.495168969118701 \\ 128 & \mathrm{Ru} & 0.435347459439683 & 0.305775383134829 & 0.513422542015128 \\ 129 & \mathrm{Ru} & 0.521616130155578 & 0.700871237865393 & 0.495168969118701 \\ 130 & \mathrm{Ru} & 0.694224616865171 & 0.564652600520738 & 0.513422542015128\end{array}$

Table S16: Fractional atomic coordinates of the final state of the $\mathrm{NH}_{3}$ formation on $\mathrm{Ru} / \mathrm{Ca}_{2} \mathrm{NH}$. Lattice parameters are: $\mathrm{a}=\mathrm{b}=10.1067 \AA, \mathrm{c}=39.0701 \AA, \alpha=\beta=\gamma=90^{\circ}$. No. Atom

$$
X
$$

$\begin{array}{ccc}1 & \mathrm{Ca} & 0.108115437024964 \\ 2 & \mathrm{Ca} & 0.391911555158700 \\ 3 & \mathrm{Ca} & 0.608088474821514 \\ 4 & \mathrm{Ca} & 0.891884570890603 \\ 5 & \mathrm{Ca} & 0.139734689832295 \\ 6 & \mathrm{Ca} & 0.360208036288476 \\ 7 & \mathrm{Ca} & 0.639791993691738 \\ 8 & \mathrm{Ca} & 0.860265266137361 \\ 9 & \mathrm{Ca} & 0.136610523539424 \\ 10 & \mathrm{Ca} & 0.363246670819223\end{array}$

Y

0.391911555158700

0.108115437024964

0.891884570890603

0.608088474821514

0.860265266137361

0.639791993691738

0.360208036288476

0.139734689832295

0.636753359259927

0.863389431440786

\section{$Z$}

0.030150181941693

0.030150181941693

0.030150181941693

0.030150181941693

0.034964078332301

0.034944222405848

0.034944222405848

0.034964078332301

0.094502413941925

0.094502413941925 


$\begin{array}{lllll}11 & \mathrm{Ca} & 0.636753359259927 & 0.136610523539424 & 0.094502413941925 \\ 12 & \mathrm{Ca} & 0.863389431440786 & 0.363246670819223 & 0.094502413941925 \\ 13 & \mathrm{Ca} & 0.113566263137905 & 0.113566263137905 & 0.098992774323385 \\ 14 & \mathrm{Ca} & 0.386583350682940 & 0.386583350682940 & 0.098910376787060 \\ 15 & \mathrm{Ca} & 0.613416679297273 & 0.613416679297273 & 0.098910376787060 \\ 16 & \mathrm{Ca} & 0.886433744876612 & 0.886433744876612 & 0.098992774323385 \\ 17 & \mathrm{Ca} & 0.112350651944332 & 0.887649356070177 & 0.157983219441412 \\ 18 & \mathrm{Ca} & 0.387380126647813 & 0.612619904321846 & 0.157926052727895 \\ 19 & \mathrm{Ca} & 0.612619904321846 & 0.387380126647813 & 0.157926052727895 \\ 20 & \mathrm{Ca} & 0.887649356070177 & 0.112350651944332 & 0.157983219441412 \\ 21 & \mathrm{Ca} & 0.137486222954998 & 0.363227812474697 & 0.164436050903042 \\ 22 & \mathrm{Ca} & 0.363227812474697 & 0.137486222954998 & 0.164436050903042 \\ 23 & \mathrm{Ca} & 0.636772217505509 & 0.862513732025214 & 0.164436050903042 \\ 24 & \mathrm{Ca} & 0.862513732025214 & 0.636772217505509 & 0.164436050903042 \\ 25 & \mathrm{Ca} & 0.136671347154845 & 0.136865616451090 & 0.223180476916710 \\ 26 & \mathrm{Ca} & 0.362982959536758 & 0.363235427072404 & 0.223128850924361 \\ 27 & \mathrm{Ca} & 0.636179535371944 & 0.636545329264329 & 0.223086655428482 \\ 28 & \mathrm{Ca} & 0.862580857392011 & 0.862835894835342 & 0.223234231349899 \\ 29 & \mathrm{Ca} & 0.112154819956778 & 0.614426190478774 & 0.229298862287877 \\ 30 & \mathrm{Ca} & 0.384904612386855 & 0.886974011260939 & 0.229209446787479 \\ 31 & \mathrm{Ca} & 0.613404990111795 & 0.111664873348708 & 0.229047611526958 \\ 32 & \mathrm{Ca} & 0.887551378008460 & 0.385778384609139 & 0.229037512911702 \\ 33 & \mathrm{Ca} & 0.112882024842420 & 0.387480850055866 & 0.287724160194735 \\ 34 & \mathrm{Ca} & 0.386227649821663 & 0.112330581815492 & 0.287867784088711 \\ 35 & \mathrm{Ca} & 0.611353011580903 & 0.885912004867805 & 0.287721206016066 \\ 36 & \mathrm{Ca} & 0.886230511091483 & 0.612567964337785 & 0.287880021541483 \\ 37 & \mathrm{Ca} & 0.134199122702633 & 0.864672476253141 & 0.295411275708389 \\ 38 & \mathrm{Ca} & 0.359120445089658 & 0.639145930060245 & 0.293664563786351 \\ 40 & \mathrm{Ca} & 0.638527375429710 & 0.360215411717313 & 0.292472939676863 \\ & \mathrm{Ca} & 0.135520571146657 & 0.637580062649263 & 0.353549842927983 \\ 43 & 0.360817825368130 & 0.862431787024349 & 0.353770592102947 \\ 4.63413299033108696 & 0.114782729232537 & 0.361189158208973\end{array}$




\begin{tabular}{|c|c|c|c|c|}
\hline & $\mathrm{Ca}$ & & & \\
\hline & & .608440467476946 & .612165170481298 & 0.35502252496415 \\
\hline & $\mathrm{Ca}$ & 883897030644690 & 0.884908092456151 & 0.36154698067576 \\
\hline & $\mathrm{Ca}$ & & & 125 \\
\hline & $\mathrm{Ca}$ & 396964051438935 & 0.599929407248595 & 0.42143378 \\
\hline & $\mathrm{Ca}$ & & & \\
\hline & $\mathrm{Ca}$ & 885798408194439 & & 0.41998265678985 \\
\hline & $\mathrm{Ca}$ & & & \\
\hline & & 367767271932237 & 0.146882901457865 & 0.42661389792805 \\
\hline & $\mathrm{Ca}$ & & & \\
\hline & & & & 0.426 \\
\hline & $\mathrm{Ca}$ & & & \\
\hline & & 244705251 & 53084 & 0.485 \\
\hline & $\mathrm{Ca}$ & & & \\
\hline & $\mathrm{Ca}$ & & & \\
\hline & & & & \\
\hline & $\mathrm{Ca}$ & & & \\
\hline & $\mathrm{N}$ & 121479989738575 & 800040 & 0.0348 \\
\hline & $\mathrm{N}$ & 0.378446497199960 & 0.878520010261425 & 0.03480350986244 \\
\hline & & & & \\
\hline 66 & $\mathrm{~N}$ & 878520010261425 & 0.378446497199960 & 0.03480350986244 \\
\hline & $\mathrm{N}$ & & & 9447 \\
\hline & & 0.375495294464329 & 0.624504705535670 & 0.09628 \\
\hline & $\mathrm{N}$ & & 0.375 & 0.09 \\
\hline & & & & \\
\hline 71 & $\mathrm{~N}$ & & & $080 / 35305 / 4$ \\
\hline 72 & $\mathrm{~N}$ & 0.374956399867614 & 0.374956399867614 & 0.16116066805636 \\
\hline 73 & $\mathrm{~N}$ & 0.625043600132386 & 0.625043600132386 & 0.16116066805636 \\
\hline 74 & $\mathrm{~N}$ & 0.874591502700177 & 0.874591502700177 & 0.16140867353657 \\
\hline 75 & $\mathrm{~N}$ & & & \\
\hline 76 & $\mathrm{~N}$ & 0.374636006928874 & 0.125320750761958 & 0.22583998819452 \\
\hline 77 & $\mathrm{~N}$ & 467596 & 35089583085 & $258200 / 418 / 92$ \\
\hline 78 & $\mathrm{~N}$ & 0.874120178503247 & 0.624656324636874 & 0.225926252391332 \\
\hline 79 & $\mathrm{~N}$ & 0.121577491253454 & 0.627050452755810 & 0.29103542943666 \\
\hline & $\mathrm{N}$ & 0.371778439339352 & 0.876607813733990 & 0.29077287440856 \\
\hline
\end{tabular}




\begin{tabular}{|c|c|c|c|c|}
\hline 81 & $\mathrm{~N}$ & 0.626728957663367 & 0.121739969731422 & 0.290487941538871 \\
\hline 82 & $\mathrm{~N}$ & 0.877223427760931 & 0.372210537447472 & 0.290533171748045 \\
\hline 83 & $\mathrm{~N}$ & 0.119688813677456 & 0.878203129380772 & 0.358589134841367 \\
\hline 84 & $\mathrm{~N}$ & 0.369412117203559 & 0.626761195800249 & 0.354175068551328 \\
\hline 85 & $\mathrm{~N}$ & 0.627504383562055 & 0.371095263728577 & 0.352280782348515 \\
\hline 86 & $\mathrm{~N}$ & 0.874961679748935 & 0.123379892608234 & 0.358356017046994 \\
\hline 87 & $\mathrm{~N}$ & 0.126307384548760 & 0.132321657883999 & 0.426388296086635 \\
\hline 88 & $\mathrm{~N}$ & 0.377856693819730 & 0.366520249649952 & 0.412504240786262 \\
\hline 89 & $\mathrm{~N}$ & 0.628035637617792 & 0.622413649313074 & 0.412341987615889 \\
\hline 90 & $\mathrm{~N}$ & 0.866362611778897 & 0.871838455220590 & 0.426606450633649 \\
\hline 91 & $\mathrm{~N}$ & 0.127446757400492 & 0.404356299966821 & 0.484826782154194 \\
\hline 92 & $\mathrm{~N}$ & 0.377520765620275 & 0.115047438173320 & 0.492657102702395 \\
\hline 93 & $\mathrm{~N}$ & 0.590021057487029 & 0.871405507950193 & 0.484193842682565 \\
\hline 94 & $\mathrm{~N}$ & 0.876267832249532 & 0.619173030251829 & 0.492074263641513 \\
\hline 95 & $\mathrm{~N}$ & 0.353602160453583 & 0.643283630730565 & 0.492571006234670 \\
\hline 96 & $\mathrm{~N}$ & 0.623010561792988 & 0.532014734787097 & 0.564380332635508 \\
\hline 97 & $\mathrm{H}$ & 0.133723336371752 & 0.133723336371752 & 0.032621928917692 \\
\hline 98 & $\mathrm{H}$ & 0.366273334736391 & 0.366273334736391 & 0.032601939743728 \\
\hline 99 & $\mathrm{H}$ & 0.633726666253054 & 0.633726666253054 & 0.032601939743728 \\
\hline 100 & $\mathrm{H}$ & 0.866276664617693 & 0.866276664617693 & 0.032621928917692 \\
\hline 101 & $\mathrm{H}$ & 0.124749278064634 & 0.625437115934695 & 0.161574204132400 \\
\hline 102 & $\mathrm{H}$ & 0.374562884065305 & 0.875250721935366 & 0.161574204132400 \\
\hline 103 & $\mathrm{H}$ & 0.625437115934695 & 0.124749278064634 & 0.161574204132400 \\
\hline 104 & $\mathrm{H}$ & 0.875250721935366 & 0.374562884065305 & 0.161574204132400 \\
\hline 105 & $\mathrm{H}$ & 0.122849357058435 & 0.876303037368636 & 0.226132911747904 \\
\hline 106 & $\mathrm{H}$ & 0.373654920134221 & 0.625452672557550 & 0.225490172119066 \\
\hline 107 & $\mathrm{H}$ & 0.625406621092679 & 0.374036233327558 & 0.225348808239524 \\
\hline 108 & $\mathrm{H}$ & 0.876294997230026 & 0.123117865600186 & 0.226061765422905 \\
\hline 109 & $\mathrm{H}$ & 0.123170870247847 & 0.124501846521788 & 0.291709973886832 \\
\hline 110 & $\mathrm{H}$ & 0.374146796181627 & 0.375325745356342 & 0.289069463322448 \\
\hline 111 & $\mathrm{H}$ & 0.623765059030504 & 0.624607737845466 & 0.289188372787855 \\
\hline 112 & $\mathrm{H}$ & 0.874255858402435 & 0.874935150536913 & 0.291715441951503 \\
\hline 113 & $\mathrm{H}$ & 0.120108628269620 & 0.375704068622206 & 0.356332213325992 \\
\hline 114 & $\mathrm{H}$ & 0.369409588239151 & 0.124413848258982 & 0.353744291788431 \\
\hline 115 & $\mathrm{H}$ & 0.625205585727763 & 0.876546743291392 & 0.355225874425479 \\
\hline
\end{tabular}




$\begin{array}{lllll}116 & \mathrm{H} & 0.878002865922191 & 0.625601371556478 & 0.355757139477426 \\ 117 & \mathrm{H} & 0.115796307412758 & 0.634607618132399 & 0.425175514141131 \\ 118 & \mathrm{H} & 0.375906369676450 & 0.892757859174885 & 0.416456295121336 \\ 119 & \mathrm{H} & 0.619824072033104 & 0.115140171192110 & 0.423159152162981 \\ 120 & \mathrm{H} & 0.883902327326808 & 0.380996835973583 & 0.423372016827393 \\ 121 & \mathrm{H} & 0.125305219597550 & 0.884430039344245 & 0.491674248274180 \\ 122 & \mathrm{H} & 0.868083574729113 & 0.129892962116287 & 0.486315782267482 \\ 123 & \mathrm{H} & 0.685554191321812 & 0.514727053951603 & 0.584452108978581 \\ 124 & \mathrm{H} & 0.559892897960609 & 0.607934223586376 & 0.570343704890639 \\ 125 & \mathrm{H} & 0.566027499229179 & 0.448415400280112 & 0.559949916069861 \\ 126 & \mathrm{Ru} & 0.567126197601596 & 0.431697904615547 & 0.487474315407482 \\ 127 & \mathrm{Ru} & 0.301128273376023 & 0.463855213612590 & 0.495469489848169 \\ 128 & \mathrm{Ru} & 0.422526539106478 & 0.275863570767690 & 0.511482329102080 \\ 129 & \mathrm{Ru} & 0.531819766882663 & 0.695470437910636 & 0.493785134189892 \\ 130 & \mathrm{Ru} & 0.719789147263604 & 0.572453170395285 & 0.515337565356809\end{array}$

Table S17: Fractional atomic coordinates of the initial state of the $\mathrm{H}$ migration on $\mathrm{Ru} / \mathrm{Ca}_{2} \mathrm{NH}$. Lattice parameters are: $\mathrm{a}=\mathrm{b}=10.1067 \AA, \mathrm{c}=30.4746 \AA, \alpha=\beta=\gamma=90^{\circ}$. No. Atom $X$ $Y$

$\begin{array}{ccccc}1 & \mathrm{Ca} & 0.386888057183512 & 0.386888057183512 & 0.128308384159872 \\ 2 & \mathrm{Ca} & 0.877805028373858 & 0.863986582301237 & 0.290737868022199 \\ 3 & \mathrm{Ca} & 0.643098721800051 & 0.113460203869625 & 0.303227674290101 \\ 4 & \mathrm{Ca} & 0.636888087064782 & 0.136888042143937 & 0.120423211286301 \\ 5 & \mathrm{Ca} & 0.115036757028086 & 0.887451476344237 & 0.206246243657248 \\ 6 & \mathrm{Ca} & 0.129589175719667 & 0.367091017411399 & 0.210380617675085 \\ 7 & \mathrm{Ca} & 0.886888087262669 & 0.613111973093531 & 0.037512678223969 \\ 8 & \mathrm{Ca} & 0.363111943212260 & 0.636888087064782 & 0.045397839940719 \\ 9 & \mathrm{Ca} & 0.136888042143937 & 0.636888087064782 & 0.120423211286301 \\ 10 & \mathrm{Ca} & 0.097964323158110 & 0.560876464046354 & 0.327846797322541 \\ 11 & \mathrm{Ca} & 0.636888087064782 & 0.363111943212260 & 0.045397839940719 \\ 12 & \mathrm{Ca} & 0.613111973093531 & 0.886888087262669 & 0.037512678223969 \\ 13 & \mathrm{Ca} & 0.361728323172194 & 0.139921678194495 & 0.213463326443442 \\ 14 & \mathrm{Ca} & 0.886505592173200 & 0.115321123743286 & 0.205505305848539 \\ 15 & \mathrm{Ca} & 0.375782725353759 & 0.890068929488138 & 0.305008778508185 \\ 16 & \mathrm{Ca} & 0.863111913627832 & 0.363111943212260 & 0.120423211286301\end{array}$




\begin{tabular}{|c|c|c|c|c|}
\hline & $\mathrm{Ca}$ & & & \\
\hline & & 0.610556547411592 & 0.629414643253577 & 0.27894160461043 \\
\hline & $\mathrm{Ca}$ & 113111913232053 & 0.386888057183512 & 0.03751267822396 \\
\hline & $\mathrm{Ca}$ & 136888042143937 & .863111913627832 & 04530783094071 \\
\hline & $\mathrm{Ca}$ & 130879501545856 & 0.144514094573868 & 020102572100550 \\
\hline & $\mathrm{Ca}$ & & & \\
\hline & $\mathrm{Ca}$ & 639357318369740 & 0.865743564729037 & $0.2111332549080^{-}$ \\
\hline & & & & \\
\hline & $\mathrm{Ca}$ & 0.876382923868305 & & 0.2917178948458 \\
\hline & $\mathrm{Ca}$ & & & \\
\hline & $\mathrm{Ca}$ & 2361043772 & & 0.2006963994709 \\
\hline & $\mathrm{Ca}$ & & & \\
\hline & $\mathrm{Ca}$ & 913627832 & 0.136888042143937 & 0.04539783994071 \\
\hline & $\mathrm{Ca}$ & 88057183512 & & \\
\hline & $\mathrm{Ca}$ & & & \\
\hline & & 345765144573200 & & \\
\hline & $\mathrm{N}$ & & & \\
\hline & $\mathrm{N}$ & 375000000197886 & 0.8750000 & 0.04 \\
\hline & $\mathrm{N}$ & 0.875000000395779 & 0.375000000197886 & 0.041 \\
\hline & $\mathrm{N}$ & & & \\
\hline & $\mathrm{N}$ & 376567877117077 & 0.123676474716094 & 0.30154217749277 \\
\hline & $\mathrm{N}$ & & & 7813 \\
\hline & $\mathrm{N}$ & 25000000098943 & 0.875000000395779 & 0.12 \\
\hline & $\mathrm{N}$ & 25000000296836 & 0.12500000009 & 0.0 \\
\hline & $\mathrm{N}$ & & 8413 & \\
\hline 42 & $\mathrm{~N}$ & 18 & & 0.2 \\
\hline & $\mathrm{N}$ & 873948796497237 & 216094393 & 0.29501060611313 \\
\hline 44 & $\mathrm{~N}$ & 0.375000000197886 & 0.625000000296836 & 0.1243658051 \\
\hline & $\mathrm{N}$ & 125000000098943 & 0.625000000296836 & 0.04145526467716 \\
\hline 46 & $\mathrm{~N}$ & & & 0.12 \\
\hline 47 & $\mathrm{~N}$ & 0.629902476396292 & 0.876348721377970 & 0.30039646208917 \\
\hline 48 & $\mathrm{~N}$ & & & \\
\hline 49 & $\mathrm{H}$ & 0.125000000098943 & 0.125000000098943 & 0.0414552646771 \\
\hline 50 & $\mathrm{H}$ & 0.889549037136222 & 0.397294231057244 & 0.2021697597864 \\
\hline & $\mathrm{H}$ & 0.373628360248212 & 0.870659206942354 & 0.2176024378510 \\
\hline
\end{tabular}




$\begin{array}{lllll}52 & \mathrm{H} & 0.639441019484428 & 0.761157536650425 & 0.413464429924343 \\ 53 & \mathrm{H} & 0.875000000395779 & 0.625000000296836 & 0.124365805188318 \\ 54 & \mathrm{H} & 0.625000000296836 & 0.875000000395779 & 0.124365805188318 \\ 55 & \mathrm{H} & 0.733173516681627 & 0.242758489134178 & 0.249296040526096 \\ 56 & \mathrm{H} & 0.118483809514981 & 0.623374903825132 & 0.199581120806266 \\ 57 & \mathrm{H} & 0.875000000395779 & 0.875000000395779 & 0.041455264677161 \\ 58 & \mathrm{H} & 0.375000000197886 & 0.375000000197886 & 0.041455264677161 \\ 59 & \mathrm{H} & 0.375000000197886 & 0.125000000098943 & 0.124365805188318 \\ 60 & \mathrm{H} & 0.891110642850043 & 0.105078287375429 & 0.296102297178015 \\ 61 & \mathrm{H} & 0.588478687250034 & 0.090011298905204 & 0.197474538698451 \\ 62 & \mathrm{H} & 0.127995675509588 & 0.893571940036219 & 0.290749416500366 \\ 63 & \mathrm{H} & 0.125000000098943 & 0.375000000197886 & 0.124365805188318 \\ 64 & \mathrm{H} & 0.625000000296836 & 0.625000000296836 & 0.041455264677161 \\ 65 & \mathrm{Ru} & 0.346960979708212 & 0.415568278823564 & 0.385384344904524 \\ 66 & \mathrm{Ru} & 0.440133628632048 & 0.617828976040256 & 0.356577642042021 \\ 67 & \mathrm{Ru} & 0.432353714569071 & 0.215678987468784 & 0.352349680092656 \\ 68 & \mathrm{Ru} & 0.587212773804535 & 0.422018185802733 & 0.351943731792699 \\ 69 & \mathrm{Ru} & 0.618747948574400 & 0.781994353221698 & 0.355373402316196 \\ 70 & \mathrm{Ru} & 0.793558386291696 & 0.401014011779121 & 0.397311707325070 \\ 71 & \mathrm{Ru} & 0.785971406686548 & 0.587977852454578 & 0.347076924200320 \\ 72 & \mathrm{Ru} & 0.500227847059297 & 0.406116316040101 & 0.442118966228874 \\ 73 & \mathrm{Ru} & 0.614758931330619 & 0.236053787991551 & 0.405527700289821 \\ 74 & \mathrm{Ru} & 0.630525034360575 & 0.578911370339252 & 0.415297845246501\end{array}$

Table S18: Fractional atomic coordinates of the final state of the $\mathrm{H}$ migration on $\mathrm{Ru} / \mathrm{Ca}_{2} \mathrm{NH}$. Lattice parameters are: $\mathrm{a}=\mathrm{b}=10.1067 \AA, \mathrm{c}=30.4746 \AA, \alpha=\beta=\gamma=90$ 。 No. Atom $X$

$\begin{array}{lllll}1 & \mathrm{Ca} & 0.386888057183512 & 0.386888057183512 & 0.128308389246079 \\ 2 & \mathrm{Ca} & 0.878549373557055 & 0.862642046739077 & 0.290983273261680 \\ 3 & \mathrm{Ca} & 0.644056656255637 & 0.114586659456867 & 0.303103595835686 \\ 4 & \mathrm{Ca} & 0.636888087064782 & 0.136888042143937 & 0.120423215880293 \\ 5 & \mathrm{Ca} & 0.114815833586988 & 0.888337850341956 & 0.206389094373705 \\ 6 & \mathrm{Ca} & 0.129623864607897 & 0.368004876087730 & 0.211223020523588 \\ 7 & \mathrm{Ca} & 0.886888087262669 & 0.613111973093531 & 0.037512678388040 \\ 8 & \mathrm{Ca} & 0.363111943212260 & 0.636888087064782 & 0.045397840662631\end{array}$




\begin{tabular}{|c|c|c|c|c|}
\hline 9 & $\mathrm{Ca}$ & 0.136888042143937 & 0.636888087064782 & 0.120423215880293 \\
\hline 10 & $\mathrm{Ca}$ & 0.092643342087416 & 0.558273885548702 & 0.331722893882357 \\
\hline 1 & $\mathrm{Ca}$ & 0.636888087064782 & 0.363111943212260 & 0.045397840662631 \\
\hline 12 & $\mathrm{Ca}$ & 0.613111973093531 & 0.886888087262669 & 0.037512678388040 \\
\hline 13 & $\mathrm{Ca}$ & 0.361649575384209 & 0.140606929934569 & 0.214033642535237 \\
\hline 14 & $\mathrm{Ca}$ & 0.886900998670777 & 0.115349829499969 & 0.205715255714515 \\
\hline 15 & $\mathrm{Ca}$ & 0.373138667533648 & 0.890081246365495 & 0.305365453745226 \\
\hline 16 & $\mathrm{Ca}$ & 0.863111913627832 & 0.363111943212260 & 0.120423215880293 \\
\hline 17 & $\mathrm{Ca}$ & 0.613111973093531 & 0.613111973093531 & 0.128308389246079 \\
\hline 18 & $\mathrm{Ca}$ & 0.610143023627587 & 0.624005324257009 & 0.278175700925035 \\
\hline 19 & $\mathrm{Ca}$ & 0.113111913232053 & 0.386888057183512 & 0.037512678388040 \\
\hline 20 & $\mathrm{Ca}$ & 0.136888042143937 & 0.863111913627832 & 0.045397840662631 \\
\hline 21 & $\mathrm{Ca}$ & 0.131244482769726 & 0.144660260678847 & 0.292515451875048 \\
\hline 22 & $\mathrm{Ca}$ & 0.113111913232053 & 0.113111913232053 & 0.128308389246079 \\
\hline 23 & $\mathrm{Ca}$ & 0.640066421993126 & 0.864948386559055 & 0.211443723126521 \\
\hline 24 & $\mathrm{Ca}$ & 0.615509478891926 & 0.381235619123026 & 0.202260167554227 \\
\hline 25 & $\mathrm{Ca}$ & 0.876000196780667 & 0.361080710818043 & 0.293060148447961 \\
\hline 26 & $\mathrm{Ca}$ & 0.363111943212260 & 0.863111913627832 & 0.120423215880293 \\
\hline 27 & $\mathrm{Ca}$ & 0.379085145725890 & 0.615895177089252 & 0.200069099155522 \\
\hline 28 & $\mathrm{Ca}$ & 0.870562600459499 & 0.637082993926520 & 0.211569689349007 \\
\hline 29 & $\mathrm{Ca}$ & 0.863111913627832 & 0.136888042143937 & 0.045397840662631 \\
\hline 30 & $\mathrm{Ca}$ & 0.386888057183512 & 0.113111913232053 & 0.037512678388040 \\
\hline 31 & $\mathrm{Ca}$ & 0.886888087262669 & 0.886888087262669 & 0.128308389246079 \\
\hline 32 & $\mathrm{Ca}$ & 0.346770237211431 & 0.389427649628358 & 0.285215683335804 \\
\hline 33 & $\mathrm{~N}$ & 0.627979504190079 & 0.629193165012926 & 0.204296713275580 \\
\hline 34 & $\mathrm{~N}$ & 0.375000000197886 & 0.875000000395779 & 0.041455265070937 \\
\hline 35 & $\mathrm{~N}$ & 0.875000000395779 & 0.375000000197886 & 0.041455265070937 \\
\hline 36 & $\mathrm{~N}$ & 0.875000000395779 & 0.125000000098943 & 0.124365806271185 \\
\hline 37 & $\mathrm{~N}$ & 0.376918226628104 & 0.121882769850728 & 0.300469850799767 \\
\hline 38 & $\mathrm{~N}$ & 0.108115570974809 & 0.375805903083026 & 0.288362845387964 \\
\hline 39 & $\mathrm{~N}$ & 0.125000000098943 & 0.875000000395779 & 0.124365806271185 \\
\hline 40 & $\mathrm{~N}$ & 0.625000000296836 & 0.125000000098943 & 0.041455265070937 \\
\hline 41 & $\mathrm{~N}$ & 0.375451095659506 & 0.378394277893373 & 0.208132658089219 \\
\hline 42 & $\mathrm{~N}$ & 0.873800906206894 & 0.873589761723485 & 0.212260107856564 \\
\hline 43 & $\mathrm{~N}$ & 0.874149254316486 & 0.619589348213374 & 0.295540435506319 \\
\hline
\end{tabular}




$\begin{array}{lllll}44 & \mathrm{~N} & 0.375000000197886 & 0.625000000296836 & 0.124365806271185 \\ 45 & \mathrm{~N} & 0.125000000098943 & 0.625000000296836 & 0.041455265070937 \\ 46 & \mathrm{~N} & 0.625000000296836 & 0.375000000197886 & 0.124365806271185 \\ 47 & \mathrm{~N} & 0.631002336857729 & 0.876252167960225 & 0.300212085207178 \\ 48 & \mathrm{~N} & 0.127756643756710 & 0.126542772444146 & 0.209907839241553 \\ 49 & \mathrm{H} & 0.125000000098943 & 0.125000000098943 & 0.041455265070937 \\ 50 & \mathrm{H} & 0.890772968731122 & 0.397806104265659 & 0.202184629836484 \\ 51 & \mathrm{H} & 0.371864582208189 & 0.866186240133312 & 0.219190456767396 \\ 52 & \mathrm{H} & 0.291373892575970 & 0.685795065762153 & 0.331539122636803 \\ 53 & \mathrm{H} & 0.875000000395779 & 0.625000000296836 & 0.124365806271185 \\ 54 & \mathrm{H} & 0.625000000296836 & 0.875000000395779 & 0.124365806271185 \\ 55 & \mathrm{H} & 0.735148233497737 & 0.242608135132723 & 0.249115068419763 \\ 56 & \mathrm{H} & 0.120300606429784 & 0.623680395434456 & 0.199882714796727 \\ 57 & \mathrm{H} & 0.875000000395779 & 0.875000000395779 & 0.041455265070937 \\ 58 & \mathrm{H} & 0.375000000197886 & 0.375000000197886 & 0.041455265070937 \\ 59 & \mathrm{H} & 0.375000000197886 & 0.125000000098943 & 0.124365806271185 \\ 60 & \mathrm{H} & 0.891875513424533 & 0.105048838766061 & 0.295952253420816 \\ 61 & \mathrm{H} & 0.587063867013663 & 0.088828619100459 & 0.197187826253650 \\ 62 & \mathrm{H} & 0.127590196754482 & 0.896156745367171 & 0.290001688230053 \\ 63 & \mathrm{H} & 0.125000000098943 & 0.375000000197886 & 0.124365806271185 \\ 64 & \mathrm{H} & 0.625000000296836 & 0.625000000296836 & 0.041455265070937 \\ 65 & \mathrm{Ru} & 0.339207740013403 & 0.406817419044844 & 0.384241006607882 \\ 66 & \mathrm{Ru} & 0.430093909010134 & 0.619809976275492 & 0.356410282054290 \\ 67 & \mathrm{Ru} & 0.432085907567749 & 0.212298290081456 & 0.351622011959110 \\ 68 & \mathrm{Ru} & 0.584398128773739 & 0.422550969511364 & 0.351915617334046 \\ 69 & \mathrm{Ru} & 0.617485923816058 & 0.775292689907608 & 0.352419126009447 \\ 70 & \mathrm{Ru} & 0.788783617674395 & 0.405010250988337 & 0.398432470739558 \\ 71 & \mathrm{Ru} & 0.781026224555086 & 0.590634799503386 & 0.347270124036933 \\ 72 & \mathrm{Ru} & 0.491299893260877 & 0.406249989616550 & 0.440140356141861 \\ & \mathrm{Ru} & 0.613321929548514 & 0.236624354787298 & 0.405229677989500 \\ & \mathrm{Ru} & 0.621672098108255 & 0.578452984443531 & 0.415354386393963\end{array}$




\section{References}

(1) Foppa, L.; Copéret, C.; Comas-vives, A. Increased back-bonding explains stepedge reactivity and particle size effect for $\mathrm{CO}$ activation on $\mathrm{Ru}$ nanoparticles. $J$. Am. Chem. Soc. 2016, 138 (51), 16655-16668.

(2) Nakao, T.; Tada, T.; Kitano, M.; Sasase, M.; Hosono, H. The key indicator for the control of metal particle sizes on supports from first-principles and experimental observation. J. Phys. Chem. C 2016, 120 (38), 21879-21887.

(3) Abe, H.; Niwa, Y.; Kitano, M.; Inoue, Y.; Sasase, M.; Nakao, T.; Tada, T.; Yokoyama, T.; Hara, M.; Hosono, H. Anchoring bond between Ru and N atoms of $\mathrm{Ru} / \mathrm{Ca}_{2} \mathrm{NH}$ catalyst: crucial for the high ammonia synthesis activity. $J$. Phys. Chem. C 2017, 121 (38), 20900-20904.

(4) Kitano, M.; Inoue, Y.; Ishikawa, H.; Yamagata, K.; Nakao, T.; Tada, T.; Matsuishi, S.; Yokoyama, T.; Hara, M.; Hosono, H. Essential role of hydride ion in ruthenium-based ammonia synthesis catalysts. Chem. Sci. 2016, 7, 4036-4043.

(5) M. W. Chase, NIST-JANAF Thermochemical Tables, Washington, DC: American Chemical Society; New York: American Institute of Physics for the National Institute of Standards and Technology, Fourth Edition, 1998. 